\title{
Probing the backbone topology of DNA: synthesis and properties of 7',5'-bicyclo-DNA
}

Damien Evéquoz and Christian J. Leumann*[a]

Department of Chemistry and Biochemistry

University of Bern

Freiestrasse 3, CH-3012 Bern, Switzerland

E-mail: christian.leumann@dcb.unibe.ch

Supporting information for this article is given via a link at the end of the document.

Abstract: We describe the synthesis and pairing properties of the novel DNA analogue 7',5'-bicyclo(bc)-DNA. In this analogue, the point of attachment of the connecting phosphodiester group is switched from the 3'- to the 7'-position of the underlying bicyclic sugar unit and is thus in a topological position that is inaccessible in natural DNA. The corresponding phosphoramidite building blocks carrying all natural nucleobases were synthesized and incorporated into oligonucleotides. From Tm-experiments of duplexes with complementary DNA and RNA we find that single modifications are generally well tolerated with some variability as to the nature of the nucleobase. Fully modified oligonucleotides show low affinity for RNA and DNA complements. However, they form antiparallel homo-duplexes with similar thermal stability as DNA. CD spectra of homo-duplexes show distinct changes in the helix conformation compared to natural DNA. A conformational analysis at the ab initio level of the mononucleosides revealed two minimal energy structures which primarily deviate in the conformation of the cyclopentane ring. Molecular dynamics simulation of a 7',5'-bc-DNA homo-duplex revealed a right-handed structure with a smaller helical rise and a significantly wider minor groove compared to DNA. Interestingly, this duplex is characterized by an atypical, alternating 6'endo/6'-exo conformational pattern of consecutive nucleotides which seems to be responsible for the poor binding to natural nucleic acids.

\section{Introduction}

The molecular storage and retrieval system for genetic information in all forms of life on earth is DNA and RNA. The question on why nature chose this particular molecular structure as the genetic material has been a matter of debate since the structure and function of the nucleic acids became known. ${ }^{[1]}$ One way to approach this question experimentally is to design alternative nucleic acid structures and test their chemical properties with respect to criteria deemed to be important for function. The generally accepted minimal requirements for function comprise the ability for complementary base-pair formation, for self-replication, for sequence evolution and for performing basic cellular metabolism. Along these lines a whole set of hexose-, pentose- and tetrose-derived nucleic acid alternatives such as hexopyranosyl-, ${ }^{[2]}$ 
pentopyranosyl[-[3] or pentofuranosyl-RNAs ${ }^{[4]}$ have been investigated. Their characteristics as potential prebiotic genetic material has been reviewed by A. Eschenmoser, who pioneered the field in the late eighties. ${ }^{[5]}$ Besides this, other minimal nucleic acid modifications such as the glycol nucleic acids (GNA) ${ }^{[6]}$ and the peptide nucleic acids (PNA) ${ }^{[7]}$ have been investigated in this context.

A significant area of synthetic biology is concerned with the design of artificial, DNA-like genetic systems. A basic requirement to achieve this goal is the development of synthetic genetic polymers, often referred to as xeno-nucleic acids (XNA), that fulfill the function of DNA but that are non-existent in nature. ${ }^{[8]}$ The availability of such systems and their integration into living organisms is expected to equip them with novel properties that are of interest in biotechnology and medicine. From the vast repertoire of nucleic acid modifications that appeared over the last 30 years, a handful of candidates has been scrutinized towards their suitability as an alternative genetic material. To introduce non-natural components into the natural genetic machinery by minimal chemical alteration it has been shown that thymidine in the genome of E. coli strains, lacking thymidylate synthase, can be replaced by 5-chlorouridine in an evolutionary process to a minimum residual thymidine content of less than $2 \% .{ }^{[9]}$ In a different approach it has been reported that unnatural nucleic acids such as hexitol nucleic acids (HNA), ${ }^{[10]}$ cyclohexenyl nucleic acids (CeNA), ${ }^{[11]} 2$ '-fluoroarabino nucleic acids (FANA), ${ }^{[12]}$ arabino nucleic acids (ANA), ${ }^{[13]}$ threose nucleic acids (TNA $)^{[4]}$ and locked nucleic acids (LNA) ${ }^{[14]}$ can be transcribed from and reverse transcribed into DNA by DNA-polymerases obtained from in vitro evolution. ${ }^{[15]}$ Likewise, it has been shown recently that HNA, CeNA, FANA and ANA can be transformed into catalysts (XNAzymes) by SELEX methods utilizing such engineered polymerases, demonstrating that XNAs with alternative backbone structures can fold into active species capable to perform simple chemical steps. ${ }^{[16]}$ An attempt to explore the amenable structural space of known XNAs beyond that occupied by DNA and RNA has recently been performed by comparing known duplex structures in the protein data bank. ${ }^{[17]}$

A seemingly minimal alteration of the backbone structure of RNA and DNA results when changing the point of attachment of the internucleoside phosphate unit from the 3' to the 2' oxygen (2',5'-DNA or 2',5'-RNA). While 2',5'-RNA is a naturally occurring biopolymer, first found in bacteria in the form of 2',5' polyadenylates, ${ }^{[18]} 2^{\prime}, 5^{\prime}-\mathrm{DNA}$ is artificial. The pairing and replication properties of both polymers have been investigated in the past. It was found that 2',5'-RNA binds to complementary RNA but not to DNA. Duplexes with RNA are slightly less stable as compared to pure RNA duplexes and duplexes in the pure 2', $5^{\prime}$-RNA series exist but are even less stable. ${ }^{[19]}$ The same holds true also for 2', 5'-DNA. ${ }^{[20]}$ An NMR structural analysis of a self-complementary 2',5'-DNA duplex reveals a conformation that is close to A-type, showing nucleoside puckers that alternate between S- and N-conformation along the nucleotide chain. ${ }^{[21]}$ In addition, it was shown in primer template extension experiments that stretches of up to four 2', $5^{\prime}$-linked nucleotides on a template can be reverse transcribed into DNA with polymerases or reverse transcriptases even though there is no significant affinity of 2',5'-DNA to natural DNA. ${ }^{[22]}$

In continuing efforts to understand the structure/affinity relationship in nucleic acid analogues we became interested in investigating an artificial nucleic acid system in which the point of attachment of the phosphodiester unit is shifted by the same distance as in 2', $5^{\prime}$-DNA, but in the opposite direction (Figure 1, top left). A simple, conformationally not constrained extension of the backbone by inserting a methylene unit between $\mathrm{C}^{\prime}$ ' and O3' has been explored in the past. These studies revealed that incorporation of such thymidine homologues, either in the $\alpha$ - or $\beta$-configuration, into 
DNA decrease their affinity for natural complements by $1-3^{\circ} \mathrm{C} / \mathrm{mod} .{ }^{[23]}$ In our study we envisaged to use the bicycloDNA (bc-DNA) skeleton that we had designed previously as a conformationally constrained DNA analogue ${ }^{[24]}$ and shift the position of the internucleosidic hydroxyl group from the $3^{\prime}$ to the $7^{\prime}$ position $\left(7^{\prime}, 5^{\prime}\right.$-bc-DNA). Here, we describe the chemical synthesis of 7',5'-bc-DNA nucleosides as well as partially and fully modified oligonucleotides therefrom. By $T_{\mathrm{m}}$ analysis we find that 7',5'-bc-DNA still cross-pairs with natural DNA and RNA, albeit with very low affinity. Self-duplexes, however, exhibit thermal stabilities that are in the same range as that of DNA duplexes. CD-spectroscopy and extensive molecular dynamics simulations show that 7',5'-bc-DNA adopts helix conformations that are distinctly different from that of natural DNA. Thus, this novel DNA analogue carries the structural and base-pairing properties necessary to become an XNA.

\section{Results and Discussion}

Synthesis of nucleosides and phosphoramidite building blocks: The bicyclic scaffolds $\mathbf{7}$ and 10 envisaged for subsequent nucleoside synthesis could be constructed from the previously described intermediate $1^{[25]}($ Scheme 1$)$. The epoxide ring in $\mathbf{1}$ was efficiently opened by LiHMDS mediated intramolecular elimination at $-78^{\circ} \mathrm{C}$, yielding the unsaturated ester 2 in good yields. Subsequent nickel-catalyzed $\mathrm{NaBH}_{4}$ reduction of 2 proceeded stereospecifically from the convex side of the bicyclic core structure, resulting in ester $\mathbf{3}$ as the only identifiable diastereoisomer. The hydroxyl function in $\mathbf{3}$ was then TBDPS protected, giving $\mathbf{4}$ in quantitative yield that was consequently reduced with DIBAL-H at $78^{\circ} \mathrm{C}$, leading to aldehyde $\mathbf{5}$. The acetonide protecting group in $\mathbf{5}$ was then hydrolyzed in aqueous $\mathrm{MeCN}$ with $\ln (\mathrm{OTf})_{3}$ as catalyst, ${ }^{[26]}$ and the resulting bicyclic hemiacetal was converted into the methyl glycoside 6 by simply changing the solvent to $\mathrm{MeOH}$. Compound $\mathbf{6}$ was then acetylated to afford the protected precursor $\mathbf{7}$ that was used for the synthesis of the corresponding purine nucleosides via Vorbrüggen chemistry.

The plan for the synthesis of the pyrimidine nucleosides consisted in the meanwhile well-established application of the $\beta$ stereoselective $\mathrm{NIH}$ induced addition of the nucleobases to a corresponding bicyclic glycal. ${ }^{[27]}$ In our first attempt to introduce the thymine nucleobase, we performed the $\mathrm{N}$-iodosuccinimide (NIS) induced nucleosidation on the direct precursor of glycal 8 with $\mathrm{R}_{1}=$ TMS, that was easily obtained from 6 by treatment with TMSOTf. This approach resulted in the stereoselective formation of the corresponding $\beta$-nucleoside, however, with a significant contamination of $7 \%$ of the $\alpha$-anomer that remained inseparable by standard chromatography techniques. We reasoned that the selectivity could be enhanced by increasing steric bulk at $R_{1}$ and decreasing it at $R_{2}$, as in glycal 10 . This would favor initial $\alpha$-attack of the electrophilic iodine at $\mathrm{C}(3)$ and thus favor the $\beta$-entry of the base. To this end compound 6 was converted to glycal 8 with TMSOTf followed by a short treatment with TBAF to remove the newly introduced TMS group selectively. Intermediate $\mathbf{8}$ was then elaborated into the dimethoxytrityl compound $\mathbf{9}$ which was finally subjected to removal of the TBDPS protecting group with TBAF, giving the desired sugar component 10.

NIS-nucleosidation of the in situ TMS protected glycal 10, followed by radical reduction of the iodide intermediate with $\mathrm{Bu}_{3} \mathrm{SnH}$, yielded the DMTr-protected thymidine derivative 11 in good yield containing only trace amounts $\left(<2 \%\right.$ by ${ }^{1} \mathrm{H}$ NMR) of the a-anomer (Scheme 2). The configuration at the anomeric center in 11 was unambiguously assigned by ${ }^{1} \mathrm{H}$ - 
NMR NOESY experiments (see Supp. Inf.) and by X-ray crystallography (see below). Final phosphitylation with 2 cyanoethyl-N,N,N',N'-tetraisopropyl phosphordiamidite lead to the thymidine phosphoramidite building block 12 . The synthesis of the 5-methylcytidine analogue was achieved by conversion of the base thymine into 5-methylcytosine in nucleoside 11. To this end, nucleoside 11 was TMS protected and converted to the corresponding triazolide by treatment with 1,2,4-triazole and $\mathrm{POCl}_{3}$. Subsequent treatment of this triazolide in a mixture of ammonia and 1,4-dioxane yielded the corresponding 5-methylcytosine nucleoside, which was directly protected with $\mathrm{Bz}_{2} \mathrm{O}$ to give 13 in $88 \%$ yield over three steps. The phosphoramidite 14 was obtained by phosphitylation as described above.

The introduction of the purine nucleobases proved to be more problematic. Classical Vorbrüggen nucleosidation resulted generally in the prevalence of the undesired $\alpha$-nucleosides. In the absence of other stereoselective alternatives, and based on our interest in the $\alpha$-nucleosides in a different context, we nevertheless decided to follow this reaction pathway and converted precursor 7 with either $\mathrm{N}^{6}$-benzoyladenine or 2 -amino-6-chloropurine to the inseparable anomeric mixtures $\mathbf{1 5} \mathbf{a} / \mathbf{b}$ and $\mathbf{2 0} \mathbf{a} / \mathbf{b}$, resp. in $\alpha / \beta$ ratios of $4: 1$ and 7:3 (Scheme 3 ). Separation of anomers was possible only after deacetylation, leading to the pure $\beta$-anomers $\mathbf{1 6}$ and $\mathbf{2 1}$. Here again, the relative configurations at the anomeric centers were unambiguously assigned by ${ }^{1} \mathrm{H}-\mathrm{NMR}$ NOESY experiments (see Supp. Inf.). Likewise, the $\mathrm{N}^{9}$-linkage between the purine bases and the anomeric centers were confirmed by ${ }^{1} \mathrm{H},{ }^{13} \mathrm{C}-\mathrm{HMBC}$ or HSQC correlation experiments (see Supp. Inf.). From here, the adenine building block 19 could be obtained by standard dimethoxytritylation $(\rightarrow 17)$ followed by TBAF mediated cleavage of the silyl protecting group $(\rightarrow \mathbf{1 8})$ and phosphitylation. The synthesis of the guanosine building block required the conversion of the 2-amino-6-chloropurine nucleobase into guanine. This was achieved by treatment of $\mathbf{2 1}$ with 3-hydroxypropionitrile and TBD followed by DMF-protection of the 2-amino group, yielding the protected guanosine derivative 22. Following the same chemical pathway as above, the synthesis of the guanosine building block 25 was achieved by dimethoxytritylation $(\rightarrow 23)$, removal of the silyl protecting group $(\rightarrow$ 24) and phosphitylation.

$X$-ray structure of a 7,'5'-bc-DNA monomer. Despite considerable efforts we were unable to obtain crystals from the free nucleosides or close intermediates along the synthesis pathway outlined in the previous schemes 1-3. We therefore prepared a 7',5'-bc-T analogue, carrying a p-nitrobenzoate group at O7'. Indeed, this compound could be crystallized and its structure solved (Figure 2, for crystallographic and synthetic details see Supp. Inf.). ${ }^{[28]}$ Besides establishing final proof for the relative configuration, we also obtained information on the preferred conformation of the bicyclic sugar scaffold. It clearly emerges that the furanose part exists in a perfect 1 '-exo conformation while the carbocyclic ring occurs in the 6'-endo configuration thus placing the oxy-substituent at $\mathrm{C7}^{\prime}$ in a pseudoaxial and the $5^{\prime} \mathrm{OH}$ group in a pseudoequatorial position. This structure matches exactly one of the two low energy conformers determined by ab initio calculations (see Figure 5a).

Design and synthesis of oligonucleotides: A series of oligonucleotides with single and multiple incorporations of building blocks 12, 14, 19 and 25 as well as fully modified sequences have been synthesized using classical automated phosphoramidite chemistry (for synthetic and analytical details see experimental section). Oligonucleotides with single 
and multiple incorporations were primarily prepared to determine the effect of the modifications on the $T_{\mathrm{m}}$ when complexed with complementary DNA and RNA, as well as to determine the Watson-Crick base pairing selectivity. Fully modified oligonucleotides were synthesized to characterize the pairing behavior not only with natural DNA and RNA, but also to investigate the self-pairing of 7',5'-bc-DNA. The main interest here was to determine whether this novel structural DNA analogue can form an independent base-pairing system.

Pairing properties of modified oligodeoxynucleotides with complementary DNA and RNA: To determine the effect of single and multiple modifications on duplex stability we prepared the modified oligodeoxynucleotides ON1-ON11, represented in Table 1, and measured $T_{\mathrm{m}}$ values for duplexes with complementary DNA and RNA by UV-melting curve analysis.

According to the $\Delta T_{\mathrm{m}} / \mathrm{mod}$ data, the impact of single or double modifications on thermal duplex stability is relatively moderate. Purine modifications (ON6, 7) tend to destabilize duplexes with complementary DNA but show a slight increase in $T_{\mathrm{m}}$ with complementary RNA. Within the pyrimidine series there is, however, more variability. Modified thymidine nucleosides (ON1-3) typically lead to a small depression of $T_{\mathrm{m}}$ with RNA as complement while no clear trend with DNA is observed. Interestingly, both cytosine modifications (ON4, 5, 8-10) significantly stabilize duplexes with complementary DNA while having less effect on the $T_{\mathrm{m}}$ with complementary RNA. As observed previously, the stabilizing effect is more pronounced for the 5-methyl cytosine nucleosides which is in agreement with its known potential to reinforce stacking interactions with neighboring base-pairs.

To determine the base-pairing selectivity we measured $T_{\mathrm{m}} \mathrm{S}$ of $\mathrm{ON} \mathbf{1}$ in complex with complementary DNA having all three possible mismatched bases opposing the site of modification (Supp. Inf., Table S1). The $T_{\mathrm{m} S}$ of the mismatched duplexes are lower by 5.1 to $13^{\circ} \mathrm{C}$ with the G/T wobble pair, as expected, being least destabilizing. These data compare well with the fully natural mismatched series, indicating no significant change in base pairing selectivity of the modification.

Pairing properties of fully modified oligonucleotides with complementary DNA and RNA: A fully modified nonamer consisting of pyrimidine bases only (ON12) as well as 3 dodecamers (ON13-15) containing all four bases were prepared to test their affinity to complementary DNA or RNA in either the antiparallel or parallel orientation. Form the corresponding $T_{\mathrm{m}}$ data (Table 2$)$ it becomes clear that binding is generally weak. Even under high salt $(1 \mathrm{M} \mathrm{NaCl})$ conditions, no melting transition could be observed for ON12 with the antiparallel DNA or RNA complement in the temperature range from $5-80^{\circ} \mathrm{C}$, suggesting that no duplex was formed. However, with the longer dodecamers ON1315, transitions were observed with complementary antiparallel DNA and RNA. The $T_{\mathrm{m}} \mathrm{s}$ of these duplexes are roughly $30^{\circ} \mathrm{C}$ lower as compared to the natural control duplexes, amounting to an average $\Delta T_{\mathrm{m}} / \mathrm{mod}$ of $-2.5^{\circ} \mathrm{C}$. This was not expected from the $T_{\mathrm{m}}$ experiments of single incorporations (Table 1) and highlights the importance of fully modified oligonucleotides when characterizing novel pairing systems. Importantly, no signs of duplex formation were found with the corresponding parallel DNA and RNA complements. This clearly demonstrates that $7^{\prime}, 5^{\prime}-\mathrm{bc}-\mathrm{DNA}$ is still able to communicate with the natural nucleic acids via antiparallel duplex structures, albeit on a substantially lower affinity level. 
Self-pairing of 7',5'-bc-DNA: ON14 has been designed to be the antiparallel and ON15 to be the parallel complement to ON13. With this it was possible to investigate self-pairing of $7^{\prime}, 5^{\prime}-\mathrm{bc}-\mathrm{DNA}$ in both orientations. It turned out that the antiparallel duplex ON13.ON14 showed a classical sigmoidal duplex melting behavior (Figure 3) with a $T_{\mathrm{m}}$ of $54.5^{\circ} \mathrm{C}$, while the constituting single strands on their own did not as evidenced by $T_{\mathrm{m}}$ - and CD- analysis (see Supp. Inf., Figure S5). The $T_{\mathrm{m}}$ is higher by $5.4^{\circ} \mathrm{C}$ compared to the natural duplex of the same sequence. Interestingly the hyperchromicity at $260 \mathrm{~nm}$ of the $7^{\prime}, 5^{\prime}$-bc-DNA duplex amounts to only $20 \%$ of that of the natural duplex. This indicates significantly different stacking arrangement of the bases in both duplex conformations. In the parallel orientation (ON13.ON15) no transition could be found demonstrating the inability of parallel duplex formation in $7^{\prime}, 5^{\prime}$-bc-DNA.

Thermodynamic data of duplex formation: We determined transition enthalpies and entropies for the duplex ON13. ON14 and the corresponding natural duplex by curve fitting to the experimental melting curves in analogy to known methods (Table 3). ${ }^{[29]}$ These data suggest that the natural duplex is enthalpically stabilized while the $7^{\prime}, 5^{\prime}$-bc-DNA duplex is entropically favored. This matches with previous findings in the bc-DNA series and hints to conformational restriction of the $7^{\prime}, 5^{\prime}$-bc-DNA backbone as the origin of the entropic stabilization. The free enthalpies of duplex formation $(\Delta \mathrm{G})$ are in line with the $T_{\mathrm{m}}$ data which qualify them as a measure for thermodynamic duplex stability.

$C D$-spectroscopy: To gain insight into the structural properties of the homo 7',5'-bc-DNA duplex and the hybrid 7',5'-bcDNA/DNA duplex we measured CD-spectra and compared them with that of the corresponding natural DNA duplex (Figure 4). As expected, the natural duplex shows the classical features of a B-DNA duplex. The homo 7',5'-bc-DNA duplex, however, has a CD signature that is significantly different from that of A- or B-type double helices. It is characterized by two minimum ellipticities around 215 and $265 \mathrm{~nm}$ with a maximum at $243 \mathrm{~nm}$. The hybrid duplex carries a signature which is closer to that of the DNA duplex. Compared to B-DNA, the minimum ellipticity at $260 \mathrm{~nm}$ is red shifted by about $15 \mathrm{~nm}$ while the maximum ellipticity at $288 \mathrm{~nm}$ is red-shifted by about $7 \mathrm{~nm}$ and associated with a loss of intensity. The differences in the three CD spectra thus indicate differential arrangements of the bases within the basestack, induced by the change in backbone geometry of each strand. This agrees with the differential hyperchromicities of the two pairing systems in the corresponding UV-melting curves (Figure 3).

Molecular modelling: To gain insight into the possible helix structures formed by $7^{\prime}, 5^{\prime}$-bc-DNA, we performed extensive molecular modeling and dynamics simulation experiments. We first performed a conformational analysis at the HartreeFock ab initio level, on the four 7',5'-bc-nucleosides with the bases A, G, T and ${ }^{5-M e} \mathrm{C}$ (see Supp. Inf., Figure S1-S3). These calculations showed similar energy profiles for each monomer with two distinct minimum energy conformations each. The first conformer (Figure 5a) shows a nearly perfect C1'-exo (S-type) pucker in the furanose subunit and a C6'endo conformation in the carbocyclic ring. In this conformation, the C5' hydroxyl group is in a pseudoequatorial position, orienting the torsion angle $\mathrm{Y}\left(\mathrm{C}^{\prime}\right.$ '-C5') in a "non-natural" antiperiplanar arrangement. The second conformer (Figure 5b) also shows an S-type furanose conformation (C2'-endo sugar pucker). However, the carbocyclic ring is adopting a C6'- 
exo conformation, orienting the C5' hydroxyl group in a pseudoaxial position. Consequently, the $\gamma$ angle is restored to a more "natural" synclinal arrangement. Interestingly, the potential energy surface (PES) of the C6'-exo conformation (Figure 5d) shows a steep convergence toward the minimum, demonstrating structural rigidity, while the C6'-endo conformation seems to allow more flexibility, resulting in a flatter PES (Figure 5c). In terms of stability, the C6'-exo conformer is lower in energy in vacuo (between 0.6 to $1.5 \mathrm{kcal} / \mathrm{mol}$, depending on the nucleobase), while the $\mathrm{C6}^{\prime}$-endo conformer is slightly more stable if solvated in water (between 0.0 to $0.5 \mathrm{kcal} / \mathrm{mol}$ ) (Supp. Inf., Table S2.). Therefore, both structures are likely candidates for defining the conformation of the double helix.

The structures of the previously synthesized 7',5'-bc-DNA, 7',5'-bc-DNA/DNA and natural DNA duplexes were then investigated by molecular dynamics simulations on a $60 \mathrm{~ns}$ trajectory. The atomic charges of the four nucleoside units were calculated using the R.E.D. III.5 tools package ${ }^{[30]}$ and were added into the Amber94 force field. 7',5'-bc-DNA strands were constructed starting from natural DNA in B-conformation. Simulation of the 7',5'-bc-DNA homo duplex showed a rapid convergence toward a stable right handed helix (Figure 6a). This structure possesses an overall shape relatively close to natural B-DNA (Figure 6c), despite a slightly less coiled helix (average of 12.8 bases per turn for $7^{\prime}, 5^{\prime}$ bc-DNA and 11.4 for DNA) and a significantly wider minor groove (13.2 $\AA$ for 7',5'-bc-DNA and $6.7 \AA$ for DNA). Compared to DNA, the $7^{\prime}, 5^{\prime}$-bc-DNA homo-duplex seems to be stabilized by an increased base-stacking area with a calculated average overlap between bases of $9.40 \AA^{2}$ for $7^{\prime}, 5^{\prime}-$ bc-DNA and $6.77 \AA^{2}$ for DNA.

In the 7',5'-bc-DNA homo-duplex the nucleosides surprisingly adopt alternating C6'-exo and C6'-endo conformations along the nucleotide chain in both strands. As a consequence, the $y$ angles switch between the $+S C$ and ap arrangement in an alternating manner (Figure $6 \mathrm{~g}$ ), differing strongly from the expected regular $+s c$ arrangement found in natural DNA duplex (Figure 6i). This alternating pattern is also apparent in the pseudorotation phase angle $P$ adopted by the furanose rings (Figure 6j), leading to a O4'-endo, C1'-exo pucker for the C6'-endo conformers, or a C1'-exo, C2'-endo pucker for the $\mathrm{C6}^{\prime}$-exo conformers. Interestingly, the conformational restriction induced by the bicyclic structure is well reflected in the distribution of the pseudorotation phase angles. While the more flexible 2'-deoxynucleosides span a wide spectrum of $P$-angles (Figure 6k), the 7',5'-bc-nucleosides are locked into two discrete populations. This is in agreement with the reduced entropy contribution to the free enthalpy of duplex formation as calculated from the UV-melting curves.

In the hybrid 7',5'-bc-DNA/DNA duplex, the more rigid bc-DNA strand adopts a conformation similar as in the fully modified system. Interestingly, the DNA strand also shows a partial switch of the $\gamma$ angles to the non-canonical ap arrangement (Figure 6h) highlighting the adaptability of the more flexible DNA-strand to the more rigid 7',5'-bc-DNA strand. The need for conformational adaption of the DNA backbone may also explain the difficulties in cross-pairing between the two backbone types.

To find a rationale for the differential effect of single substitutions of $7^{\prime}, 5^{\prime}$-bc-nucleosides with different bases on duplex stability, we also performed molecular dynamics simulations on duplexes of DNA with ON2, ON5, ON6 and ON7 (see Supp. Inf., Figure S4). A correlation could be observed between 7',5'-bc-nucleosides favoring the C6'-exo conformation and an increase in duplex stability (Figure 7). These data demonstrate that a displacement of the backbone linkage from the $3^{\prime}$ to the 7 ' position, and in consequence alterations of the $\varepsilon$ and $\zeta$ angles (denominated in analogy to the DNA structural descriptors), is well tolerated when the $\gamma$ angle is conserved in a natural arrangement. But such modifications 
become destabilizing when the $\gamma$ angle is oriented in an ap arrangement. Therefore, the orientation of the $\gamma$ angle could be an important parameter for developing nucleic acid like base-pairing systems that are orthogonal to DNA.

\section{Conclusions}

Inspired by the 2',5'-DNA system we decided to explore an alternative, unnatural DNA topology in which the point of attachment of the nucleotide unit is shifted from the $3^{\prime}$-position to the 7'-position (7',5'-bc-DNA). This topological shift is unique to the scaffold of bicyclo-DNA and cannot be attained by the natural ribofuranose structure. We have succeeded in finding a convenient synthetic access to the nucleoside building blocks of $7^{\prime}, 5^{\prime}$-bc-DNA with all canonical nucleobases as well as to the corresponding oligonucleotides. Single substitutions in DNA/DNA or DNA/RNA duplexes with this modification do generally not inflict strongly with duplex stability, indicating that isolated structural perturbations arising from the shift of the phosphodiester attachment are relatively well tolerated and compensated by the surrounding natural nucleotides. This is somehow different from single substitutions with the simple $3^{\prime}$-methylene homologue of DNA, ${ }^{[23]}$ which shows substantial destabilization, most likely due to the missing conformational restriction as compared to $7^{\prime}, 5^{\prime}$ DNA. The accuracy of base-pairing is also maintained in these cases, as mismatch formation is associated with similar destabilization of duplexes as for natural DNA.

More of interest is the pairing behavior of fully modified $7^{\prime}, 5^{\prime}$-bc-DNA. The affinity of such oligonucleotides to complementary DNA and RNA is strongly reduced to inexistent while 7',5'-homo duplex formation readily occurs with similar thermal and thermodynamic characteristics as for DNA. Similar to the case of natural DNA, only antiparallel strand alignment is tolerated in duplexes. This is not obvious given the quasi C2 symmetry of the 7'-, and 5'-substituents along a pseudo symmetry axis running through the centers C1' and C6' and thus lying in the plane of a base-pair.

A structural rationalization of the differences in stability of homo- and hetero duplexes can be inferred from molecular modeling studies that revealed a pattern of alternative 6'exo/6'-endo conformations in consecutive nucleotides of the bicyclic scaffold. This backbone structure seems to be relatively rigid and also occurs in mixed, 7',5'-bc-DNA/DNA duplexes. In these mixed duplexes, the 7',5'-bc-DNA strand drives the DNA complement in a higher energy conformation in which the torsion angles $\gamma$ of consecutive nucleotides partially oscillate between the $+\mathrm{sc}$ and the ap arrangement, resulting in a drop of duplex stability.

Thus, 7',5'-bc-DNA constitutes an artificial genetic system that can still weakly crosspair with natural nucleic acids but that shows a competitive preference for complements of its own backbone type. This promiscuity may be exploited in the sense that genetic information can be transferred from the real DNA world into an unnatural 7',5'-bc-DNA world provided there exists a set of DNA polymerases that accept both genetic building blocks as substrates and operate in a read and write mode. This could then be the entry into two orthogonal genetic systems that could be manipulated independently.

\section{Experimental Section}


General procedures: All reactions were performed in dried glassware and under an inert atmosphere of Argon. Anhydrous solvents for reactions were obtained by filtration through activated alumina or by storage over molecular sieves (4 $\AA$ ). Colum chromatography (CC) was performed on silica gel (SiliaFlash P60, 40-63 um, $60 \AA$ ). Methanol used for CC was of HPLC grade, all other solvents used for CC were of technical grade and distilled prior to use. Thin-layer chromatography was performed on silica gel plates (macherey-nagel, precoated TLC-plates sil G-25 UV254). Compounds were visualized under UV-light or by dipping in a $p$-Anisaldehyde staining solution [ $p$ Anisaldehyde $(3.7 \mathrm{~mL})$, glacial acetic acid $(3.7 \mathrm{~mL})$, concd sulfuric acid $(5 \mathrm{~mL})$, ethanol (135 mL)] followed by heating with a heat gun. NMR spectra were recorded at 300 or $400 \mathrm{MHz}\left({ }^{1} \mathrm{H}\right)$, at 75 or $101 \mathrm{MHz}\left({ }^{13} \mathrm{C}\right)$ and at $122 \mathrm{MHz}\left({ }^{31} \mathrm{P}\right)$ in either $\mathrm{CDCl}_{3}, \mathrm{CD}_{3} \mathrm{OD},\left(\mathrm{CD}_{3}\right)_{2} \mathrm{SO}$ or $\mathrm{CD}_{3} \mathrm{CN}$. Chemical shifts $(\delta)$ are reported relative to the residual undertreated solvent peak $\left[\mathrm{CDCl}_{3}: 7.26 \mathrm{ppm}\left({ }^{1} \mathrm{H}\right), 77.16 \mathrm{ppm}\left({ }^{13} \mathrm{C}\right)\right.$; $\mathrm{CD}_{3} \mathrm{OD}: 3.31 \mathrm{ppm}\left({ }^{1} \mathrm{H}\right)$; $\left(\mathrm{CD}_{3}\right)_{2} \mathrm{SO}: 2.50 \mathrm{ppm}\left({ }^{1} \mathrm{H}\right), 39.52 \mathrm{ppm}\left({ }^{13} \mathrm{C}\right)$; $\left.\mathrm{CD}_{3} \mathrm{CN}: 1.94 \mathrm{ppm}\left({ }^{1} \mathrm{H}\right)\right]$. Signal assignments are based $\mathrm{APT}$ and DEPT and on ${ }^{1} \mathrm{H},{ }^{1} \mathrm{H}$ and ${ }^{1} \mathrm{H},{ }^{13} \mathrm{C}$ correlation experiments (COSY, HSQC, HMBC). High resolution mass detections were performed by electrospray ionization in the positive mode (ion trap, $\mathrm{ESI}^{+}$).

Ethyl (E and Z, 1'R,5'S, 7'R)-(7'-hydroxy-3',3'-dimethyl-2',4'-dioxabicyclo[3.3.0]oct-6'-ylidene)acetate (2a/b): A solution of the epoxide 1 $(4.46 \mathrm{~g}, 18.4 \mathrm{mmol})$ in dry THF $(100 \mathrm{~mL})$ was cooled down to $-78^{\circ} \mathrm{C}$. Then LiHMDS (1M in THF, $\left.22.1 \mathrm{~mL}, 22.1 \mathrm{mmol}\right)$ was slowly added. The solution was stirred for 2 hours at $-78^{\circ} \mathrm{C}$ before being allowed to warm to it and neutralized with addition of $1 \mathrm{M}$ aqueous $\mathrm{HCl}(22.1$ $\mathrm{mL})$. The mixture was then diluted with EtOAc $(100 \mathrm{~mL})$ and THF was removed under reduced pressure. The mixture was then washed with $0.5 \mathrm{M} \mathrm{NaH}_{2} \mathrm{PO}_{4}(50 \mathrm{~mL})$ and aqueous phase extracted with EtOAc $(2 \times 50 \mathrm{~mL})$. The combined organic phases were dried over $\mathrm{MgSO}_{4}$, filtered and evaporated. The crude product was purified by CC (EtOAc/hexane $3: 1$ ) to yield the two isomers $2 \mathrm{a} / \mathrm{b}$ (3.30 g, $74 \%$ ) as a slightly yellow solid. Compound $2 \mathrm{a}: R_{\mathrm{f}}=0.37$ (EtoAc/hexane 1:1); ${ }^{1} \mathrm{H} \mathrm{NMR}(300 \mathrm{MHz}, \mathrm{CDCl}) \delta 6.07-5.98(\mathrm{~m}, 1 \mathrm{H}, \mathrm{H}-\mathrm{C}(2)), 5.59$ $\left(\mathrm{d}, J=6.0 \mathrm{~Hz}, 1 \mathrm{H}, \mathrm{H}-\mathrm{C}\left(5^{\prime}\right)\right), 4.94-4.81\left(\mathrm{~m}, 1 \mathrm{H}, \mathrm{H}-\mathrm{C}\left(1^{\prime}\right)\right), 4.65\left(\mathrm{t}, J=5.6 \mathrm{~Hz}, 1 \mathrm{H}, \mathrm{H}-\mathrm{C}\left(7^{\prime}\right)\right), 4.18\left(\mathrm{q}, J=7.1 \mathrm{~Hz}, 2 \mathrm{H}, \mathrm{CH}_{3} \mathrm{CH} \mathrm{H}_{2}\right), 2.67(b r$, $1 \mathrm{H}, \mathrm{OH}), 2.37$ (dd, $\left.J=13.5,7.5 \mathrm{~Hz}, 1 \mathrm{H}, \mathrm{H}-\mathrm{C}\left(8^{\prime}\right)\right), 1.55-1.42\left(\mathrm{~m}, 1 \mathrm{H}, \mathrm{H}-\mathrm{C}\left(8^{\prime}\right)\right), 1.40,1.33\left(2 \mathrm{~s}, 6 \mathrm{H},\left(\mathrm{CH}_{3}\right){ }_{2} \mathrm{C}\right), 1.26(\mathrm{t}, J=7.1 \mathrm{~Hz}, 3 \mathrm{H}$, $\left.\mathrm{CH}_{2} \mathrm{CH}_{3}\right) ;{ }^{13} \mathrm{C}$ NMR (75 MHz, $\left.\mathrm{CDCl}_{3}\right) \delta 165.75$ (C(1)), 161.61 (C(6’)), 116.53 (C(2)), 110.69 (C(3')), 76.55 (C(5')), 75.52 (C(1')), 71.63 $\left(\mathrm{C}\left(7^{\prime}\right)\right), 60.51\left(\mathrm{CH}_{2} \mathrm{CH}_{3}\right), 37.46\left(\mathrm{C}\left(8^{\prime}\right)\right), 26.44,24.11\left(\left(\mathrm{CH}_{3}\right)_{2} \mathrm{C}\right), 14.27\left(\mathrm{CH}_{2} \mathrm{CH}_{3}\right) ; \mathrm{ESI}{ }^{+}-\mathrm{HRMS} / z$ calcd for $\mathrm{C}_{12} \mathrm{H}_{19} \mathrm{O}_{5}\left(\left[\mathrm{M}+\mathrm{H}^{+}\right) 243.1227\right.$, found 243.1231; Compound 2b: $R_{\mathfrak{f}}=0.52\left(\right.$ EtoAc/hexane 1:1); ${ }^{1} \mathrm{H}$ NMR $\left(300 \mathrm{MHz}, \mathrm{CDCl}_{3}\right) \delta 6.15-6.05(\mathrm{~m}, 1 \mathrm{H}, \mathrm{H}-\mathrm{C}(2)), 5.37-5.02(\mathrm{~m}$, $\left.2 \mathrm{H}, \mathrm{H}-\mathrm{C}\left(5^{\prime}\right), \mathrm{OH}\right), 4.87\left(\mathrm{~d}, J=3.4 \mathrm{~Hz}, 1 \mathrm{H}, \mathrm{H}-\mathrm{C}\left(1^{\prime}\right)\right), 4.67\left(\mathrm{t}, J=4.9 \mathrm{~Hz}, 1 \mathrm{H}, \mathrm{H}-\mathrm{C}\left(7^{\prime}\right)\right), 4.20\left(\mathrm{qd}, J=7.1,0.9 \mathrm{~Hz}, 2 \mathrm{H}, \mathrm{CH}_{3} \mathrm{CH} \mathrm{H}_{2}\right), 2.55(\mathrm{dd}, J=$ 14.6, 8.1 Hz, $\left.1 \mathrm{H}, \mathrm{H}-\mathrm{C}\left(8^{\prime}\right)\right), 1.94-1.77\left(\mathrm{~m}, 1 \mathrm{H}, \mathrm{H}-\mathrm{C}\left(8^{\prime}\right)\right), 1.39-1.25\left(\mathrm{~m}, 9 \mathrm{H},\left(\mathrm{CH}_{3}\right)_{2} \mathrm{C}, \mathrm{CH}_{2} \mathrm{CH}_{3}\right) ;{ }^{13} \mathrm{C} \mathrm{NMR}\left(75 \mathrm{MHz}, \mathrm{CDCl}_{3}\right) \delta 167.91$ $(\mathrm{C}(1)), 167.43\left(\mathrm{C}\left(6^{\prime}\right)\right), 120.13(\mathrm{C}(2)), 111.75\left(\mathrm{C}\left(3^{\prime}\right)\right), 81.62\left(\mathrm{C}\left(5^{\prime}\right)\right), 78.08\left(\mathrm{C}\left(1^{\prime}\right)\right), 70.85\left(\mathrm{C}\left(7^{\prime}\right)\right), 61.25\left(\mathrm{CH}_{2} \mathrm{CH}_{3}\right), 36.53\left(\mathrm{C}\left(8^{\prime}\right)\right), 27.38$, $25.45\left(\left(\mathrm{CH}_{3}\right)_{2} \mathrm{C}\right), 14.19\left(\mathrm{CH}_{2} \mathrm{CH}_{3}\right)$; ESI+-HRMS $\mathrm{m} / \mathrm{z}$ calcd for $\mathrm{C}_{12} \mathrm{H}_{19} \mathrm{O}_{5}\left([\mathrm{M}+\mathrm{H}]^{+}\right)$243.1227, found 243.1227.

Ethyl (1'R, 5'S, 6'S, 7'R)-(7'-hydroxy-3',3'-dimethyl-2',4'-dioxabicyclo[3.3.0]oct-6'-yl)acetate (3): To a solution of the alcohols 2a/b (12.65 g, $52.2 \mathrm{mmol}$ ) and nickel chloride hexahydrate ( $2.48 \mathrm{~g}, 10.4 \mathrm{mmol})$ in $\mathrm{EtOH}(300 \mathrm{~mL})$ was added portionwise sodium borohydride ( $9.88 \mathrm{~g}$, $261 \mathrm{mmol}$ ) at $0^{\circ} \mathrm{C}$. The resulting dark solution was stirred for $30 \mathrm{~min}$ at $0^{\circ} \mathrm{C}$ and 90 min at rt. Then $\mathrm{EtOH}$ was carefully concentrated under reduced pressure, the resulting solid diluted with EtOAc $(200 \mathrm{~mL})$ and the excess of $\mathrm{NaBH}_{4}$ quenched by addition of water (100 $\mathrm{mL}$ ) at $0^{\circ} \mathrm{C}$ followed by stirring at $\mathrm{rt}$ for $30 \mathrm{~min}$. The two phases were then separated. Organic phase was washed with water (100 $\mathrm{mL}$ ). Aqueous phases were then combined, filtered and extracted with EtOAc $(2 \times 100 \mathrm{~mL})$. The combined organic phases were dried over $\mathrm{MgSO}_{4}$, filtered and concentrated. The crude product was purified by CC (EtOAc/hexane 2:1) to yield 3 (11.4 g, $90 \%$ ) as a white solid. Data for 3: $R_{\mathrm{f}}=0.40\left(\right.$ EtOAc/hexane 1:1); ${ }^{1} \mathrm{H}$ NMR $\left(300 \mathrm{MHz}, \mathrm{CDCl}_{3}\right) \delta 4.65-4.52\left(\mathrm{~m}, 2 \mathrm{H}, \mathrm{H}-\mathrm{C}\left(1^{\prime}\right), \mathrm{H}-\mathrm{C}\left(5^{\prime}\right)\right), 4.15(\mathrm{qd}, \mathrm{J}=7.1,1.4 \mathrm{~Hz}$, $2 \mathrm{H}, \mathrm{CH}_{3} \mathrm{CH}_{2}$ ), 4.05 (ddd, $\left.J=10.0,9.99,6.2 \mathrm{~Hz}, 1 \mathrm{H}, \mathrm{H}-\mathrm{C}\left(7^{\prime}\right)\right), 2.86(b r, \mathrm{~s}, 1 \mathrm{H}, \mathrm{OH}), 2.65(\mathrm{qd}, J=16.9,7.1 \mathrm{~Hz}, 2 \mathrm{H}, \mathrm{H}-\mathrm{C}(2)), 2.24(\mathrm{dd}, J=$ 13.7, 6.2 Hz, 1H, H-C(8')), 1.93 (dt, J=12.7, 7.1 Hz, 1H. H-C(6')), $1.56\left(\mathrm{ddd}, J=13.9,10.2,5.5 \mathrm{~Hz}, 1 \mathrm{H}, \mathrm{H}-\mathrm{C}\left(8^{\prime}\right)\right), 1.38\left(\mathrm{~s}, 3 \mathrm{H},(\mathrm{CH})_{2} \mathrm{C}\right)$, $1.30-1.21\left(\mathrm{~m}, 6 \mathrm{H},\left(\mathrm{CH}_{3}\right)_{2} \mathrm{C}, \mathrm{CH}_{2} \mathrm{CH}_{3}\right) ;{ }^{13} \mathrm{C} \mathrm{NMR}\left(75 \mathrm{MHz}, \mathrm{CDCl}_{3}\right) \delta 174.38(\mathrm{C}(1)), 109.06\left(\mathrm{C}\left(3^{\prime}\right)\right), 79.65\left(\mathrm{C}\left(5^{\prime}\right)\right), 77.19\left(\mathrm{C}\left(1^{\prime}\right), 74.32\right.$ (C(7’), $60.80\left(\mathrm{CH}_{2} \mathrm{CH}_{3}\right), 46.66\left(\mathrm{C}\left(6^{\prime}\right)\right), 40.38\left(\mathrm{C}\left(8^{\prime}\right)\right), 32.43(\mathrm{C}(2)), 26.00,23.69\left(\left(\mathrm{CH}_{3}\right)_{2} \mathrm{C}\right), 14.17\left(\mathrm{CH}_{2} \mathrm{CH}_{3}\right)$; ESI+-HRMS m/z calcd for $\mathrm{C}_{12} \mathrm{H}_{21} \mathrm{O}_{5}\left([\mathrm{M}+\mathrm{H}]^{+}\right)$245.1384, found 245.1388. 
Ethyl (1'R, 5'S, 6'S, 7'R)-(7'-(tert-butyldiphenylsilyl)oxy)-3', 3'-dimethyl-2;'4'-dioxabicyclo[3.3.0]oct-6'-yl)acetate (4): To a solution of the alcohol $3(2.50 \mathrm{~g}, 10.2 \mathrm{mmol})$, N-methylimidazole $(12.6 \mathrm{~g}, 153 \mathrm{mmol})$ and iodine $(7.80 \mathrm{~g}, 30.6 \mathrm{mmol})$ in dry THF $(60 \mathrm{~mL})$ was added dropwise tert-butyl(chloro)diphenylsilane $(3.0 \mathrm{~mL}, 11.2 \mathrm{mmol})$ at $\mathrm{rt}$. The solution was stirred for 3 hours at rt and then THF was evaporated, the mixture diluted with EtOAc $(50 \mathrm{~mL})$ and washed with $10 \%$ aqueous $\mathrm{Na}_{2} \mathrm{O}_{3} \mathrm{~S}_{2}(2 \mathrm{X} 40 \mathrm{~mL})$. Aqueous phases were then combined and extracted with EtOAc $(50 \mathrm{~mL})$. The combined organic phases were dried over $\mathrm{MgSO}_{4}$, filtered and evaporated. The crude product was purified by CC (EtOAc/hexane 1:10) to yield $4\left(5.01 \mathrm{~g}\right.$, quantitative yield) as a white solid. Data for 4: $R_{\mathrm{f}}=0.87(\mathrm{DCM} / \mathrm{MeOH}$ 10:1); ${ }^{1} \mathrm{H}$ NMR (300 MHz, CDCl 3 ) $\delta 7.77-7.59$ (m, 4H, H-arom), $7.51-7.32$ (m, 6H, H-arom), 4.61 (t, J=5.7 Hz, 1H, H-C(5')), $4.49(\mathrm{t}, J$ $\left.=5.7 \mathrm{~Hz}, 1 \mathrm{H}, \mathrm{H}-\mathrm{C}\left(1^{\prime}\right)\right), 4.15$ (q, $J=6.9 \mathrm{~Hz}, 2 \mathrm{H}, \mathrm{CH}_{3} \mathrm{CH}_{2}$ ), 3.96 (dd, $\left.J=15.5,9.5 \mathrm{~Hz}, 1 \mathrm{H}, \mathrm{H}-\mathrm{C}\left(7^{\prime}\right)\right), 2.64-2.32(\mathrm{~m}, 2 \mathrm{H}, \mathrm{H}-\mathrm{C}(2)), 2.15$ (tt, $J$ = 9.0, 4.3 Hz, 1H, H-C(6')), $1.83\left(\mathrm{dd}, J=12.7,5.2 \mathrm{~Hz}, 1 \mathrm{H}, \mathrm{H}-\mathrm{C}\left(8^{\prime}\right)\right), 1.61-1.45\left(\mathrm{~m}, 1 \mathrm{H}, \mathrm{H}-\mathrm{C}\left(8^{\prime}\right)\right), 1.27$ (td, J = 7.1, $\left.1.9 \mathrm{~Hz}, 3 \mathrm{H}, \mathrm{CH}_{2} \mathrm{CH}_{3}\right)$, $1.18\left(\mathrm{~s}, 6 \mathrm{H},\left(\mathrm{CH}_{3}\right)_{2} \mathrm{C}\right), 1.09,1.08\left(2 \mathrm{~s}, 9 \mathrm{H},\left(\mathrm{CH}_{3}\right)_{3}-\mathrm{C}-\mathrm{Si}\right) ;{ }^{13} \mathrm{C} \mathrm{NMR}\left(75 \mathrm{MHz}, \mathrm{CDCl}_{3}\right) \delta 173.07(\mathrm{C}(1)), 135.87,135.85(\mathrm{CH}-\operatorname{arom}), 134.08$,

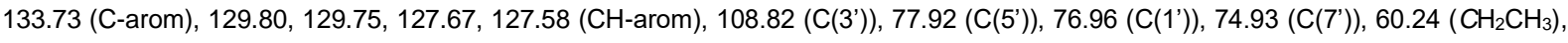
$47.27\left(\mathrm{C}\left(6^{\prime}\right)\right), 40.27\left(\mathrm{C}\left(8^{\prime}\right)\right)$, $\left.\left.31.10(\mathrm{C}(2)), 27.04\left(\mathrm{CH}_{3}\right)_{3}-\mathrm{C}-\mathrm{Si}\right), 25.86\left(\left(\mathrm{CH}_{3}\right)_{2} \mathrm{C}\right), 23.83\left(\left(\mathrm{CH}_{3}\right)_{2} \mathrm{C}\right), 19.23\left(\mathrm{CH}_{3}\right)_{3}-\mathrm{C}-\mathrm{Si}\right), 14.24\left(\mathrm{CH}_{2}-\mathrm{CH}_{3}\right)$; ESI+-HRMS $m / z$ calcd for $\mathrm{C}_{28} \mathrm{H}_{39} \mathrm{O} \mathrm{O}_{5} \mathrm{Si}\left(\left[\mathrm{M}+\mathrm{H}^{+}\right)\right.$483.2561, found 483.2562 .

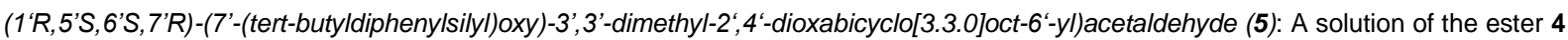
$(8.56 \mathrm{~g}, 16.3 \mathrm{mmol})$ in dry DCM $(120 \mathrm{~mL})$ was cooled down to $-78^{\circ} \mathrm{C}$ and then DiBAL-H (1M in cyclohexane, $\left.18 \mathrm{~mL}, 18 \mathrm{mmol}\right)$ was slowly added. The solution was further stirred at $-78^{\circ} \mathrm{C}$ for 90 min before being allowed to warm to rt. Reaction was quenched by addition of 0.5 $\mathrm{M}$ aqueous $\mathrm{NaH}_{2} \mathrm{PO}_{4}(100 \mathrm{~mL})$. The organic phase was separated and aqueous phase was further extracted with $\mathrm{DCM}(2 \mathrm{X} 100 \mathrm{~mL})$. The combined organic phases were dried over $\mathrm{MgSO}_{4}$, filtered and evaporated. The crude product was purified by CC (EtOAc/hexane 2:10 to 2:1) to yield 5 (6.36 g, 89\%). Data for $\mathbf{5}: R_{\mathrm{f}}=0.65$ (EtOAc/hexane 2:1); ${ }^{1} \mathrm{H}$ NMR $\left(300 \mathrm{MHz}, \mathrm{CDCl}_{3}\right) \delta 9.72(\mathrm{~s}, 1 \mathrm{H}, \mathrm{H}-\mathrm{C}(1)), 7.65$ (td, $J=8.0,1.6 \mathrm{~Hz}, 4 \mathrm{H}, \mathrm{H}$-arom), $7.47-7.33\left(\mathrm{~m}, 6 \mathrm{H}, \mathrm{H}\right.$-arom), 4.57 (t, $\left.J=5.7 \mathrm{~Hz}, 1 \mathrm{H}, \mathrm{H}-\mathrm{C}\left(5^{\prime}\right)\right), 4.51\left(\mathrm{t}, J=5.7 \mathrm{~Hz}, 1 \mathrm{H}, \mathrm{H}-\mathrm{C}\left(1^{\prime}\right)\right), 3.99(\mathrm{td}$, $\left.J=10.0,5.9 \mathrm{~Hz}, 1 \mathrm{H}, \mathrm{H}-\mathrm{C}\left(7^{\prime}\right)\right), 2.58-2.43(\mathrm{~m}, 2 \mathrm{H}, \mathrm{H}-\mathrm{C}(2)), 2.20-2.08\left(\mathrm{~m}, 1 \mathrm{H}, \mathrm{H}-\mathrm{C}\left(6^{\prime}\right)\right), 1.87\left(\mathrm{dd}, J=13.5,5.9 \mathrm{~Hz}, 1 \mathrm{H}, \mathrm{H}-\mathrm{C}\left(8^{\prime}\right)\right), 1.53$ (ddd, $\left.J=13.5,10.1,5.5 \mathrm{~Hz}, 1 \mathrm{H}, \mathrm{H}-\mathrm{C}\left(8^{\prime}\right)\right), 1.16\left(\mathrm{~d}, J=3.5 \mathrm{~Hz}, 6 \mathrm{H},\left(\left(\mathrm{CH}_{3}\right)_{2} \mathrm{C}\right), 1.05\right.$ (s, 9H, $\left.\left(\mathrm{CH}_{3}\right)_{3}-\mathrm{C}-\mathrm{Si}\right) ;{ }^{13} \mathrm{C} \mathrm{NMR}\left(75 \mathrm{MHz}, \mathrm{CDCl}_{3}\right) \delta$ 201.87 (C(1)), 135.93, 135.90 (CH-arom), 133.96, 133.73 (C-arom), 129.96, 129.89, 127.79, 127.68 (CH-arom), 108.89 (C(3')), 77.76 $\left(\mathrm{C}\left(5^{\prime}\right)\right), 77.17\left(\mathrm{C}\left(1^{\prime}\right)\right), 74.96\left(\mathrm{C}\left(7^{\prime}\right), 45.44\left(\mathrm{C}\left(6^{\prime}\right)\right), 41.31(\mathrm{C}(2)), 40.16\left(\mathrm{C}\left(8^{\prime}\right)\right), 27.08\left(\mathrm{CH}_{3}\right)_{3}-\mathrm{C}-\mathrm{Si}\right), 25.87\left(\left(\mathrm{CH}_{3}\right)_{2} \mathrm{C}\right), 23.79\left(\left(\mathrm{CH}_{3}\right)_{2} \mathrm{C}\right), 19.25$ $\left.\left(\mathrm{CH}_{3}\right)_{3}-\mathrm{C}-\mathrm{Si}\right)$; $\mathrm{ESI}{ }^{+}-\mathrm{HRMS} \mathrm{m} / \mathrm{z}$ calcd for $\mathrm{C}_{26} \mathrm{H}_{35} \mathrm{O}_{4} \mathrm{Si}\left([\mathrm{M}+\mathrm{H}]^{+}\right)$439.2299, found 439.2297 .

(3aR,4R,6R,6aS)-4-((tert-butyldiphenylsilyl)oxy)-2-methoxyhexahydro-2H-cyclopenta[b]furan-6-ol (6): To a solution of the aldehyde 5 (13.73 g, $31.31 \mathrm{mmol})$ in $\mathrm{MeCN}(170 \mathrm{~mL})$ and $\mathrm{H}_{2} \mathrm{O}(19 \mathrm{~mL})$ was added Indium"l trifluoromethanesulfonate $(703 \mathrm{mg}, 1.25 \mathrm{mmol})$. The solution was stirred for 48 hours, and then solvents were removed under reduced pressure and coevaporated with toluene. The residue was dissolved in dry $\mathrm{MeOH}$ and stirred for 6 hours. After evaporation of solvent, the crude product was purified by $\mathrm{CC}$ (EtOAc/hexane 3:10) to yield a mixture of $6(10.50 \mathrm{~g}, 81 \%)$ in an anomeric ratio $\alpha / \beta \approx 4: 1$ as a colorless oil. Data for $6: R_{\mathrm{f}}=0.53($ EtOAc/hexane $1: 1) ;{ }^{1} \mathrm{H}$ NMR (300 MHz, $\left.\mathrm{CDCl}_{3}\right) \delta 7.63$ (dd, $J=7.1,0.6 \mathrm{~Hz}, 4 \mathrm{H}, \mathrm{H}$-arom), $7.46-7.34$ (m, 6H, H-arom), 4.98 (d, $\left.J=4.8 \mathrm{~Hz}, 0.8 \mathrm{H}, \mathrm{H}-\mathrm{C}(2)\right), 4.91$ (dd, J = 5.9, 1.3 Hz, $0.2 \mathrm{H}, \mathrm{H}-\mathrm{C}(2)), 4.63-4.54(\mathrm{~m}, 1 \mathrm{H}, \mathrm{H}-\mathrm{C}(6 \mathrm{a}))$, $4.53-4.37(\mathrm{~m}, 1 \mathrm{H}, \mathrm{H}-\mathrm{C}(6)), 4.09(\mathrm{~m}, 0.2 \mathrm{H}, \mathrm{H}-\mathrm{C}(4)), 3.92(b r, 0.8 \mathrm{H}$, $\mathrm{H}-\mathrm{C}(4)), 3.29,3.27$ (2s, $3 \mathrm{H}, \mathrm{MeO}), 2.79$ (dd, $J=17.0,8.2 \mathrm{~Hz}, 0.8 \mathrm{H}, \mathrm{H}-\mathrm{C}(3 \mathrm{a})), 2.64-2.51(\mathrm{~m}, 0.2 \mathrm{H}, \mathrm{H}-\mathrm{C}(3 \mathrm{a})), 2.29(\mathrm{~d}, J=8.1 \mathrm{~Hz}, 1 \mathrm{H}$, $\mathrm{OH}$, 2.10 - 1.80 (m, $2.4 \mathrm{H}, \mathrm{H}-\mathrm{C}(3), \mathrm{H}-\mathrm{C}(5)), 1.65$ (ddd, J = 13.2, 9.1, 4.4 Hz, 0.8 H, H-C(5)), $1.44-1.34$ (m, $0.2 \mathrm{H}, \mathrm{H}-\mathrm{C}(3)), 1.22$ (ddd, J $=13.2,8.1,4.9 \mathrm{~Hz}, 0.8 \mathrm{H}, \mathrm{H}-\mathrm{C}(3)), 1.05\left(\mathrm{~s}, 9 \mathrm{H},\left(\mathrm{CH}_{3}\right)_{3}-\mathrm{C}-\mathrm{Si}\right) ;{ }^{13} \mathrm{C} \mathrm{NMR}\left(75 \mathrm{MHz}, \mathrm{CDCl}_{3}\right)$ ठ 135.78, 135.74 (CH-arom), 133.96, 133.84 (Carom), 129.78, 127.72 (CH-arom), 107.21, 106.50 (C(2)), 85.37, 81.76 (C(6a)), 78.11, 77.19 (C(4)), 73.03, 72.44 (C(6)), 55.30, 54.46 (MeO), 50.91, 49.67 (C(3a)), 41.13, 40.29 (C(3)), 38.16, 37.98 (C(5)), 26.96, $\left.\left.26.92\left(\mathrm{CH}_{3}\right)_{3}-\mathrm{C}-\mathrm{Si}\right), 19.07\left(\mathrm{CH}_{3}\right)_{3}-\mathrm{C}-\mathrm{Si}\right)$; ESI+-HRMS m/z calcd for $\mathrm{C}_{26} \mathrm{H}_{35} \mathrm{O}{ }_{4} \mathrm{Si}\left([\mathrm{M}+\mathrm{H}]^{+}\right)$435.1962, found 435.1950 . 
(3aR,4R,6R,6aS)-4-((tert-butyldiphenylsilyl)oxy)-3a,5,6,6a-tetrahydro-4H-cyclopenta[b]furan-6-ol (8): To a solution of the sugar 6 (2.08 g, $5.04 \mathrm{mmol})$ in dry DCM $(35 \mathrm{~mL})$ was added 2,6-lutidine $(2.95 \mathrm{~mL}, 25.2 \mathrm{mmol})$ at $0^{\circ} \mathrm{C}$. After stirring for $20 \mathrm{~min}$ at $0^{\circ} \mathrm{C}$, TMSOTf $(2.73 \mathrm{~mL}$, $15.1 \mathrm{mmol}$ ) was added dropwise, then the solution was allowed to warm to rt and was further stirred for $60 \mathrm{~min}$. The reaction was then quenched by addition of satd $\mathrm{NaHCO}_{3}(40 \mathrm{~mL})$. The organic phase was separated and aqueous phase was further extracted with DCM $\left(3 \times 30 \mathrm{~mL}\right.$ ). The combined organic phases were dried over $\mathrm{MgSO}_{4}$, filtered and evaporated. The resulting product was dissolved in dry THF ( $35 \mathrm{~mL})$, cooled down to $0^{\circ} \mathrm{C}$, and TBAF ( $\left.1 \mathrm{M} \mathrm{in} \mathrm{THF,} 5.6 \mathrm{~mL}, 5.6 \mathrm{mmol}\right)$ was added. After stirring for $10 \mathrm{~min}$ at $0^{\circ} \mathrm{C}$, the solution was diluted with satd $\mathrm{NaHCO}_{3}(30 \mathrm{~mL})$ and extracted with DCM $(4 \times 40 \mathrm{~mL})$. The combined organic phases were dried over $\mathrm{MgSO}_{4}$, filtered and evaporated. The crude product was purified by CC (EtOAc/hexane 1:4) to yield the glycal $8(1.76 \mathrm{~g}, 92 \%)$. Data for $8: R_{\mathrm{f}}=0.49$ (EtOAc/hexane 1:2); ${ }^{1} \mathrm{H}$ NMR (300 MHz, CDCl 3 ) $\delta 7.66$ (m, 4H, H-arom), 7.42 (m, 6H, H-arom), 6.22 (t, $\left.J=2.1 \mathrm{~Hz}, 1 \mathrm{H}, \mathrm{H}-\mathrm{C}(2)\right), 4.91$ (dd, $J=8.2,5.3 \mathrm{~Hz}, 1 \mathrm{H}, \mathrm{H}-\mathrm{C}(3)), 4.70$ (dt, $J=11.1,5.6 \mathrm{~Hz}, 1 \mathrm{H}, \mathrm{H}-\mathrm{C}(6)), 4.56$ (t, $J=2.8 \mathrm{~Hz}, 1 \mathrm{H}, \mathrm{H}-\mathrm{C}(6 \mathrm{a})$ ), 3.97 (d, $J=4.0 \mathrm{~Hz}, 1 \mathrm{H}, \mathrm{H}-$ $\mathrm{C}(4)), 3.24$ (d, J = 8.2 Hz, 1H, H-C(3a)), $2.30(b r, 1 \mathrm{H}, \mathrm{OH}$ ), 2.03 (dd, $J=12.6,5.4 \mathrm{~Hz}, 1 \mathrm{H}, \mathrm{H}-\mathrm{C}(5)), 1.51$ (ddd, $J=12.7,11.2,4.2 \mathrm{~Hz}, 1 \mathrm{H}$, $\mathrm{H}-\mathrm{C}(5)), 1.08$ (s, 9H, $\left.\left(\mathrm{CH}_{3}\right)_{3}-\mathrm{C}-\mathrm{Si}\right) ;{ }^{13} \mathrm{C}$ NMR $\left(75 \mathrm{MHz}, \mathrm{CDCl}_{3}\right) \delta 146.24$ (C(2)), 135.72, 135.69 (CH-arom), 134.03, 133.74 (C-arom), 129.80, 129.78, 127.73 (CH-arom), $101.84(\mathrm{C}(3)), 84.59$ (C(6a)), 76.79(C(4)), $74.10(\mathrm{C}(6)), 55.56(\mathrm{C}(3 \mathrm{a})), 39.38(\mathrm{C}(5)), 26.93\left(\mathrm{CH}_{3}\right)_{3}-\mathrm{C}-$ $\left.\mathrm{Si}), 19.08\left(\mathrm{CH}_{3}\right)_{3}-\mathrm{C}-\mathrm{Si}\right)$; ESI+- $\mathrm{HRMS} \mathrm{m} / z$ calcd for $\mathrm{C}_{23} \mathrm{H}_{29} \mathrm{O}_{3} \mathrm{Si}\left([\mathrm{M}+\mathrm{H}]^{+}\right) 381.1880$, found 381.1893.

(((3aR,4R,6R,6aS)-6-(bis(4-methoxyphenyl)(phenyl)methoxy)-3a,5,6,6a-tetrahydro-4H-cyclopenta[b]furan-4-yl)oxy)(tertbutyl)diphenylsilane (9): To a solution of glycal 8 (1.34 g, $3.52 \mathrm{mmol})$ and DMTr-Cl (1.43 g, $4.23 \mathrm{mmol})$ in a mixture of dry DCM (15 mL) and dry 2,6-lutidine $(15 \mathrm{~mL})$ was added portionwise silver triflate $(1.13 \mathrm{~g}, 4.40 \mathrm{mmol})$, resulting in a deep red suspension. After stirring for 2 hours at rt, an additional portion of $\mathrm{DMTr}-\mathrm{Cl}(239 \mathrm{mg}, 0.705 \mathrm{mmol})$ was added. The suspension was further stirred for 2 hours and then was filtered. The organic phase was washed with satd $\mathrm{NaHCO}_{3}(100 \mathrm{~mL})$ and then the aqueous phase was extracted with $\mathrm{DCM}(3$ $\mathrm{X} 30 \mathrm{~mL}$ ). The combined organic phases were dried over $\mathrm{MgSO}_{4}$, filtered and evaporated. The crude product was purified by $\mathrm{CC}$ $\left(\right.$ EtOAc/hexane 1:7, $\left.+0.5 \% \mathrm{Et}_{3} \mathrm{~N}\right)$ to yield the protected glycal $9(2.24,93 \%)$ as a white foam. Data for 9: $R \mathrm{f}=0.59(\mathrm{EtOAc} / \mathrm{hexane} 1: 2)$; ${ }^{1} \mathrm{H}$ NMR (400 MHz, CDCl 3 ) $\delta 7.76(\mathrm{~d}, J=7.4 \mathrm{~Hz}, 2 \mathrm{H}, \mathrm{H}$-arom), $7.69-7.60$ (m, J = 9.3, 5.9, 4.6 Hz, 8H, H-arom), 7.56 - 7.39 (m, 8H, Harom), 7.33 (t, J=7.3 Hz, 1H, H-arom), $7.00-6.93(\mathrm{~m}, 4 \mathrm{H}, \mathrm{H}$-arom), $6.47-6.37(\mathrm{~m}, 1 \mathrm{H}, \mathrm{H}-\mathrm{C}(2)), 4.67-4.58(\mathrm{~m}, 1 \mathrm{H}, \mathrm{H}-\mathrm{C}(6)), 4.58-$ 4.50 (m, 2H, H-C(3), H-C(6a)), 3.86, $3.85(2 \mathrm{~s}, 6 \mathrm{H}, \mathrm{MeO}), 3.82(\mathrm{~d}, J=4.0 \mathrm{~Hz}, 1 \mathrm{H}, \mathrm{H}-\mathrm{C}(4)), 3.08(\mathrm{~d}, J=8.1 \mathrm{~Hz}, 1 \mathrm{H}, \mathrm{H}-\mathrm{C}(3 \mathrm{a})), 1.67$ (td, J = 12.4, 4.2 Hz, 1H, H-C(5)), 1.28 (dd, J=12.7, $5.4 \mathrm{~Hz}, 1 \mathrm{H}, \mathrm{H}-\mathrm{C}(5)), 1.11$ (s, 9H, $\left.\left.\left(\mathrm{CH}_{3}\right)_{3}-\mathrm{C}-\mathrm{Si}\right) ;{ }^{3} \mathrm{C} \mathrm{NMR}(75 \mathrm{MHz}, \mathrm{CDCl})\right) \delta 158.67(\mathrm{MeO}-$ C-arom), 147.61 (C(2)), 146.26, 137.36, 137.21 (C-arom), 135.81, 135.78 (CH-arom), 134.17, 134.04 (C-arom), 130.48, 129.83, 129.81, 128.37, 127.98, 127.76, 127.73, 126.79, 113.32, 113.28 (CH-arom), $100.29(\mathrm{C}(3)), 86.96(C(\mathrm{Ph}) 3), 84.95(\mathrm{C}(6 \mathrm{a})), 76.17(\mathrm{C}(6))$,

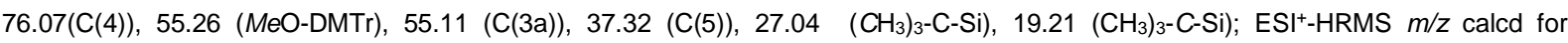
$\mathrm{C}_{44} \mathrm{H}_{46} \mathrm{O}_{5} \mathrm{NaSi}\left([\mathrm{M}+\mathrm{Na}]^{+}\right)$705.3007, found 705.3021.

(3aS,4R,6R,6aS)-6-(bis(4-methoxyphenyl)(phenyl)methoxy)-3a,5,6,6a-tetrahydro-4H-cyclopenta[b]furan-4-ol (10): To a solution of glycal $9(2.23 \mathrm{~g}, 3.27 \mathrm{mmol})$ in dry THF $(20 \mathrm{~mL})$ was added TBAF (1M in THF, $20 \mathrm{~mL}, 20 \mathrm{mmol})$ at rt. The solution was stirred for $20 \mathrm{~h}$ and then was diluted with satd $\mathrm{NaHCO}_{3}(100 \mathrm{~mL})$ and extracted with $\mathrm{DCM}(3 \times 80 \mathrm{~mL})$. The combined organic phases were dried over MgSO 4 , filtered and evaporated. The crude product was purified by $\mathrm{CC}\left(0.5 \% \mathrm{MeOH}\right.$ in $\left.\mathrm{DCM},+0.5 \% \mathrm{Et}_{3} \mathrm{~N}\right)$ to yield 10 (1.45 g, quant.) as a white foam. Data for 10: $R_{\mathrm{f}}=0.44(\mathrm{EtOAc} / \mathrm{hexane} 1: 1) ;{ }^{1} \mathrm{H} \mathrm{NMR}\left(300 \mathrm{MHz}, \mathrm{CDCl}_{3}\right) \delta 7.53-7.46(\mathrm{~m}, 2 \mathrm{H}, \mathrm{H}-\mathrm{arom}), 7.43-7.35(\mathrm{~m}, 4 \mathrm{H}, \mathrm{H}-$ arom), 7.21 (dd, J = 10.7, $5.3 \mathrm{~Hz}, 2 \mathrm{H}, \mathrm{H}$-arom), $7.16-7.08$ (m, 1H, H-arom), $6.80-6.71$ (m, 4H, H-arom), 6.30 (t, J=2.1 Hz, $1 \mathrm{H}, \mathrm{H}$ $\mathrm{C}(2)), 4.68$ (t, J=2.8 Hz, 1H, H-C(3)), $4.29-4.14(\mathrm{~m}, 2 \mathrm{H}, \mathrm{H}-\mathrm{C}(6), \mathrm{H}-\mathrm{C}(6 \mathrm{a})), 3.71$ (s, 6H, MeO), 3.65 (d, J = 3.5 Hz, $1 \mathrm{H}, \mathrm{H}-\mathrm{C}(4)), 2.87$ (d, $J=7.9 \mathrm{~Hz}, 1 \mathrm{H}, \mathrm{H}-\mathrm{C}(3 \mathrm{a})), 1.59$ (ddd, $J=13.2,11.6,4.3 \mathrm{~Hz}, 1 \mathrm{H}, \mathrm{H}-\mathrm{C}(5)), 1.05-0.95(\mathrm{~m}, 2 \mathrm{H}, \mathrm{H}-\mathrm{C}(5), \mathrm{OH}) ;{ }^{13} \mathrm{C} \mathrm{NMR}(75 \mathrm{MHz}, \mathrm{CDCl} 3) \delta$ 158.54 (MeO-C-arom), 147.64 (C(2)), 145.82, 137.12, 137.08 (C-arom), 130.26, 128.29, 127.81, 126.71, 113.13 (CH-arom), 100.17 (C(3)), $\left.86.75\left(C(\mathrm{Ph})_{3}\right), 84.42 \mathrm{C}(6 \mathrm{a})\right)$, $75.54(\mathrm{C}(6))$, $74.59(\mathrm{C}(4)), 55.22$ (MeO-DMTr), 54.25 (C(3a)), $37.56(\mathrm{C}(5))$; ESI+-HRMS m/z calcd for $\mathrm{C}_{30} \mathrm{H}_{27} \mathrm{O}_{5}\left([\mathrm{M}+\mathrm{H}]^{+}\right)$467.1853, found 467.1844. 
$\left(3^{\prime} S, 5^{\prime} R, 7^{\prime} R\right)-1-\left\{2^{\prime}, 3^{\prime}-D i d e o x y-3,5^{\prime}-e t h a n o-7^{\prime}-h y d r o x y-5^{\prime}-O-\left[\left(4,4^{\prime}\right.\right.\right.$-dimethoxytriphenyl)methyl]- $\beta$-D-ribofuranosyl\} thymine (11): To a solution of glycal $10(1.45 \mathrm{~g}, 3.27 \mathrm{mmol})$ in dry DCM $(45 \mathrm{~mL})$, at $0^{\circ}$, was added dropwise BSA $(2.0 \mathrm{~mL}, 8.18 \mathrm{mmol})$ and then the solution was allowed to warm to rt. After stirring for $45 \mathrm{~min}$, Thymine $(595 \mathrm{mg}, 4.91 \mathrm{mmol})$ was added and the reaction was further stirred for 60 $\mathrm{min}$ at rt. The mixture was then cooled down to $0^{\circ} \mathrm{C}$ and $\mathrm{N}$-iodosuccinimide $(875 \mathrm{mg}, 3.92 \mathrm{mmol})$ was added. After stirring for $3 \mathrm{~h}$ at $0^{\circ} \mathrm{C}$ and for $4 \mathrm{~h}$ at rt, the reaction mixture was diluted with EtOAc $(100 \mathrm{~mL})$ and subsequently washed with a $10 \%$ aq solution of $\mathrm{Na}_{2} \mathrm{~S}_{2} \mathrm{O}_{3}$ $(100 \mathrm{~mL})$ and satd $\mathrm{NaHCO}_{3}(100 \mathrm{~mL})$. Aqueous phases were combined and extracted with $\mathrm{DCM}(3 \mathrm{X} 50 \mathrm{~mL})$. The combined organic phases were dried over $\mathrm{MgSO}_{4}$, filtered and evaporated. The crude product was dissolved in dry toluene $(45 \mathrm{~mL})$ and then $\mathrm{Bu}_{3} \mathrm{SnH}$ (1.32 $\mathrm{mL}, 4.91 \mathrm{mmol}$ ) and azoisobutyronitrile (AIBN, $53 \mathrm{mg}, 0.33 \mathrm{mmol}$ ) were added at rt. After heating at $70^{\circ} \mathrm{C}$ for $30 \mathrm{~min}$, the mixture was cooled down to rt and TBAF was added (1M in THF, $6.5 \mathrm{~mL}, 6.5 \mathrm{mmol})$. The solution was further stirred for $25 \mathrm{~min}$ and was diluted with satd $\mathrm{NaHCO}_{3}(100 \mathrm{~mL})$ and extracted with $\mathrm{DCM}(4 \times 70 \mathrm{~mL})$. The combined organic phases were dried over $\mathrm{MgSO}_{4}$, filtered and evaporated. The crude product was purified by CC $\left(3 \% \mathrm{MeOH}\right.$ in DCM, $\left.+0.5 \% \mathrm{Et}_{3} \mathrm{~N}\right)$ to yield $11(1.45 \mathrm{~g}, 73 \%$ over two steps) as a white foam. Data for 11: $R_{\mathrm{f}}=0.29\left(6 \% \mathrm{MeOH}\right.$ in DCM); ${ }^{1} \mathrm{H} \mathrm{NMR}\left(400 \mathrm{MHz}, \mathrm{CDCl}_{3}\right) \delta 9.37(\mathrm{br}, 1 \mathrm{H}, \mathrm{H}-\mathrm{N}(3)), 7.83(\mathrm{~d}, J=1.1 \mathrm{~Hz}, 1 \mathrm{H}, \mathrm{H}-\mathrm{C}(6))$, 7.58 - 7.52 (m, 2H, H-arom), 7.48 - 7.41 (m, 4H, H-arom), 7.28 (t, J=7.7 Hz, 2H, H-arom), 7.21 (t, J = 7.2 Hz, 1H, H-arom), 6.84 (dd, J $=8.9,1.2 \mathrm{~Hz}, 4 \mathrm{H}, \mathrm{H}$-arom), $5.91\left(\mathrm{dd}, J=8.0,5.5 \mathrm{~Hz}, 1 \mathrm{H}, \mathrm{H}-\mathrm{C}\left(1^{\prime}\right)\right), 4.25\left(\mathrm{dt}, J=10.8,6.0 \mathrm{~Hz}, 1 \mathrm{H}, \mathrm{H}-\mathrm{C}\left(5^{\prime}\right)\right), 4.13-4.08\left(\mathrm{~m}, 1 \mathrm{H}, \mathrm{H}-\mathrm{C}\left(4^{\prime}\right)\right)$, $3.86\left(\mathrm{~d}, J=3.4 \mathrm{~Hz}, 1 \mathrm{H}, \mathrm{H}-\mathrm{C}\left(7^{\prime}\right), 3.79\right.$ (s, 6H, MeO), 2.70 (ddd, $J=12.8,10.2,5.5 \mathrm{~Hz}, 1 \mathrm{H}, \mathrm{H}-\mathrm{C}\left(2^{\prime}\right)$ ), 2.61 (dd, $J=16.9,8.2 \mathrm{~Hz}, 1 \mathrm{H}, \mathrm{H}-$ $\left.\mathrm{C}\left(3^{\prime}\right)\right), 1.84(\mathrm{~d}, J=0.8 \mathrm{~Hz}, 3 \mathrm{H}, \mathrm{Me}-\mathrm{C}(5)), 1.80(\mathrm{br}, 1 \mathrm{H}, \mathrm{OH}), 1.60$ (ddd, $\left.J=14.2,10.5,4.2 \mathrm{~Hz}, 1 \mathrm{H}, \mathrm{H}-\mathrm{C}\left(6^{\prime}\right)\right), 1.33$ (dt, $J=12.9,8.0 \mathrm{~Hz}, 1 \mathrm{H}$, $\left.\mathrm{H}-\mathrm{C}\left(2^{\prime}\right)\right), 1.14\left(\mathrm{dd}, J=13.7,6.1 \mathrm{~Hz}, 1 \mathrm{H}, \mathrm{H}-\mathrm{C}\left(6^{\prime}\right)\right) ;{ }^{13} \mathrm{C} \mathrm{NMR}\left(101 \mathrm{MHz}, \mathrm{CDCl}_{3}\right) \delta 164.17$ (C(4)), 158.64 (MeO-C-arom), 150.47 (C(2)), 145.65, 136.85, 136.71 (C-arom), 135.52 (C(6)), 130.20, 128.12, 127.91, 126.90, 113.22, 113.21 (CH-arom), 110.69 (C(5)), 87.21 ( $\left.(\mathrm{Ph})_{3}\right), 86.57\left(\mathrm{C}\left(1^{\prime}\right)\right), 82.02\left(\mathrm{C}\left(4^{\prime}\right)\right), 74.19\left(\mathrm{C}\left(5^{\prime}\right)\right), 74.13\left(\mathrm{C}\left(7^{\prime}\right)\right), 55.25$ (MeO-DMTr), $49.40\left(\mathrm{C}\left(3^{\prime}\right)\right), 38.51\left(\mathrm{C}\left(6^{\prime}\right)\right), 37.64\left(\mathrm{C}\left(2^{\prime}\right)\right), 12.58$ $(\mathrm{Me}-\mathrm{C}(5))$; $\mathrm{ESI}{ }^{+}-\mathrm{HRMS} \mathrm{m} / z$ calcd for $\mathrm{C}_{33} \mathrm{H}_{34} \mathrm{O}_{7} \mathrm{~N}_{2} \mathrm{Na}\left([\mathrm{M}+\mathrm{Na}]^{+}\right)$593.2258, found 593.2250.

$\left(3^{\prime} R, 5^{\prime} R, 7^{\prime} R\right)-1-\left\{7^{\prime}-O-[(2-c y a n o e t h o x y)\right.$-diisopropylaminophosphanyl]-2', 3'-Dideoxy-3', 5'-ethano-5'-O-[(4,4'-dimethoxytriphenyl)methyl]- $\beta$ D-ribofuranosyl\} thymine (12): To a solution of the nucleoside 11 (232 mg, $0.406 \mathrm{mmol})$ and 5-(ethylthio)-1H-tetrazole $(90 \mathrm{mg}, 0.69$ $\mathrm{mmol})$ in dry DCM $(10 \mathrm{~mL})$ was added dropwise 2-cyanoethyl N,N,N',N'-tetraisopropylphosphordiamidite $(0.26 \mathrm{~mL}, 0.81 \mathrm{mmol})$ at rt. After stirring for $30 \mathrm{~min}$, the reaction mixture was diluted with $\mathrm{DCM}(50 \mathrm{~mL})$ and washed with satd $\mathrm{NaHCO}_{3}(2 \mathrm{X} 30 \mathrm{~mL})$ and satd $\mathrm{NaCl}(30$ $\mathrm{mL})$. Aqueous phases were combined and extracted with $\mathrm{DCM}(50 \mathrm{~mL})$. The combined organic phases were dried over $\mathrm{MgSO}_{4}$, filtered and evaporated. The crude product was purified by $\mathrm{CC}\left(1.8 \% \mathrm{MeOH}\right.$ in $\left.\mathrm{DCM},+0.5 \% \mathrm{Et}{ }_{3} \mathrm{~N}\right)$ to yield $12(219 \mathrm{mg}$, mixture of two isomers, $70 \%)$ as a white foam. Data for 12: $R_{\mathrm{f}}=0.68\left(6 \% \mathrm{MeOH}\right.$ in DCM); ${ }^{1} \mathrm{H} \mathrm{NMR}\left(300 \mathrm{MHz}, \mathrm{CDCl}_{3}\right) \delta 8.93(\mathrm{br}, 1 \mathrm{H}, \mathrm{H}-\mathrm{N}(3)), 7.85(\mathrm{~d}, J=1.2$ $\mathrm{Hz}, 1 \mathrm{H}, \mathrm{H}-\mathrm{C}(6)), 7.65$ - 7.52 (m, 2H, H-arom), 7.52 - 7.40 (m, 4H, H-arom), 7.40 - 7.21 (m, 3H, H-arom), 6.96 - 6.81 (m, 4H, H-arom), 6.00, $5.94\left(2 \mathrm{dd}, J=8.3,5.2 \mathrm{~Hz}, 1 \mathrm{H}, \mathrm{H}-\mathrm{C}\left(1^{\prime}\right)\right), 4.29-4.17\left(\mathrm{~m}, 1 \mathrm{H}, \mathrm{H}-\mathrm{C}\left(5^{\prime}\right)\right), 4.12-3.89$ (m, 2H, H-C(4'), H-C(7')), 3.85, $3.84(2 \mathrm{~s}, 6 \mathrm{H}$, $\mathrm{MeO}$ ), 3.81 - $3.63\left(\mathrm{~m}, 2 \mathrm{H}, \mathrm{OCH}_{2} \mathrm{CH}_{2} \mathrm{CN}\right), 3.56-3.41\left(\mathrm{~m}, 2 \mathrm{H},\left(\mathrm{Me}_{2} \mathrm{CH}\right)_{2} \mathrm{~N}\right), 2.88-2.69\left(\mathrm{~m}, 2 \mathrm{H}, \mathrm{H}-\mathrm{C}\left(3^{\prime}\right), \mathrm{H}-\mathrm{C}\left(2^{\prime}\right)\right), 2.61,2.56(\mathrm{dt}, J=12,9$ $\left.6.3 \mathrm{~Hz}, 2 \mathrm{H}, \mathrm{OCH}_{2} \mathrm{CH}_{2} \mathrm{CN}\right), 1.92,1.82(2 \mathrm{~d}, J=0.8 \mathrm{~Hz}, 3 \mathrm{H}, \mathrm{Me}-\mathrm{C}(5)), 1.75-1.56\left(\mathrm{~m}, 1 \mathrm{H}, \mathrm{H}-\mathrm{C}\left(6^{\prime}\right)\right), 1.52-1.36\left(\mathrm{~m}, 2 \mathrm{H}, \mathrm{H}-\mathrm{C}\left(6^{\prime}\right), \mathrm{H}-\mathrm{C}\left(2^{\prime}\right)\right)$, $1.22-1.01\left(\mathrm{~m}, 12 \mathrm{H},\left(\mathrm{Me}_{2} \mathrm{CH}\right)_{2} \mathrm{~N}\right) ;{ }^{13} \mathrm{C}$ NMR $\left(101 \mathrm{MHz}, \mathrm{CDCl}_{3}\right) \delta 163.86(\mathrm{C}(4)), 158.66,158.64$ (MeO-C-arom), 150.29, 150.27 (C(2)), 145.58, 145.52, 136.76, 136.71, 136.69, 136.60 (C-arom), 135.49, 135.35 (C(6)), 130.21, 130.16, 128.17, 128.13, 127.88, 126.91, 126.89 (CH-arom), $117.49\left(\mathrm{OCH}_{2} \mathrm{CH}_{2} \mathrm{CN}\right), 113.18$ ( $\mathrm{CH}$-arom), $110.74(\mathrm{C}(5)), 87.27,87.25\left(\mathrm{C}(\mathrm{Ph})_{3}\right), 86.58,86.45\left(\mathrm{C}\left(1^{\prime}\right)\right), 81.79,81.68$ $\left(\mathrm{C}\left(4^{\prime}\right)\right), 76.02,75.50$ ( JC,P = 16.5, $\left.15.7 \mathrm{~Hz}, \mathrm{C}\left(7^{\prime}\right)\right), 74.22\left(\mathrm{C}\left(5^{\prime}\right)\right)$, 58.26, 58.06, $57.87\left(\mathrm{OCH}_{2} \mathrm{CH}_{2} \mathrm{CN}\right)$, 55.26, 55.22 (MeO-DMTr), 48.85, $48.62\left(J_{C, P}=2.6,5.0 \mathrm{~Hz}, \mathrm{C}\left(3^{\prime}\right)\right), 43.10,43.04\left(J_{C, P}=12.3,12.4 \mathrm{~Hz}\left(\mathrm{Me}_{2} \mathrm{CH}\right)_{2} \mathrm{~N}\right), 37.78\left(J_{C, P}=5.3 \mathrm{~Hz} C\left(6^{\prime}\right)\right), 37.62,37.48\left(\mathrm{C}\left(2^{\prime}\right)\right), 37.41$ $\left.\left(J_{C, P}=3.6 \mathrm{~Hz} \mathrm{C}\left(6^{\prime}\right)\right), 24.57,24.53,24.50,24.46,24.44,24.39,24.37\left(M e_{2} \mathrm{CH}\right)_{2} \mathrm{~N}\right), 20.35,20.25\left(J_{\mathrm{C}, \mathrm{P}}=7.1,7.0 \mathrm{~Hz}, \mathrm{OCH}_{2} \mathrm{CH}_{2} \mathrm{CN}\right)$, 12.58, $12.41(7 \mathrm{~s}, \mathrm{Me}-\mathrm{C}(5)) ;{ }^{31} \mathrm{P} \mathrm{NMR}\left(122 \mathrm{MHz}, \mathrm{CDCl}_{3}\right) \delta 147.32,146.98 ; \mathrm{ESI}{ }^{+}-\mathrm{HRMS} \mathrm{m} / z$ calcd for $\mathrm{C}_{42} \mathrm{H}_{52} \mathrm{O}_{8} \mathrm{~N}_{4} \mathrm{P}\left(\left[\mathrm{M}+\mathrm{H}^{+}\right) 771.3517\right.$, found 771.3512 
(3'S, 5'R, 7'R)-N4-Benzoyl-1-\{2',3'-Dideoxy-3', 5'-ethano-7'-hydroxy-5'-O-[(4,4'-dimethoxytriphenyl)methyl]- $\beta$-D-ribofuranosyl\}-5methylcytosine (13): To a solution of the nucleoside 11 (302 mg, $0.530 \mathrm{mmol})$ in dry MeCN (5 mL) was added dropwise BSA (0.31 mL, $1.27 \mathrm{mmol}$ ) at $0^{\circ}$, and then the solution was stirred overnight at $\mathrm{rt}$. In another flask, a suspension of 1,2,4-triazole (1.28 $\left.\mathrm{g}, 18.55 \mathrm{mmol}\right)$ in dry $\mathrm{MeCN}(50 \mathrm{~mL})$ was cooled down to $0^{\circ} \mathrm{C}$ and $\mathrm{POCl}_{3}(0.40 \mathrm{~mL}, 4.2 \mathrm{mmol})$ and $\mathrm{Et}_{3} \mathrm{~N}(2.96 \mathrm{~mL}, 21.2 \mathrm{mmol})$ were added. The suspension was stirred for $30 \mathrm{~min}$ at $0^{\circ} \mathrm{C}$, and then the previous prepared solution of the silylated compound $\mathbf{1 1}$ was added to the suspension and the mixture was further stirred for $5 \mathrm{~h}$ at rt. Reaction was quenched with addition of satd $\mathrm{NaHCO}_{3}(10 \mathrm{~mL}), \mathrm{MeCN}$ removed under reduced pressure and the resulting mixture diluted with satd $\mathrm{NaHCO}_{3}(35 \mathrm{~mL})$ and extracted with $\mathrm{DCM}(3 \mathrm{X} 40 \mathrm{~mL})$. The combined organic phases were dried over $\mathrm{MgSO}_{4}$, filtered and evaporated. The crude product was then dissolved in a mixture of 1,4dioxane $(10 \mathrm{~mL})$ and concd $\mathrm{NH}_{4} \mathrm{OH}(10 \mathrm{~mL})$. After stirring for $2 \mathrm{~h}$ at $\mathrm{rt}$, the mixture was reduced to half of its volume in vacuo, diluted with satd $\mathrm{NaHCO}_{3}(30 \mathrm{~mL})$ and extracted with $\mathrm{DCM}(4 \times 30 \mathrm{~mL})$. The combined organic phases were dried over $\mathrm{MgSO}_{4}$, filtered and evaporated. The crude product was then dissolved in dry DMF (13 mL), $\mathrm{Et}_{3} \mathrm{~N}(90 \mu \mathrm{L}, 0.64 \mathrm{mmol})$ followed by Bz2 $\mathrm{O}(300 \mathrm{mg}, 1.33 \mathrm{mmol})$ were added at rt and the solution was stirred overnight. The resulting brown solution was quenched by careful addition of satd $\mathrm{NaHCO}_{3}$ $(50 \mathrm{~mL})$ and extracted with $\mathrm{DCM}(4 \times 50 \mathrm{~mL})$. The combined organic phases were dried over $\mathrm{MgSO}_{4}$, filtered and evaporated. The crude product was purified by CC (hexane/EtOAc 1:2, $+0.5 \% \mathrm{Et}_{3} \mathrm{~N}$ ) to yield $13(315 \mathrm{mg}, 88 \%)$ as a white foam. Data for 13: $R_{\mathrm{f}}=0.57(4 \%$ $\mathrm{MeOH}$ in DCM); ${ }^{1} \mathrm{H}$ NMR (300 MHz, $\left.\mathrm{CDCl}_{3}\right) \delta 13.39$ (br, 1H, NH), $8.46-8.26(\mathrm{~m}, 2 \mathrm{H}, \mathrm{H}$-arom), 8.13 (d, $J=0.5 \mathrm{~Hz}, 1 \mathrm{H}, \mathrm{C}(6)), 7.61$ (d, $J$ $=7.3 \mathrm{~Hz}, 2 \mathrm{H}, \mathrm{H}$-arom), $7.58-7.43$ (m, 7H, H-arom), 7.34 (t, $J=7.4 \mathrm{~Hz}, 2 \mathrm{H}, \mathrm{H}$-arom), $7.30-7.23$ (m, 1H, H-arom), 6.89 (d, $J=8.8 \mathrm{~Hz}$, $4 \mathrm{H}, \mathrm{H}$-arom), 5.96 (dd, J=7.5, $\left.5.8 \mathrm{~Hz}, 1 \mathrm{H}, \mathrm{H}-\mathrm{C}\left(1^{\prime}\right)\right), 4.38-4.25\left(\mathrm{~m}, 1 \mathrm{H}, \mathrm{H}-\mathrm{C}\left(5^{\prime}\right)\right), 4.22-4.12\left(\mathrm{~m}, 1 \mathrm{H}, \mathrm{H}-\mathrm{C}\left(4^{\prime}\right)\right), 3.90(\mathrm{~d}, J=3.6 \mathrm{~Hz}, 1 \mathrm{H}$, $\left.\mathrm{H}-\mathrm{C}\left(7^{\prime}\right)\right), 3.83$ (s, 6H, MeO), 2.82 (ddd, $\left.J=13.3,10.2,5.7 \mathrm{~Hz}, 1 \mathrm{H}, \mathrm{H}-\mathrm{C}\left(2^{\prime}\right)\right), 2.66$ (dd, $\left.J=17.0,8.1 \mathrm{~Hz}, 1 \mathrm{H}, \mathrm{H}-\mathrm{C}\left(3^{\prime}\right)\right), 2.08(\mathrm{~s}, 3 \mathrm{H}, M e-$ $\mathrm{C}(5)), 1.77(b r, 1 \mathrm{H}, \mathrm{OH}), 1.71-1.57\left(\mathrm{~m}, 1 \mathrm{H}, \mathrm{H}-\mathrm{C}\left(6^{\prime}\right)\right), 1.49-1.36\left(\mathrm{~m}, 1 \mathrm{H}, \mathrm{H}-\mathrm{C}\left(2^{\prime}\right)\right), 1.21\left(\mathrm{dd}, J=13.7,6.2 \mathrm{~Hz}, 1 \mathrm{H}, \mathrm{H}-\mathrm{C}\left(6^{\prime}\right)\right) ;{ }^{13} \mathrm{C}$ NMR $(75$ $\mathrm{MHz}, \mathrm{CDCl}_{3}$ ) $\delta 179.56(\mathrm{CONH}), 160.01$ (C(4)), 158.70 (MeO-C-arom), 147.96 (C(2)), 145.65 (C-arom), 137.26 (C(6)), 136.99, 136.83, 136.71 (C-arom), 132.41, 130.22, 129.89, 128.16, 128.14, 127.95, 126.94, 113.25 (CH-arom), $111.57(\mathrm{C}(5)), 87.34(C(\mathrm{Ph}) 3), 87.32$

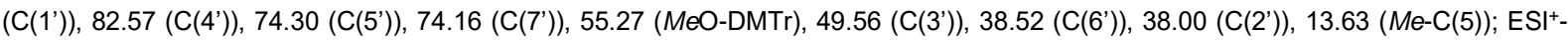
HRMS $m / z$ calcd for $\mathrm{C}_{40} \mathrm{H}_{40} \mathrm{O}_{7} \mathrm{~N}_{3}\left([\mathrm{M}+\mathrm{H}]^{+}\right) 674.2861$, found 674.2862 .

$\left(3^{\prime} R, 5^{\prime} R, 7^{\prime} R\right)-N 4-B e n z o y l-1-\left\{7^{\prime}-O-[(2-c y a n o e t h o x y)\right.$-diisopropylaminophosphanyl]-2', 3'-Dideoxy-3', 5'-ethano-5'-O-[(4,4'-

dimethoxytriphenyl)methyl]- $\beta$-D-ribofuranosyl\}-5-methylcytosine (14): To a solution of the nucleoside 13 (276 mg, $0.409 \mathrm{mmol})$ and 5(ethylthio)-1H-tetrazole $(69 \mathrm{mg}, \quad 0.53 \mathrm{mmol})$ in dry $\mathrm{DCM}(10 \mathrm{~mL})$ was added dropwise 2-cyanoethyl N,N, $\mathrm{N}^{\prime}, \mathrm{N}^{\prime}$ tetraisopropylphosphordiamidite $(0.20 \mathrm{~mL}, 0.61 \mathrm{mmol})$ at $\mathrm{rt}$. After stirring for $60 \mathrm{~min}$, the reaction mixture was diluted with $\mathrm{DCM}(50 \mathrm{~mL})$ and washed with satd $\mathrm{NaHCO}_{3}(2 \times 30 \mathrm{~mL})$ and satd $\mathrm{NaCl}(30 \mathrm{~mL})$. Aqueous phases were combined and extracted with $\mathrm{DCM}(50 \mathrm{~mL})$. The combined organic phases were dried over $\mathrm{MgSO}_{4}$, filtered and evaporated. The crude product was purified by CC (EtOAc/hexanne $\left.2: 3,+0.5 \% \mathrm{Et}_{3} \mathrm{~N}\right)$ to yield $14(268 \mathrm{mg}$, mixture of two isomers, $75 \%)$ as a white foam. Data for $14: R_{\mathrm{f}}=0.77(5 \% \mathrm{MeOH}$ in $\mathrm{DCM}) ;{ }^{1} \mathrm{H}$ NMR (400 MHz, $\left.\mathrm{CDCl}_{3}\right) \delta 13.32(\mathrm{~s}, 1 \mathrm{H}, \mathrm{NH}), 8.41-8.28$ (m, 2H, H-arom), $8.13-8.04(\mathrm{~m}, 1 \mathrm{H}, \mathrm{C}(6)), 7.61-7.51$ (m, 3H, H-arom), 7.51 - 7.40 (m, 6H, H-arom), 7.37 - 7.29 (m, 2H, H-arom), 7.29 - 7.20 (m, 1H, H-arom), 6.92 - 6.82 (m, 4H, H-arom), 6.07 - 5.87 (m, $1 \mathrm{H}, \mathrm{H}$ $\left.\mathrm{C}\left(1^{\prime}\right)\right), 4.24$ (dq, $\left.J=11.7,5.8 \mathrm{~Hz}, 1 \mathrm{H}, \mathrm{H}-\mathrm{C}\left(5^{\prime}\right)\right), 4.13-4.00$ (m, $\left.1 \mathrm{H}, \mathrm{H}-\mathrm{C}\left(4^{\prime}\right)\right), 3.94$ (ddd, $\left.J=14.5,10.5,2.8 \mathrm{~Hz}, 1 \mathrm{H}, \mathrm{H}-\mathrm{C}\left(7^{\prime}\right)\right), 3.83,3.82$ (2s, 6H, MeO), $3.69\left(\mathrm{~m}, 2 \mathrm{H}, \mathrm{OCH}_{2} \mathrm{CH}_{2} \mathrm{CN}\right), 3.53-3.40\left(\mathrm{~m}, 2 \mathrm{H},\left(\mathrm{Me}_{2} \mathrm{CH}\right)_{2} \mathrm{~N}\right), 2.91-2.70\left(\mathrm{~m}, 2 \mathrm{H}, \mathrm{H}-\mathrm{C}\left(2^{\prime}\right), \mathrm{H}-\mathrm{C}\left(3^{\prime}\right)\right), 2.57,2.53(2 \mathrm{t}, J=6.3$ $\left.\mathrm{Hz}, 2 \mathrm{H}, \mathrm{OCH}_{2} \mathrm{CH}_{2} \mathrm{CN}\right), 2.08,1.99(2 \mathrm{~d}, J=0.6 \mathrm{~Hz}, 3 \mathrm{H}, \mathrm{Me}-\mathrm{C}(5)), 1.72-1.56\left(\mathrm{~m}, 1 \mathrm{H}, \mathrm{H}-\mathrm{C}\left(6^{\prime}\right)\right), 1.54-1.36\left(\mathrm{~m}, 2 \mathrm{H}, \mathrm{H}-\mathrm{C}\left(2^{\prime}\right), \mathrm{H}-\mathrm{C}\left(6^{\prime}\right)\right), 1.10$ $\left(\mathrm{m}, 12 \mathrm{H},\left(\mathrm{Me}_{2} \mathrm{CH}\right)_{2} \mathrm{~N}\right) ;{ }^{13} \mathrm{C} \mathrm{NMR}\left(101 \mathrm{MHz}, \mathrm{CDCl}_{3}\right) \delta 179.54(\mathrm{CONH}), 159.98$ (C(4)), 158.69 (MeO-C-arom), 147.90 (C(2)), 145.58, 145.54 (C-arom), 137.30, 136.93 (C(6)), 136.81, 136.80, 136.73, 136.70, 136.67, 136.60 (C-arom), 132.37, 132.35, 130.22, 130.17, 129.89, 128.17, 128.15, 128.11, 127.93, 126.94 (CH-arom), $117.49\left(\mathrm{OCH}_{2} \mathrm{CH}_{2} \mathrm{CN}\right), 113.23(\mathrm{CH}$-arom), $111.60(\mathrm{C}(5)), 87.36,87.35$ $(C(\mathrm{Ph}) 3), 87.33,87.25\left(\mathrm{C}\left(1^{\prime}\right)\right), 82.33,82.25\left(\mathrm{C}\left(4^{\prime}\right)\right), 76.05,75.52$ ( JC,P = 16.4, 15.6 Hz, C(7')), $74.32\left(\mathrm{C}\left(5^{\prime}\right)\right), 58.18,57.98(J \mathrm{C}, \mathrm{P}=19.5 \mathrm{~Hz}$ $\mathrm{OCH}_{2} \mathrm{CH}_{2} \mathrm{CN}$ ), 55.28, 55.24 (MeO-DMTr), 48.93, $48.72\left(\mathrm{JC}, \mathrm{P}=2.7,4.9 \mathrm{~Hz}, \mathrm{C}\left(3^{\prime}\right)\right), 43.11,43.05\left(\mathrm{JC}, \mathrm{P}=12.4 \mathrm{~Hz}\left(\mathrm{Me}_{2} \mathrm{CH}\right)_{2} \mathrm{~N}\right), 38.02$, $\left.37.88\left(\mathrm{C}\left(2^{\prime}\right)\right), 37.74,37.40\left(\mathrm{JC}, \mathrm{P}=5.3,3.4 \mathrm{~Hz}, \mathrm{C}\left(6^{\prime}\right)\right), 24.58,24.54,24.50,24.47,24.40,24.38\left(6 \mathrm{~s}, \mathrm{Me}_{2} \mathrm{CH}\right) 2 \mathrm{~N}\right), 20.36,20.26\left(J_{\mathrm{C}, \mathrm{P}}=7.1\right.$ 
$\mathrm{Hz}, \mathrm{OCH}_{2} \mathrm{CH}_{2} \mathrm{CN}$ ), ), 13.66, $13.49(\mathrm{Me}-\mathrm{C}(5)) ;{ }^{31} \mathrm{P} \mathrm{NMR}\left(122 \mathrm{MHz}, \mathrm{CDCl}_{3}\right) \delta 147.37,147.07$; ESI+-HRMS m/z calcd for $\mathrm{C}_{49} \mathrm{H}_{57} \mathrm{O}_{8} \mathrm{~N}_{5} \mathrm{P}([\mathrm{M}+$ H]+ $]^{+}$874.3939, found 874.3937 .

(3aR,4R,6R,6aS)-4-((tert-butyldiphenylsilyl)oxy)-2-methoxyhexahydro-2H-cyclopenta[b]furan-6-yl acetate (7): To a solution of sugar 6 $(3.35 \mathrm{~g}, 8.12 \mathrm{mmol})$ and 4-dimethylaminopyridine $(1.29 \mathrm{~g}, 10.6 \mathrm{mmol})$ in dry DCM $(100 \mathrm{~mL})$ was added acetic anhydride $(3.8 \mathrm{~mL}, 41$ $\mathrm{mmol}$ ) at rt. After stirring for $2 \mathrm{~h}$, reaction was quenched by slow addition of satd $\mathrm{NaHCO}_{3}(10 \mathrm{~mL})$. The mixture was then diluted with satd $\mathrm{NaHCO}_{3}(50 \mathrm{~mL})$ and extracted with $\mathrm{DCM}(3 \times 50 \mathrm{~mL})$. The combined organic phases were dried over $\mathrm{MgSO}_{4}$, filtered and evaporated. The crude product was purified by CC (EtOAc/Hexanne 1:2) to yield a mixture of $7(3.53 \mathrm{~g}, 96 \%)$ in an anomeric ratio $\alpha / \beta \approx$ 4:1 as a colorless oil. Data for 7: $R_{\mathrm{f}}=0.42\left(\mathrm{EtOAc} /\right.$ hexane 1:2); ${ }^{1} \mathrm{H} \mathrm{NMR}\left(400 \mathrm{MHz}, \mathrm{CDCl}_{3}\right) \delta 7.70-7.59(\mathrm{~m}, 4 \mathrm{H}, \mathrm{H}$-arom), $7.48-7.34$ (m, 6H, H-arom), 5.41 (dt, $J=11.0,5.6 \mathrm{~Hz}, 0.8 \mathrm{H}, \mathrm{H}-\mathrm{C}(6)), 5.28$ (ddd, $J=11.7,6.6,5.2 \mathrm{~Hz}, 0.2 \mathrm{H}, \mathrm{H}-\mathrm{C}(6)), 4.99(\mathrm{~d}, J=4.8 \mathrm{~Hz}, 0.8 \mathrm{H}, \mathrm{H}$ $\mathrm{C}(2)), 4.89$ - 4.81(m, 0.4H, H-C(2), H-C(6a)), $4.76-4.69$ (m, 0.8H, H-C(6a)), 4.11 (d, J = 5.1 Hz, 0.2H, H-C(4)), 3.90 (d, J = 4.0 Hz, 0.8H, H-C(4)), 3.27, 3.24 (2s, 3H, MeO), 2.81 (dd, $J=16.6,7.6 \mathrm{~Hz}, 0.8 \mathrm{H}, \mathrm{H}-\mathrm{C}(3 \mathrm{a})$ ), 2.60 (dd, $J=10.1,7.0 \mathrm{~Hz}, 0.2 \mathrm{H}, \mathrm{H}-\mathrm{C}(3 \mathrm{a})$ ), $2.30-$ $2.18(\mathrm{~m}, 0.2 \mathrm{H}, \mathrm{H}-\mathrm{C}(5)), 2.12,2.10\left(2 \mathrm{~s}, J=4.7 \mathrm{~Hz}, 3 \mathrm{H}, \mathrm{MeCO}_{2}\right.$ ), $2.07-1.82(\mathrm{~m}, 2.8 \mathrm{H}, \mathrm{H}-\mathrm{C}(5), \mathrm{H}-\mathrm{C}(3)), 1.24$ (ddd, $J=12.9,7.6,3.7 \mathrm{~Hz}$, $1 \mathrm{H}, \mathrm{H}-\mathrm{C}(3)), 1.07$ (s, 9H, (CH3)3-C-Si); ${ }^{13} \mathrm{C} \mathrm{NMR}\left(75 \mathrm{MHz}, \mathrm{CDCl}_{3}\right) \delta$ 170.75, $170.66\left(\mathrm{MeCO}_{2}\right), 135.77,135.73,135.72$ (CH-arom), 133.75, 133.65 (C-arom), 129.82, 129.74, 127.76, 127.75, 127.71 (CH-arom), 106.19, 106.15 (C(2)), 83.17, 79.80 (C(6a)), $77.49,76.46$ (C(4)), 75.64, $74.41(\mathrm{C}(6))$, 54.34, $54.25(\mathrm{MeO}), 51.48,50.17$ (C(3a)), 38.05, $37.98(\mathrm{C}(3)), 36.96,36.21(\mathrm{C}(5)), 26.95,26.90\left(\mathrm{CH}_{3}\right)_{3}-\mathrm{C}-$ $\mathrm{Si})$, 21.09, $\left.21.04\left(\mathrm{MeCO}_{2}\right), 19.04\left(\mathrm{CH}_{3}\right)_{3}-\mathrm{C}-\mathrm{Si}\right)$; $\mathrm{ESI}{ }^{+}-\mathrm{HRMS} \mathrm{m} / \mathrm{z}$ calcd for $\mathrm{C}_{26} \mathrm{H}_{34} \mathrm{O}_{5} \mathrm{NaSi}\left([\mathrm{M}+\mathrm{Na}]^{+}\right)$477.2068, found 477.2063.

(3'R, 5'R, 7'R)-N6-Benzoyl-9-\{5'-O-acetyl-7'-[(tert-butyldiphenylsilyl)oxy]-2', 3'-Dideoxy-3', 5'-ethano- $\alpha, \beta-D$-ribofuranosyl\} adenine (15): To a suspension of sugar $7(1.86 \mathrm{~g}, 4.10 \mathrm{mmol})$ and $\mathrm{N}^{6}$-benzoyladenine $(1.96 \mathrm{~g}, 8.20 \mathrm{mmol})$ in dry MeCN $(40 \mathrm{~mL})$ was added BSA $(4.00 \mathrm{~mL}$, $16.4 \mathrm{mmol})$ at rt. After stirring for $25 \mathrm{~min}$, the suspension became a clear solution and then was heated to $70^{\circ} \mathrm{C}$. TMSOTf $(1.48 \mathrm{~mL}, 8.20$ $\mathrm{mmol}$ ) was added dropwise and the solution was further stirred for $20 \mathrm{~min}$ at $70^{\circ} \mathrm{C}$. The solution was then cooled down to rt, quenched with addition of satd $\mathrm{NaHCO}_{3}(100 \mathrm{~mL})$ and extracted with EtOAc $(4 \mathrm{X} 50 \mathrm{~mL})$. The combined organic phases were dried over $\mathrm{MgSO}_{4}$, filtered and evaporated. The crude product was purified by CC (2\% MeOH in DCM) to yield a mixture of 15 (1.74 g, 64\%) in an anomeric ratio $\alpha / \beta \approx 4: 1$ as a white foam. Data for $15: R_{\mathrm{f}}=0.33$ (EtOAc/hexane 4:1); ${ }^{1} \mathrm{H} \mathrm{NMR}\left(400 \mathrm{MHz}, \mathrm{CDCl}_{3}\right) \delta 9.33(b r, 1 \mathrm{H}, \mathrm{NH}), 8.68(\mathrm{~d}, J=$ $5.4 \mathrm{~Hz}, 0.8 \mathrm{H}, \mathrm{H}-\mathrm{C}(2)), 8.64$ (d, $J=5.6 \mathrm{~Hz}, 0.2 \mathrm{H}, \mathrm{H}-\mathrm{C}(2)), 8.10$ (d, $J=1.5 \mathrm{~Hz}, 0.2 \mathrm{H}$. H-C(8)), 7.99 (d, J=7.3 Hz, 2H, H-arom), 7.95 (s, $0.8 \mathrm{H}, \mathrm{H}-\mathrm{C}(8)), 7.63$ (t, $J=8.7 \mathrm{~Hz}, 4 \mathrm{H}, \mathrm{H}$-arom), 7.55 (dd, $J=13.0,6.4 \mathrm{~Hz}, 1 \mathrm{H}, \mathrm{H}$-arom), $7.50-7.34$ (m, 8H, H-arom), 6.20 (dd, $J=6.3$, $\left.2.5 \mathrm{~Hz}, 0.8 \mathrm{H}, \mathrm{H}-\mathrm{C}\left(1^{\prime}\right)\right), 6.05\left(\mathrm{t}, J=6.5 \mathrm{~Hz}, 0.2 \mathrm{H}, \mathrm{H}-\mathrm{C}\left(1^{\prime}\right)\right), 5.43-5.32\left(\mathrm{~m}, 1 \mathrm{H}, \mathrm{H}-\mathrm{C}\left(5^{\prime}\right)\right), 5.03-4.97\left(\mathrm{~m}, 0.8 \mathrm{H}, \mathrm{H}-\mathrm{C}\left(4^{\prime}\right)\right), 4.83(\mathrm{t}, J=6.0 \mathrm{~Hz}$, $\left.0.2 \mathrm{H}, \mathrm{H}-\mathrm{C}\left(4^{\prime}\right)\right), 4.14\left(b r, 0.2 \mathrm{H}, \mathrm{H}-\mathrm{C}\left(7^{\prime}\right)\right), 4.08\left(\mathrm{~d}, J=3.7 \mathrm{~Hz}, 0.8 \mathrm{H}, \mathrm{H}-\mathrm{C}\left(7^{\prime}\right)\right), 3.02\left(\mathrm{dd}, J=16.1,6.6 \mathrm{~Hz}, 0.8 \mathrm{H}, \mathrm{H}-\mathrm{C}\left(3^{\prime}\right)\right), 2.83(\mathrm{dd}, J=16.9$, $\left.7.7 \mathrm{~Hz}, 0.2 \mathrm{H}, \mathrm{H}-\mathrm{C}\left(3^{\prime}\right)\right), 2.59-2.39\left(\mathrm{~m}, 1 \mathrm{H}, \mathrm{H}-\mathrm{C}\left(2^{\prime}\right)\right), 2.18-2.11\left(\mathrm{~m}, 1 \mathrm{H}, \mathrm{H}-\mathrm{C}\left(6^{\prime}\right)\right), 2.07$ (d, J=1.6 Hz, 2.4H, MeCO $), 2.02(\mathrm{~d}, J=1.9 \mathrm{~Hz}$, 0.6H, MeCO 2$), 2.01-1.92\left(\mathrm{~m}, 1 \mathrm{H}, \mathrm{H}-\mathrm{C}\left(6^{\prime}\right)\right), 1.91-1.80\left(\mathrm{~m}, 1 \mathrm{H}, \mathrm{H}-\mathrm{C}\left(3^{\prime}\right)\right), 1.07$ (s, 9H, $\left.\left(\mathrm{CH}_{3}\right)_{3}-\mathrm{C}-\mathrm{Si}\right) ;{ }^{13} \mathrm{C} \mathrm{NMR}\left(101 \mathrm{MHz}, \mathrm{CDCl}_{3}\right) \delta$ 170.57, $170.49\left(\mathrm{MeCO}_{2}\right), 164.82(\mathrm{CONH}), 152.50(\mathrm{C}(2)), 151.27(\mathrm{C}(4)), 149.56(\mathrm{C}(6)), 141.37,141.06(\mathrm{C}(8)), 135.72,135.68,135.66$ (CH-arom), 133.67, 133.57, 133.24, 133.22 (C-arom), 132.73, 130.03, 129.98, 128.80, 128.78, 127.92, 127.86, 127.85 (CH-arom), $123.61(\mathrm{C}(5)), 87.19,86.17\left(\mathrm{C}\left(1^{\prime}\right)\right), 83.22,80.96\left(\mathrm{C}\left(4^{\prime}\right), 76.50,76.04\left(\mathrm{C}\left(7^{\prime}\right)\right), 74.38\left(\mathrm{C}\left(5^{\prime}\right)\right), 51.07\left(\mathrm{C}\left(3^{\prime}\right)\right), 37.29,37.15,36.80,36.60\right.$ (C(2'), C(6')), $\left.\left.26.89\left(\mathrm{CH}_{3}\right)_{3}-\mathrm{C}-\mathrm{Si}\right), 20.97,20.90\left(\mathrm{MeCO}_{2}\right), 19.01\left(\mathrm{CH}_{3}\right)_{3}-\mathrm{C}-\mathrm{Si}\right)$; ESI+ ${ }^{+} \mathrm{HRMS} \mathrm{m} / 2$ calcd for $\mathrm{C}_{37} \mathrm{H}_{40} \mathrm{O}_{5} \mathrm{~N}_{5} \mathrm{Si}\left(\left[\mathrm{M}+\mathrm{H}^{+}\right)\right.$ 662.2793 , found 662.2787 .

(3'R, 5'R,7'R)-N6-Benzoyl-9-\{7'-[(tert-butyldiphenylsilyl)oxy]-2',3'-Dideoxy-3', 5'-ethano- $\beta$-D-ribofuranosyl\} adenine (16): The nucleoside $15(1.74 \mathrm{~g}, 2.64 \mathrm{mmol})$ was dissolved in $0.15 \mathrm{M} \mathrm{NaOH}$ in THF/methanol/ $\mathrm{H}_{2} \mathrm{O}(5: 4: 1,80 \mathrm{~mL})$ at $0^{\circ} \mathrm{C}$. The reaction was stirred for $20 \mathrm{~min}$ and quenched by addition of $\mathrm{NH}_{4} \mathrm{Cl}(1.06 \mathrm{~g})$. Solvents were then removed under reduced pressure and the product purified by $\mathrm{CC}(5 \%$ isopropanol in DCM) to yield $16(287 \mathrm{mg}, 18 \%)$ and its corresponding $\alpha$ anomer $(836 \mathrm{mg}, 51 \%)$ as white foams. Data for 16: $R_{\mathrm{f}}=0.44$ (6\% MeOH in DCM); ${ }^{1} \mathrm{H} \mathrm{NMR} \mathrm{(300} \mathrm{MHz,} \mathrm{CDCl} 3$ ) $\delta 8.70$ (s, 1H, H-C(2)), $8.09-7.98$ (m, 2H, H-arom), 7.97 (s, 1H, H-C(8)), 7.63 (ddd, $J=$ 
7.4, 5.7, $1.5 \mathrm{~Hz}, 4 \mathrm{H}, \mathrm{H}$-arom), 7.59 - 7.55 (m, 1H, H-arom), 7.51 (m, 2H, H-arom), $7.44-7.33$ (m, 6H, H-arom), 6.02 (dd, J = 9.4, 5.5 $\left.\mathrm{Hz}, 1 \mathrm{H}, \mathrm{H}-\mathrm{C}\left(1^{\prime}\right)\right), 4.57\left(\mathrm{dd}, J=8.1,5.0 \mathrm{~Hz}, 1 \mathrm{H}, \mathrm{H}-\mathrm{C}\left(4^{\prime}\right)\right), 4.43\left(\mathrm{dd}, J=11.8,5.3 \mathrm{~Hz}, 1 \mathrm{H}, \mathrm{H}-\mathrm{C}\left(5^{\prime}\right)\right), 4.26\left(b r, 1 \mathrm{H}, \mathrm{H}-\mathrm{C}\left(7^{\prime}\right)\right), 2.78(\mathrm{q}, J=8.9$ $\left.\mathrm{Hz}, 1 \mathrm{H}, \mathrm{H}-\mathrm{C}\left(3^{\prime}\right)\right), 2.32-1.80$ (m, 5H, H-C(2'), H-C(6'), OH), 1.06 (s, 9H, ( $\left.\left.\mathrm{CH}_{3}\right)_{3}-\mathrm{C}-\mathrm{Si}\right) ;{ }^{13} \mathrm{C} \mathrm{NMR}\left(101 \mathrm{MHz}, \mathrm{CDCl}_{3}\right) \delta 164.85(\mathrm{CONH})$, $152.56(\mathrm{C}(2)), 151.17(\mathrm{C}(4)), 149.86(\mathrm{C}(6)), 141.25$ (C(8)), 135.68 (CH-arom), 133.87, 133.39 (C-arom), 132.78, 129.92, 128.78, 128.01, 127.78 (CH-arom), $123.51(\mathrm{C}(5)), 87.65\left(\mathrm{C}\left(1^{\prime}\right)\right), 82.91\left(\mathrm{C}\left(4^{\prime}\right)\right), 76.66$ (C(7')), $72.54\left(\mathrm{C}\left(5^{\prime}\right)\right), 50.44\left(\mathrm{C}\left(3^{\prime}\right)\right), 41.42\left(\mathrm{C}\left(6^{\prime}\right)\right), 36.17\left(\mathrm{C}\left(2^{\prime}\right)\right)$, $\left.26.89\left(\mathrm{CH}_{3}\right)_{3}-\mathrm{C}-\mathrm{Si}\right), 19.03\left(\mathrm{CH}_{3}\right)_{3}-\mathrm{C}$-Si); $\mathrm{ESI}^{+}-\mathrm{HRMS} \mathrm{m} / \mathrm{z}$ calcd for $\mathrm{C}_{35} \mathrm{H}_{38} \mathrm{O}_{4} \mathrm{~N}_{5} \mathrm{Si}\left([\mathrm{M}+\mathrm{H}]^{+}\right) 620.2688$, found 620.2671.

$\left(3^{\prime} R, 5^{\prime} R, 7^{\prime} R\right)-N 6-B e n z o y l-9-\left\{7^{\prime}-[(\right.$ tert-butyldiphenylsilyl)oxy]-2', 3'-Dideoxy-3', 5'-ethano-5'-O-[(4,4'-dimethoxytriphenyl)methyl]- $\beta$-Dribofuranosyl\} adenine (17): To a solution of nucleoside $16(307 \mathrm{mg}, 0.495 \mathrm{mmol})$ in dry pyridine $(6 \mathrm{~mL})$ was added DMTr-Cl (503 mg, $1.49 \mathrm{mmol})$ at rt. The solution was stirred for 1 day and then diluted with satd $\mathrm{NaHCO}_{3}(50 \mathrm{~mL})$ and extracted with $\mathrm{DCM}(3 \times 70 \mathrm{~mL})$. The combined organic phases were dried over $\mathrm{MgSO}_{4}$, filtered and evaporated. The crude product was purified by CC $(1.5 \% \mathrm{MeOH}$ in $\mathrm{DCM},+0.5 \% \mathrm{Et} 3 \mathrm{~N})$ to yield $17(395 \mathrm{mg}, 87 \%)$ as a yellow foam. Data for 17: $R_{\mathrm{f}}=0.65\left(5 \% \mathrm{MeOH}\right.$ in DCM); ${ }^{1} \mathrm{H} \mathrm{NMR}(300 \mathrm{MHz}, \mathrm{MeOD})$ $\delta 8.64(\mathrm{~s}, 1 \mathrm{H}, \mathrm{H}-\mathrm{C}(2)), 8.61$ (s, $1 \mathrm{H}, \mathrm{H}-\mathrm{C}(8)), 8.08(\mathrm{~d}, J=7.2 \mathrm{~Hz}, 2 \mathrm{H}, \mathrm{H}$-arom), $7.68-7.17$ (m, 22H, H-arom), $6.86-6.75(\mathrm{~m}, 4 \mathrm{H}, \mathrm{H}-$ arom), 6.14 (dd, J = 7.4, $\left.6.3 \mathrm{~Hz}, 1 \mathrm{H}, \mathrm{H}-\mathrm{C}\left(1^{\prime}\right)\right), 4.48-4.31\left(\mathrm{~m}, 1 \mathrm{H}, \mathrm{H}-\mathrm{C}\left(5^{\prime}\right)\right), 4.28-4.15\left(\mathrm{~m}, 1 \mathrm{H}, \mathrm{H}-\mathrm{C}\left(4^{\prime}\right)\right), 3.88(\mathrm{~d}, J=3.8 \mathrm{~Hz}, 1 \mathrm{H}, \mathrm{H}-$ $\left.\mathrm{C}\left(7^{\prime}\right)\right)$, 3.75, $3.74(2 \mathrm{~s}, 6 \mathrm{H}, \mathrm{MeO}), 2.67$ (dd, $\left.J=16.6,6.7 \mathrm{~Hz}, 1 \mathrm{H}, \mathrm{H}-\mathrm{C}\left(3^{\prime}\right)\right), 2.47$ (ddd, $\left.J=13.3,10.2,6.1 \mathrm{~Hz}, 1 \mathrm{H}, \mathrm{H}-\mathrm{C}\left(2^{\prime}\right)\right), 2.15-1.94(\mathrm{~m}$, $\left.1 \mathrm{H}, \mathrm{H}-\mathrm{C}\left(6^{\prime}\right)\right), 1.71$ (ddd, $\left.J=13.0,11.3,4.4 \mathrm{~Hz}, 1 \mathrm{H}, \mathrm{H}-\mathrm{C}\left(2^{\prime}\right)\right), 1.11$ (dd, $\left.J=12.2,4.9 \mathrm{~Hz}, 1 \mathrm{H}, \mathrm{H}-\mathrm{C}\left(6^{\prime}\right)\right), 0.95\left(\mathrm{~s}, 9 \mathrm{H},\left(\mathrm{CH}_{3}\right) 3^{-} \mathrm{C}-\mathrm{Si}\right) ;{ }^{13} \mathrm{C} \mathrm{NMR}$ (75 MHz, $\mathrm{CDCl}_{3}$ ) $\delta 164.69$ (CONH), 158.61, 158.60 (MeO-C-arom), 152.42 (C(2)), 151.27 (C(4)), 149.41 (C(6)), 145.81 (C-arom), 141.25 (C(8)), 137.00, 136.85 (C-arom), 135.60, 135.57 (CH-arom), 133.80, 133.69, 133.43 (C-arom), 132.70, 130.28, 130.25, 129.85, 129.81, 128.84, 128.18, 127.89, 127.71, 127.65, 126.78 (CH-arom), $123.52(\mathrm{C}(5)), 113.22,113.19$ (CH-arom), $87.09(\mathrm{C}(\mathrm{Ph}) 3), 86.41$

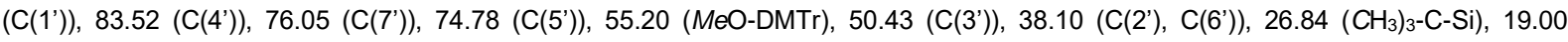
$\left(\mathrm{CH}_{3}\right)_{3}-\mathrm{C}$-Si); ESI+-HRMS $\mathrm{m} / z$ calcd for $\mathrm{C}_{56} \mathrm{H}_{56} \mathrm{O}_{6} \mathrm{~N}_{5} \mathrm{Si}\left([\mathrm{M}+\mathrm{H}]^{+}\right)$922.3994, found 922.3953.

(3'S, 5'R,7'R)-N6-Benzoyl-9-\{2', 3'-Dideoxy-3',5'-ethano-7'-hydroxy-5'-O-[(4,4'-dimethoxytriphenyl)methyl]- $\beta$-D-ribofuranosyl\}

adenine (18): To a solution of nucleoside 17 (376 mg, $0.408 \mathrm{mmol})$ in dry THF (9 mL) was added TBAF (1M in THF, $1.22 \mathrm{~mL}, 1.22 \mathrm{mmol})$ at rt. The solution was stirred for 2 days, then was diluted with satd $\mathrm{NaHCO}_{3}(25 \mathrm{~mL})$ and extracted with $\mathrm{DCM}(4 \mathrm{X} 25 \mathrm{~mL})$. The combined organic phases were dried over $\mathrm{MgSO}_{4}$, filtered and evaporated. The crude product was purified by $\mathrm{CC}(4 \% \mathrm{MeOH}$ in DCM, $+0.5 \%$ $\left.\mathrm{Et}_{3} \mathrm{~N}\right)$ to yield $18(242 \mathrm{mg}, 87 \%)$ as a white foam. Data for 18: $R_{\mathrm{f}}=0.33\left(5 \% \mathrm{MeOH}\right.$ in DCM); ${ }^{1} \mathrm{H} \mathrm{NMR}\left(300 \mathrm{MHz}, \mathrm{CD}_{3} \mathrm{CN}\right) \delta 9.35(b r, 1 \mathrm{H}$, $\mathrm{NH}$ ), 8.67 (s, 1H, C(2)), 8.46 (s, 1H, C(8)), 8.01 (d, J = 7.4 Hz, 2H, H-arom), 7.54 (m, 5H, H-arom), 7.35 (m, 4H, H-arom), 7.30 - 7.17 (m, $3 \mathrm{H}, \mathrm{H}$-arom), 6.84 (d, $J=8.9 \mathrm{~Hz}, 4 \mathrm{H}, \mathrm{H}$-arom), 6.09 (dd, $\left.J=7.8,6.2 \mathrm{~Hz}, 1 \mathrm{H}, \mathrm{H}-\mathrm{C}\left(1^{\prime}\right)\right), 4.12\left(\mathrm{dt}, J=11.2,5.8 \mathrm{~Hz}, 1 \mathrm{H}, \mathrm{C}\left(5^{\prime}\right)\right), 3.87-3.79$ $\left(\mathrm{m}, 2 \mathrm{H}, \mathrm{C}\left(4^{\prime}\right), \mathrm{C}\left(7^{\prime}\right)\right), 3.75(\mathrm{~s}, 6 \mathrm{H}, \mathrm{MeO}), 2.83-2.64\left(\mathrm{~m}, 2 \mathrm{H}, \mathrm{C}\left(2^{\prime}\right), \mathrm{OH}\right), 2.58-2.46\left(\mathrm{~m}, 1 \mathrm{H}, \mathrm{C}\left(3^{\prime}\right)\right), 2.21\left(\mathrm{dd}, J=13.9,7.1 \mathrm{~Hz}, 1 \mathrm{H}, \mathrm{C}\left(2^{\prime}\right)\right)$, $1.92-1.82\left(\mathrm{~m}, 1 \mathrm{H}, \mathrm{C}\left(6^{\prime}\right)\right), 1.29-1.17\left(\mathrm{~m}, 1 \mathrm{H}, \mathrm{C}\left(6^{\prime}\right)\right) ;{ }^{13} \mathrm{C} \mathrm{NMR}\left(75 \mathrm{MHz}, \mathrm{CDCl}_{3}\right) \delta 165.03(\mathrm{CONH}), 158.57(\mathrm{MeO}-\mathrm{C}$-arom), 152.40 (C(2)), 151.23 (C(4)), 149.52 (C(6)), 145.68 (C-arom), 141.49 (C(8)), 136.86, 136.84, 133.77 (C-arom), 132.77, 130.22, 128.81, 128.16, 128.02, 127.89, 126.84 (CH-arom), $123.40(\mathrm{C}(5)), 113.19$ (CH-arom), $87.06(\mathrm{C}(\mathrm{Ph}) 3), 86.74\left(\mathrm{C}\left(1^{\prime}\right)\right), 83.58\left(\mathrm{C}\left(4^{\prime}\right)\right), 74.62\left(\mathrm{C}\left(5^{\prime}\right)\right), 74.38$

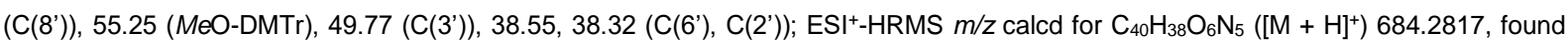
684.2830

(3'R, 5'R, 7'R)-N6-Benzoyl-9-\{7'-O-[(2-cyanoethoxy)-diisopropylaminophosphanyl]-2',3'-Dideoxy-3',5'-ethano-5'-O-[(4,4'dimethoxytriphenyl)methyl]- $\beta$-D-ribofuranosyl\} adenine (19): To a solution of the nucleoside 18 (173 mg, $0.253 \mathrm{mmol})$ and $\mathrm{N}, \mathrm{N}$ diisopropylethylamine $(0.18 \mathrm{~mL}, 1.0 \mathrm{mmol})$ in dry THF $(8 \mathrm{~mL})$ was added $\mathrm{N}, \mathrm{N}$-diisopropylchlorophosphoramidite $(0.11 \mathrm{~mL}, 0.50 \mathrm{mmol})$ at rt. The solution was stirred for 2 hours and then was diluted with satd $\mathrm{NaHCO}_{3}(40 \mathrm{~mL})$ and extracted with $\mathrm{DCM}(4 \mathrm{X} 40 \mathrm{~mL})$. The combined organic phases were dried over $\mathrm{MgSO}_{4}$, filtered and evaporated. The crude product was purified by CC (EtOAc, $\left.+0.5 \% \mathrm{Et}_{3} \mathrm{~N}\right)$ to yield 19 (177 mg, mixture of two isomers, $71 \%$ ) as a white foam. Data for 19: $R_{\mathrm{f}}=0.38,0.44(\mathrm{EtOAc}) ;{ }^{1} \mathrm{H} \mathrm{NMR}\left(400 \mathrm{MHz}, \mathrm{CDCl}_{3}\right) \delta$ 
$9.05(b r, 1 \mathrm{H}, \mathrm{NH}), 8.70,8.70(2 \mathrm{~s}, 1 \mathrm{H}, \mathrm{H}-\mathrm{C}(2)), 8.47,8.46(2 \mathrm{~s}, 1 \mathrm{H}, \mathrm{H}-\mathrm{C}(8)), 7.97$ (d, J = 7.5 Hz, 2H, H-arom), $7.57-7.50$ (m, 1H, H-arom), $7.49-7.41$ (m, 4H, H-arom), 7.39 - 7.31 (m, 4H, H-arom), $7.24-7.17$ (m, $5.4 \mathrm{~Hz}, 2 \mathrm{H}, \mathrm{H}$-arom), 7.13 (dt, J=12.5, 6.2 Hz, 1H, H-arom), $6.83-6.70$ (m, 4H, H-arom), $6.14-5.97\left(\mathrm{~m}, 1 \mathrm{H}, \mathrm{H}-\mathrm{C}\left(1^{\prime}\right)\right), 4.14$ (ddd, J =11.1, 7.8, 3.4 Hz, 1H, H-C(5')), $3.91-3.74\left(\mathrm{~m}, 2 \mathrm{H}, \mathrm{H}-\left(4^{\prime}\right), \mathrm{H}-\right.$ $\left.\mathrm{C}\left(7^{\prime}\right)\right)$, $3.71,3.70(2 \mathrm{~s}, 6 \mathrm{H}, \mathrm{MeO}), 3.65-3.50\left(\mathrm{~m}, 2 \mathrm{H}, \mathrm{OCH}_{2} \mathrm{CH}_{2} \mathrm{CN}\right.$ ), 3.37 (ddq, J = 13.9, 10.2, $\left.6.8 \mathrm{~Hz}, 2 \mathrm{H},\left(\mathrm{Me}_{2} \mathrm{CH}\right)_{2} \mathrm{~N}\right), 2.90-2.76(\mathrm{~m}$, $\left.1 \mathrm{H}, \mathrm{H}-\mathrm{C}\left(2^{\prime}\right)\right), 2.75-2.60\left(\mathrm{~m}, 1 \mathrm{H}, \mathrm{H}-\mathrm{C}\left(3^{\prime}\right)\right), 2.47,2.42\left(2 \mathrm{t}, J=6.3 \mathrm{~Hz}, 2 \mathrm{H}, \mathrm{OCH}_{2} \mathrm{CH}_{2} \mathrm{CN}\right.$ ), $2.11\left(\mathrm{dt}, J=12.7,6.1 \mathrm{~Hz}, 1 \mathrm{H}, \mathrm{H}-\mathrm{C}\left(2^{\prime}\right)\right), 1.73$ (ddt, $\left.J=13.6,10.4,5.1 \mathrm{~Hz}, 1 \mathrm{H}, \mathrm{H}-\mathrm{C}\left(6^{\prime}\right)\right), 1.39$ (ddd, $\left.J=50.2,13.4,6.2 \mathrm{~Hz}, 1 \mathrm{H}, \mathrm{H}-\mathrm{C}\left(6^{\prime}\right)\right), 1.10-0.89(\mathrm{~m}, 12 \mathrm{H},(M e 2 \mathrm{CH}) 2 \mathrm{~N}) ;{ }^{13} \mathrm{C} \mathrm{NMR}$ (101 MHz, $\mathrm{CDCl}_{3}$ ) $\delta 164.66$ (CONH), 158.57 (MeO-C-arom), 152.46 (C(2)), 151.32, 151.26 (C(4)), 149.45, 149.43 (C(6)), 145.60, 145.59 (C-arom), 141.52, 141.47 (C(8)), 136.88, 136.83, 136.81, 133.78 (C-arom), 132.75, 132.73, 130.22, 130.21, 130.19, 130.17, 128.87, 128.17, 127.87, 126.82, 126.80 (CH-arom), $123.59(\mathrm{C}(5)), 117.53,117.50\left(\mathrm{OCH}_{2} \mathrm{CH}_{2} \mathrm{CN}\right), 113.17$ ( $\mathrm{CH}$-arom), 87.10, $87.07(\mathrm{C}(\mathrm{Ph}) 3)$, 86.72, $86.68\left(C\left(1^{\prime}\right)\right), 83.36,83.25\left(C\left(4^{\prime}\right)\right), 76.55,75.81$ ( JC,P = 16.9, $\left.15.7 \mathrm{~Hz}, \mathrm{C}\left(7^{\prime}\right)\right), 74.63,74.60\left(\mathrm{C}\left(5^{\prime}\right)\right), 58.24,57.86$ ( JC,P = 19.1, 19.2 $\mathrm{Hz} \mathrm{OCH} \mathrm{CH}_{2} \mathrm{CN}$ ), 55.25, 55.21 (MeO-DMTr), 49.29, 49.08 ( JC,P = 2.6, $\left.4.7 \mathrm{~Hz}, \mathrm{C}\left(3^{\prime}\right)\right)$, 43.12, 43.00 ( JC,P = 2.4, $\left.2.3 \mathrm{~Hz}\left(\mathrm{Me}_{2} \mathrm{CH}\right)_{2} \mathrm{~N}\right)$, 38.27, $38.23\left(\mathrm{C}\left(2^{\prime}\right)\right)$, 37.41, $37.22\left(\mathrm{JC}_{\mathrm{C}, \mathrm{P}}=5.3,3.5 \mathrm{~Hz}, \mathrm{C}\left(6^{\prime}\right)\right)$ 24.56, 24.53, 24.49, 24.47, 24.43, 24.41, 24.36, $\left.24.33\left(8 \mathrm{~s}, \mathrm{Me}_{2} \mathrm{CH}\right)_{2} \mathrm{~N}\right)$,

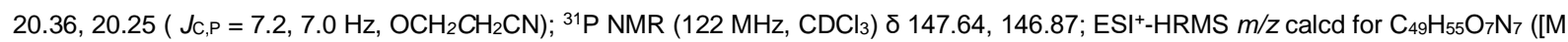
$\left.+\mathrm{H}^{+}\right) 884.3895$, found 884.3898 .

$\left(3^{\prime} R, 5^{\prime} R, 7^{\prime} R\right)-2-A m i n o-6-c h l o r o-9-\left\{5^{\prime}-O\right.$-acetyl-7'-[(tert-butyldiphenylsilyl)oxy]-2', 3'-Dideoxy-3', 5'-ethano- $\alpha, \beta$-D-ribofuranosyl\} purine (20): To a suspension of sugar 7 (1.75 g, $3.85 \mathrm{mmol})$ and 2-amino-6-chloropurine $(1.05 \mathrm{~g}, 6.17 \mathrm{mmol})$ in dry MeCN $(20 \mathrm{~mL})$ was added BSA $(3.80 \mathrm{~mL}, 15.4 \mathrm{mmol})$ at rt. The suspension was heated to $55^{\circ} \mathrm{C}$ and stirred for $30 \mathrm{~min}$. Then TMSOTf $(1.05 \mathrm{~mL}, 5.78 \mathrm{mmol})$ was added dropwise and the solution was further stirred for $50 \mathrm{~min}$ at $55^{\circ} \mathrm{C}$. The solution was cooled down to rt, quenched with addition of satd $\mathrm{NaHCO}_{3}(10 \mathrm{~mL})$, diluted with EtOAc $(50 \mathrm{~mL})$ and filtered through a short pad of $\mathrm{SiO}_{2}$. The $\mathrm{SiO}_{2}$ was washed with additional EtOAc. The mixture was then washed with satd $\mathrm{NaHCO}_{3}(2 \times 80 \mathrm{~mL})$, aqueous phases were combined and extracted with $\mathrm{EtOAc}(3 \times 50 \mathrm{~mL})$. The combined organic phases were dried over $\mathrm{MgSO}_{4}$, filtered and evaporated. The crude product was purified by $\mathrm{CC}(2.5 \% \mathrm{MeOH}$ in $\mathrm{DCM})$ to yield a mixture of $20(1.77 \mathrm{~g}, 77 \%)$ in an anomeric ratio $\alpha / \beta \approx 7: 3$ as a white foam. Data for $20: R \mathrm{f}=0.54$ (EtOAc/hexane 5:1); ${ }^{1} \mathrm{H}$ NMR (300 MHz, CDCl $)$ ) 7.86 (s, 0.3H, H-C(8)), 7.69 (s, 0.7H, H-C(8)), $7.68-7.60$ (m, 4H, H-arom), 7.47 - 7.34 (m, 6H, H-arom), 6.04 (dd, J $\left.=6.9,3.0 \mathrm{~Hz}, 0.7 \mathrm{H}, \mathrm{H}-\mathrm{C}\left(1^{\prime}\right)\right), 5.87\left(\mathrm{dd}, J=8.0,6.2 \mathrm{~Hz}, 0.3 \mathrm{H}, \mathrm{H}-\mathrm{C}\left(1^{\prime}\right)\right), 5.37\left(\mathrm{dt}, J=14.2,4.6 \mathrm{~Hz}, 1 \mathrm{H}, \mathrm{H}-\mathrm{C}\left(5^{\prime}\right)\right), 5.16\left(b r, 2 \mathrm{H}, \mathrm{NH} \mathrm{H}_{2}\right), 4.91$ $\left(\mathrm{dd}, J=6.5,5.1 \mathrm{~Hz}, 0.7 \mathrm{H}, \mathrm{H}-\mathrm{C}\left(4^{\prime}\right)\right), 4.79\left(\mathrm{dd}, J=6.9,5.2 \mathrm{~Hz}, 0.3 \mathrm{H}, \mathrm{H}-\mathrm{C}\left(4^{\prime}\right)\right), 4.13\left(b r, 0.3 \mathrm{H}, \mathrm{H}-\mathrm{C}\left(7^{\prime}\right)\right), 4.06\left(\mathrm{~d}, J=4.0 \mathrm{~Hz}, 0.7 \mathrm{H}, \mathrm{H}-\mathrm{C}\left(7^{\prime}\right)\right)$, $2.95\left(\mathrm{dd}, J=16.3,6.6 \mathrm{~Hz}, 0.7 \mathrm{H}, \mathrm{H}-\mathrm{C}\left(3^{\prime}\right)\right), 2.81\left(\mathrm{dd}, J=17.0,7.4 \mathrm{~Hz}, 0.3 \mathrm{H}, \mathrm{H}-\mathrm{C}\left(3^{\prime}\right)\right), 2.49-2.30\left(\mathrm{~m}, 1 \mathrm{H}, \mathrm{H}-\mathrm{C}\left(2^{\prime}\right)\right), 2.14(\mathrm{dd}, J=13.1,6.7$ $\left.\mathrm{Hz}, 1 \mathrm{H}, \mathrm{H}-\mathrm{C}\left(6^{\prime}\right)\right), 2.08$ (s, 2.1H, $\mathrm{MeCO}_{2}$ ), $2.02\left(\mathrm{~s}, 0.9 \mathrm{H}, \mathrm{MeCO}_{2}\right), 2.02-1.91$ (m, $\left.1 \mathrm{H}, \mathrm{H}-\mathrm{C}\left(6^{\prime}\right)\right), 1.80$ (td, $\left.J=13.4,6.8 \mathrm{~Hz}, 1 \mathrm{H}, \mathrm{H}-\mathrm{C}\left(2^{\prime}\right)\right)$, 1.07, 1.06 (2s, 9H, $\left.\left(\mathrm{CH}_{3}\right)_{3}-\mathrm{C}-\mathrm{Si}\right) ;{ }^{13} \mathrm{C} \mathrm{NMR}\left(75 \mathrm{MHz}, \mathrm{CDCl}_{3}\right) \delta$ 170.55, $170.44\left(\mathrm{MeCO}_{2}\right), 158.98,158.91$ (C(2)), 153.18, 152.95 (C(4)), 151.40, 151.34 (C(6)), 140.38, 140.14 (C(8)), 135.73, 135.70 (CH-arom), 133.78, 133.62, 133.24, 133.17 (C-arom), 130.03, 130.00, 127.88, 127.86 (CH-arom), 125.65, $125.57(\mathrm{C}(5)), 86.59,85.74\left(\mathrm{C}\left(1^{\prime}\right)\right), 82.93,80.99\left(\mathrm{C}\left(4^{\prime}\right)\right), 76.57,76.14\left(\mathrm{C}\left(7^{\prime}\right)\right), 74.34,74.32\left(\mathrm{C}\left(5^{\prime}\right)\right)$,

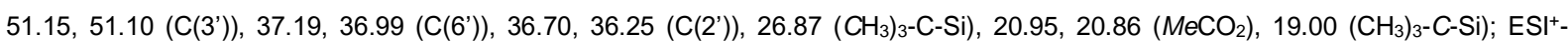
HRMS $m / z$ calcd for $\mathrm{C}_{30} \mathrm{H}_{35} \mathrm{O}_{4} \mathrm{~N}_{5} \mathrm{ClSi}\left(\left[\mathrm{M}+\mathrm{H}^{+}\right)\right.$592.2141, found 592.2158 .

$\left(3^{\prime} R, 5^{\prime} R, 7^{\prime} R\right)$ - 2-Amino-6-chloro-9-\{7'-[(tert-butyldiphenylsilyl)oxy]-2',3'-Dideoxy-3',5'-ethano- $\beta$-D-ribofuranosyl\} purine (21): The nucleoside $20(1.78 \mathrm{~g}, 3.01 \mathrm{mmol})$ was dissolved in $0.5 \mathrm{M} \mathrm{NaOH}$ in $\mathrm{THF} /$ methanol/ $\mathrm{H}_{2} \mathrm{O}(5: 4: 1,15 \mathrm{~mL})$ at $0^{\circ} \mathrm{C}$. The reaction was stirred for $20 \mathrm{~min}$ at $0^{\circ} \mathrm{C}$ and quenched by addition of $\mathrm{NH}_{4} \mathrm{Cl}(484 \mathrm{mg})$. The suspension was then diluted with satd $\mathrm{NaHCO}_{3}(100 \mathrm{~mL})$ and extracted with DCM (4 X75 mL). The combined organic phases were dried over $\mathrm{MgSO}_{4}$, filtered and evaporated. The crude product was purified by CC (3\% MeOH in DCM) to yield 21 (428 mg, 25\%) and its corresponding a anomer (992 mg, 60\%) as white foams; Data for 21: $R_{\mathrm{f}}=0.43\left(5 \% \mathrm{MeOH}\right.$ in DCM); ${ }^{1} \mathrm{H}$ NMR $\left(300 \mathrm{MHz}, \mathrm{CDCl}_{3}\right) \delta 7.71(\mathrm{~s}, 1 \mathrm{H}, \mathrm{H}-\mathrm{C}(8)), 7.68-7.60(\mathrm{~m}, 4 \mathrm{H}, \mathrm{H}$-arom), $7.44-7.33(\mathrm{~m}, 6 \mathrm{H}$, H-arom), 5.85 (dd, J = 9.3, $\left.5.8 \mathrm{~Hz}, 1 \mathrm{H}, \mathrm{H}-\mathrm{C}\left(1^{\prime}\right)\right), 5.33\left(b r, 2 \mathrm{H}, \mathrm{NH}_{2}\right.$ ), 4.62 (dd, J = 8.4, 4.9 Hz, 1H, H-C(4')), 4.44 (dd, J = 10.7, $5.3 \mathrm{~Hz}$,

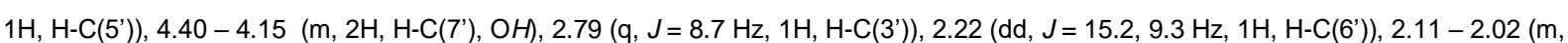


1H, H-C(6')), $2.02-1.85$ (m, 2H, H-C(2')), 1.06 (s, 9H, ( $\left.\left.\mathrm{CH}_{3}\right)_{3}-\mathrm{C}-\mathrm{Si}\right) ;{ }^{13} \mathrm{C}$ NMR $\left(75 \mathrm{MHz}, \mathrm{CDCl}_{3}\right) \delta 158.73(\mathrm{C}(2)), 152.78(\mathrm{C}(4)), 151.94$ (C(6)), 140.70 (C(8)), 135.70 (CH-arom), 133.91, 133.48 (C-arom), 129.90, 127.78 (CH-arom), 125.97 (C(5)), 87.96 (C(1')), 82.88 $\left(\mathrm{C}\left(5^{\prime}\right)\right), 76.85$ (C(7')), $\left.\left.72.36\left(\mathrm{C}\left(5^{\prime}\right)\right), 50.41\left(\mathrm{C}\left(3^{\prime}\right)\right), 41.96\left(\mathrm{C}\left(6^{\prime}\right)\right), 35.73\left(\mathrm{C}\left(2^{\prime}\right)\right), 26.90\left(\mathrm{CH}_{3}\right)_{3}-\mathrm{C}-\mathrm{Si}\right), 19.02\left(\mathrm{CH}_{3}\right)_{3}-\mathrm{C}-\mathrm{Si}\right)$; ESI+-HRMS m/z calcd for $\mathrm{C}_{28} \mathrm{H}_{33} \mathrm{O}_{3} \mathrm{~N}_{5} \mathrm{ClSi}\left([\mathrm{M}+\mathrm{H}]^{+}\right)$550.2036, found 550.2015 .

$\left(3^{\prime} R, 5^{\prime} R, 7^{\prime} R\right)-N 2-(N, N-D i m e t h y l f o r m a m i d i n o)-9-\left\{7^{\prime}-[(\right.$ tert-butyldiphenylsilyl)oxy]-2', 3'-Dideoxy-3', 5'-ethano- $\beta$-D-ribofuranosyl\} guanine (22): To a solution of 21 (380 mg, $0.645 \mathrm{mmol}$ ) and 3-hydroxypropionitrile (0.22 mL, $3.23 \mathrm{mmol})$ in dry DCM (15 mL) was added 1,5,7triazabicyclo[4.4.0]dec-5-ene $(400 \mathrm{mg}, 2.87 \mathrm{mmol})$ at $0^{\circ} \mathrm{C}$. The solution was stirred for 3 hours at $0^{\circ} \mathrm{C}$ and then for 2 days at rt. Reaction was stopped by addition of silica. After evaporation of solvent, the $\mathrm{SiO}_{2}$ powder was filtered, washed with $\mathrm{MeOH}$ and solvent evaporated to yield a brown foam. The crude product was dissolved in dry DMF $(5 \mathrm{~mL})$ and N,N-dimethylformamide dimethyl acetal $(0.43 \mathrm{~mL}, 3.2 \mathrm{mmol})$ was added. The solution was stirred for 2 hours at $55^{\circ} \mathrm{C}$ and then the solvents were removed under reduced pressure. The crude product was purified by CC $\left(6 \% \mathrm{MeOH}\right.$ in DCM) to yield $23(274 \mathrm{mg}, 73 \%)$ as yellowish foams. Data for 22: $R_{\mathrm{f}}=$ $0.45\left(12 \% \mathrm{MeOH}\right.$ in DCM); ${ }^{1} \mathrm{H}$ NMR $\left(300 \mathrm{MHz}, \mathrm{CDCl}_{3}\right) \delta 9.52(\mathrm{~s}, 1 \mathrm{H}, \mathrm{NH}), 8.46\left(\mathrm{~s}, 1 \mathrm{H}, \mathrm{NC} / \mathrm{N}\left(\mathrm{CH}_{3}\right)_{2}\right), 7.63(\mathrm{dd}, J=7.7,1.5 \mathrm{~Hz}, 4 \mathrm{H}, \mathrm{H}-$ arom), 7.50 (s, 1H, H-C(8)), $7.44-7.30$ (m, 6H, H-arom), 5.83 (dd, J = 9.3, 6.0 Hz, 1H, H-C(1')), 4.61 (dd, J = 8.7, 5.0 Hz, 1H, H-C(4')), $4.43-4.32\left(\mathrm{~m}, 1 \mathrm{H}, \mathrm{H}-\mathrm{C}\left(5^{\prime}\right)\right), 4.29\left(\mathrm{dd}, J=7.0,4.8 \mathrm{~Hz}, 1 \mathrm{H}, \mathrm{H}-\mathrm{C}\left(7^{\prime}\right)\right), 3.95(\mathrm{~d}, J=5.1 \mathrm{~Hz}, 1 \mathrm{H}, \mathrm{OH}), 2.98\left(\mathrm{~s}, 6 \mathrm{H}, \mathrm{NCHN}\left(\mathrm{CH}_{3}\right)_{2}\right), 2.79(\mathrm{dd}, J$ $\left.=18.0,7.0 \mathrm{~Hz}, 1 \mathrm{H}, \mathrm{H}-\mathrm{C}\left(3^{\prime}\right)\right), 2.20\left(\mathrm{dt}, J=12.8,5.4 \mathrm{~Hz}, 1 \mathrm{H}, \mathrm{H}-\mathrm{C}\left(6^{\prime}\right)\right), 2.09-1.88$ (m, 3H, H-C(6'), H-C(2')), $\left.1.05\left(\mathrm{~s}, 9 \mathrm{H},\left(\mathrm{CH}_{3}\right) 3^{-} \mathrm{C}-\mathrm{Si}\right)\right) ;{ }^{13} \mathrm{C}$ NMR (75 MHz, $\left.\mathrm{CDCl}_{3}\right) \delta 158.73(\mathrm{C}(2)), 157.79$ (C(6)), $156.91\left(\mathrm{NCHN}\left(\mathrm{CH}_{3}\right)_{2}\right), 149.84(\mathrm{C}(4)), 137.00(\mathrm{C}(8)), 135.70,135.67(\mathrm{CH}$-arom), 133.78, 133.60 (C-arom), 129.93, 129.86, 127.78, 127.72 (CH-arom), 121.61 (C(5)), 88.04 (C(1')), 82.21 (C(4')), 77.49 (C(7')), 71.94 $\left(\mathrm{C}\left(5^{\prime}\right)\right), 50.13\left(\mathrm{C}\left(3^{\prime}\right)\right), 42.23\left(\mathrm{C}\left(6^{\prime}\right)\right), 41.20\left(\mathrm{NCHN}\left(\mathrm{CH}_{3}\right)_{2}\right), 35.50$ (C(2')), $\left.\left.\left.34.97\left(\mathrm{NCHN} \mathrm{CH}_{3}\right)_{2}\right), 26.87\left(\mathrm{CH}_{3}\right)_{3}-\mathrm{C}-\mathrm{Si}\right), 19.02\left(\mathrm{CH}_{3}\right)_{3}-\mathrm{C}-\mathrm{Si}\right)$; ESI+-HRMS $m / z$ calcd for $\mathrm{C}_{31} \mathrm{H}_{38} \mathrm{O}_{4} \mathrm{~N}_{6} \mathrm{Si}\left([\mathrm{M}+\mathrm{H}]^{+}\right)$586.2718, found 586.2703 .

$\left(3^{\prime} R, 5^{\prime} R, 7^{\prime} R\right)-N 2-(N, N-D i m e t h y l f o r m a m i d i n o)-9-\left\{7^{\prime}-[(\right.$ tert-butyldiphenylsilyl)oxy]-2', 3'-Dideoxy-3', 5'-ethano-5'-O-[(4,4'-

dimethoxytriphenyl)methyl]- $\beta$-D-ribofuranosyl\} guanine (23): To a solution of 22 (139 mg, $0.237 \mathrm{mmol})$ in dry pyridine $(2 \mathrm{~mL})$ was added DMTr-Cl $(240 \mathrm{mg}, 0.708 \mathrm{mmol})$ in six portions over 3 hours at $\mathrm{rt}$. After stirring overnight, the orange solution was diluted with satd $\mathrm{NaHCO}_{3}(20 \mathrm{~mL})$ and extracted with DCM $(3 \times 20 \mathrm{~mL})$. The combined organic phases were dried over $\mathrm{MgSO}_{4}$, filtered and evaporated. The crude product was purified by CC $\left(4 \% \mathrm{MeOH}\right.$ in DCM, $\left.+0.5 \% \mathrm{Et}_{3} \mathrm{~N}\right)$ to yield $23(148 \mathrm{mg}, 70 \%)$ as yellowish foams. Data for 23: $R_{\mathrm{f}}=$ $0.52\left(10 \% \mathrm{MeOH}\right.$ in DCM); ${ }^{1} \mathrm{H}$ NMR $\left(400 \mathrm{MHz}, \mathrm{CDCl}_{3}\right) \delta 9.49(\mathrm{~s}, 1 \mathrm{H}, \mathrm{NH}), 8.38\left(\mathrm{~s}, 1 \mathrm{H}, \mathrm{NCHN}\left(\mathrm{CH}_{3}\right)_{2}\right), 7.80(\mathrm{~s}, 1 \mathrm{H}, \mathrm{C}(8)), 7.50-7.43(\mathrm{~m}$, 2H, H-arom), 7.42 - 7.27 (m, 10H, H-arom), 7.26 - 7.15 (m, 6H, H-arom), 7.14 - 7.08 (m, 1H, H-arom), 6.77 - 6.68 (m, 4H, H-arom), $5.78\left(\mathrm{dd}, J=8.2,5.9 \mathrm{~Hz}, 1 \mathrm{H}, \mathrm{H}-\mathrm{C}\left(1^{\prime}\right)\right), 4.25\left(\mathrm{dt}, J=11.0,5.6 \mathrm{~Hz}, 1 \mathrm{H}, \mathrm{H}-\mathrm{C}\left(5^{\prime}\right)\right), 4.14-4.03\left(\mathrm{~m}, 1 \mathrm{H}, \mathrm{H}-\mathrm{C}\left(4^{\prime}\right)\right), 3.70-3.64(\mathrm{~m}, 7 \mathrm{H}, \mathrm{MeO}, \mathrm{H}-$ C(7')), 3.00 (s, 3H, $\left.\mathrm{NCHN}\left(\mathrm{CH}_{3}\right)_{2}\right), 2.97$ (s, 3H, $\left.\mathrm{NCHN}\left(\mathrm{CH}_{3}\right)_{2}\right), 2.43$ (dd, J=16.7, 7.5 Hz, 1H, H-C(3')), 2.24 (ddd, J = 13.3, 10.1, 5.8 Hz, $\left.1 \mathrm{H}, \mathrm{H}-\mathrm{C}\left(2^{\prime}\right)\right), 1.62$ (td, $\left.J=13.1,4.3 \mathrm{~Hz}, 1 \mathrm{H}, \mathrm{H}-\mathrm{C}\left(6^{\prime}\right)\right), 1.43$ (dt, $\left.J=13.5,8.0 \mathrm{~Hz}, 1 \mathrm{H}, \mathrm{H}-\mathrm{C}\left(2^{\prime}\right)\right), 0.99$ (dd, $\left.J=13.3,6.2 \mathrm{~Hz}, 1 \mathrm{H}\right), 0.86(\mathrm{~s}, 9 \mathrm{H}$, $\left(\mathrm{CH}_{3}\right)_{3}-\mathrm{C}$-Si)); ${ }^{13} \mathrm{C} \mathrm{NMR}\left(101 \mathrm{MHz}, \mathrm{CDCl}_{3}\right) \delta$ 158.51, 158.49 (MeO-C-arom), 158.04 (C(2)), 157.91 (C(6)), $\left.156.60\left(\mathrm{NCHN} \mathrm{CH}_{3}\right)_{2}\right), 149.76$ (C(4)), 145.83, 137.12, 136.94 (C-arom), 136.01 (C(8)), 135.60, 135.59 (CH-arom), 133.81, 133.47 (C-arom), 130.32, 130.26, 129.77, 128.24, 127.82, 127.65, 127.62, 126.67 (CH-arom), $120.65(\mathrm{C}(5)), 113.13,113.09$ ( $\mathrm{CH}$-arom), $86.82(\mathrm{C}(\mathrm{Ph}) 3), 85.01\left(\mathrm{C}\left(1^{\prime}\right)\right), 82.26$ $\left(\mathrm{C}\left(4^{\prime}\right)\right), 76.14\left(\mathrm{C}\left(7^{\prime}\right)\right), 74.61\left(\mathrm{C}\left(5^{\prime}\right)\right), 55.19(\mathrm{MeO}-\mathrm{DMTr}), 50.18\left(\mathrm{C}\left(3^{\prime}\right)\right), 41.29\left(\mathrm{NCHN}\left(\mathrm{CH}_{3}\right)_{2}\right), 38.01\left(\mathrm{C}\left(6^{\prime}\right)\right), 37.76\left(\mathrm{C}\left(2^{\prime}\right)\right), 35.14$ $\left.\left.\left(\mathrm{NCHN}\left(\mathrm{CH}_{3}\right)_{2}\right) 26.8187\left(\mathrm{CH}_{3}\right)_{3}-\mathrm{C}-\mathrm{Si}\right), 19.01\left(\mathrm{CH}_{3}\right)_{3}-\mathrm{C}-\mathrm{Si}\right)$; ESI+-HRMS m/z calcd for $\mathrm{C}_{52} \mathrm{H}_{57} \mathrm{O}_{6} \mathrm{~N}_{6} \mathrm{Si}\left(\left[\mathrm{M}+\mathrm{H}^{+}\right)\right.$889.4103, found 889.4128.

(3'S, 5'R, 7'R)-N2-(N,N-Dimethylformamidino)-9-\{2',3'-Dideoxy-3',5'-ethano-7'-hydroxy-5'-O-[(4,4'-dimethoxytriphenyl)methyl]- $\beta$-Dribofuranosyl\} guanine (24): To a solution of 23 (243 mg, $0.273 \mathrm{mmol})$ in dry THF (2 mL) was added TBAF (1M in THF, $1.65 \mathrm{~mL}, 1.63$ $\mathrm{mmol})$ at rt. The solution was stirred for 7 hours and then was diluted with satd $\mathrm{NaHCO}_{3}(30 \mathrm{~mL})$ and extracted with $\mathrm{DCM}(4 \mathrm{X} 30 \mathrm{~mL})$. The combined organic phases were dried over $\mathrm{MgSO}_{4}$, filtered and evaporated. The crude product was purified by $\mathrm{CC}(7 \% \mathrm{MeOH}$ in $\left.\mathrm{DCM},+0.5 \% \mathrm{Et}_{3} \mathrm{~N}\right)$ to yield $24(155 \mathrm{mg}, 87 \%)$ as a white foam still containing traces of TBAF. Data for $24: R_{\mathrm{f}}=0.44(10 \% \mathrm{MeOH}$ in 
DCM); ${ }^{1} \mathrm{H} \mathrm{NMR}\left(400 \mathrm{MHz}, \mathrm{CDCl}_{3}\right) \delta 9.55$ (s, $\left.1 \mathrm{H}, \mathrm{NH}\right), 8.45$ (s, $\left.1 \mathrm{H}, \mathrm{NCHN}\left(\mathrm{CH}_{3}\right)_{2}\right), 8.00$ (s, 1H, H-C(8)), $7.60-7.50$ (m, 2H, H-arom), 7.49 - 7.39 (m, 4H, H-arom), 7.31 - 7.23 (m, 2H, H-arom), 7.21 - 7.12 (m, 1H, H-arom), 6.81 (d, J = 8.5 Hz, 4H, H-arom), 5.93 (dd, $J=7.5$, $\left.6.1 \mathrm{~Hz}, 1 \mathrm{H}, \mathrm{H}-\mathrm{C}\left(1^{\prime}\right)\right), 4.26\left(\mathrm{dt}, J=11.1,5.8 \mathrm{~Hz}, 1 \mathrm{H}, \mathrm{H}-\mathrm{C}\left(5^{\prime}\right)\right), 4.07-3.98\left(\mathrm{~m}, 1 \mathrm{H}, \mathrm{H}-\mathrm{C}\left(4^{\prime}\right)\right), 3.91\left(\mathrm{~d}, J=4.3 \mathrm{~Hz}, 1 \mathrm{H}, \mathrm{H}-\mathrm{C}\left(7^{\prime}\right)\right), 3.77(\mathrm{~s}, 6 \mathrm{H}$, $\mathrm{MeO}$ ), 3.14 (s, 3H, $\left.\mathrm{NCHN}\left(\mathrm{CH}_{3}\right)_{2}\right), 3.04\left(\mathrm{~s}, 3 \mathrm{H}, \mathrm{NCHN}\left(\mathrm{CH}_{3}\right)_{2}\right), 2.73$ (ddd, $\left.J=13.3,10.1,6.0 \mathrm{~Hz}, 1 \mathrm{H}, \mathrm{H}-\mathrm{C}\left(2^{\prime}\right)\right), 2.63-2.48(\mathrm{~m}, 1 \mathrm{H}, \mathrm{H}-$ $\left.\mathrm{C}\left(3^{\prime}\right)\right), 2.12(\mathrm{br}, 1 \mathrm{H}, \mathrm{OH}), 1.95-1.82\left(\mathrm{~m}, 2 \mathrm{H}, \mathrm{H}-\mathrm{C}\left(6^{\prime}\right), \mathrm{H}-\mathrm{C}\left(2^{\prime}\right)\right), 1.14\left(\mathrm{dd}, J=13.4,6.1 \mathrm{~Hz}, 1 \mathrm{H}, \mathrm{H}-\mathrm{C}\left(6^{\prime}\right)\right) ;{ }^{13} \mathrm{C} \mathrm{NMR}\left(101 \mathrm{MHz}, \mathrm{CDCl}_{3}\right) \delta$ 158.52 (MeO-C-arom), 158.12 (C(2)), 157.88 (C(6)), $156.65\left(\mathrm{NCHN}\left(\mathrm{CH}_{3}\right)_{2}\right), 149.78$ (C(4)), 145.69, 137.02, 136.99 (C-arom), 136.07 (C(8)), 130.26, 128.26, 127.82, 126.74 ( $\mathrm{CH}$-arom), 120.53 (C(5)), 113.12 (CH-arom), $86.81(\mathrm{C}(\mathrm{Ph}) 3), 85.35\left(\mathrm{C}\left(1^{\prime}\right)\right), 82.64\left(\mathrm{C}\left(4^{\prime}\right)\right), 74.61$

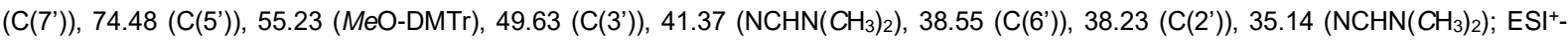
HRMS $m / z$ calcd for $\mathrm{C}_{36} \mathrm{H}_{39} \mathrm{O}_{6} \mathrm{~N}_{6}\left([\mathrm{M}+\mathrm{H}]^{+}\right) 651.2926$, found 651.2912 .

(3'R, 5'R, 7'R)-N2-(N,N-Dimethylformamidino)-9-\{7'-O-[(2-cyanoethoxy)-diisopropylaminophosphanyl]-2', 3'-Dideoxy-3',5'-ethano-5'-O[(4,4'-dimethoxytriphenyl)methyl]- $\beta$-D-ribofuranosyl\} guanine (25): To a solution of the nucleoside 24 (143 $\mathrm{mg}, 0.220 \mathrm{mmol})$ and 5-

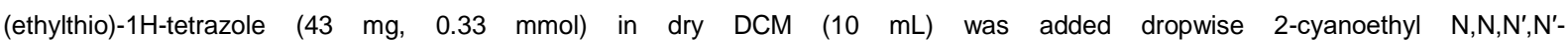
tetraisopropylphosphordiamidite $(0.12 \mathrm{~mL}, 0.38 \mathrm{mmol})$ at rt. After stirring for $50 \mathrm{~min}$, the reaction mixture was diluted with $\mathrm{satd}^{\mathrm{NaHCO}} 3$ $(20 \mathrm{~mL})$ and extracted with $\mathrm{DCM}(3 \times 20 \mathrm{~mL})$. The combined organic phases were dried over $\mathrm{MgSO}_{4}$, filtered and evaporated. The crude product was purified by CC $\left(3.5 \% \mathrm{MeOH}\right.$ in DCM, $\left.+0.5 \% \mathrm{Et}_{3} \mathrm{~N}\right)$ to yield $25(130 \mathrm{mg}$, mixture of two isomers, $69 \%)$ as a white foam. Data for 25: $R_{\mathrm{f}}=0.60\left(10 \% \mathrm{MeOH}\right.$ in DCM); ${ }^{1} \mathrm{H} \mathrm{NMR}\left(300 \mathrm{MHz}, \mathrm{CDCl}_{3}\right) \delta 9.54,9.47(2 \mathrm{~s}, 1 \mathrm{H}, \mathrm{NH}), 8.54,8.52\left(2 \mathrm{~s}, 1 \mathrm{H}, \mathrm{NCHN}\left(\mathrm{CH}_{3}\right)_{2}\right), 8.02$. 8.00 (2s, $1 \mathrm{H}, \mathrm{H}-\mathrm{C}(8)), 7.58-7.49$ (m, 2H, H-arom), $7.46-7.36$ (m, 4H, H-arom), 7.25 (dd, J=11.0, $3.5 \mathrm{~Hz}, 2 \mathrm{H}, \mathrm{H}$-arom), $7.21-7.13$ (m, 1H, H-arom), 6.80 (dd, J = 8.8, 2.2 Hz, 4H, H-arom), 6.00 - 5.82 (m, 1H, H-C(1')), 4.16 (dd, J = 10.7, 5.4 Hz, 1H, H-C(5')), $4.00-$ $3.82\left(\mathrm{~m}, 2 \mathrm{H}, \mathrm{H}-\mathrm{C}\left(4^{\prime}\right), \mathrm{H}-\mathrm{C}\left(7^{\prime}\right)\right), 3.77,3.77(2 \mathrm{~s}, 6 \mathrm{H}, \mathrm{MeO}), 3.62\left(\mathrm{dt}, J=12.2,6.1 \mathrm{~Hz}, 2 \mathrm{H}, \mathrm{OCH}_{2} \mathrm{CH}_{2} \mathrm{CN}\right), 3.51-3.33\left(\mathrm{~m}, 2 \mathrm{H},\left(\mathrm{Me}_{2} \mathrm{CH}\right)_{2} \mathrm{~N}\right)$, 3.15, $3.14\left(2 \mathrm{~s}, 3 \mathrm{H}, \mathrm{NCHN}\left(\mathrm{CH}_{3}\right)_{2}\right), 3.07$ (s, 3H, NCHN(CH3)2), $2.85-2.61\left(\mathrm{~m}, 2 \mathrm{H}, \mathrm{C}\left(2^{\prime}\right), \mathrm{C}\left(3^{\prime}\right)\right), 2.59-2.44\left(\mathrm{~m}, 2 \mathrm{H}, \mathrm{OCH}_{2} \mathrm{CH}_{2} \mathrm{CN}\right), 2.00-$ $1.79\left(\mathrm{~m}, 2 \mathrm{H}, \mathrm{H}-\left(\mathrm{C} 2^{\prime}\right), \mathrm{H}-\mathrm{C}\left(6^{\prime}\right)\right), 1.53-1.26\left(\mathrm{~m}, 1 \mathrm{H}, \mathrm{H}-\mathrm{C}\left(6^{\prime}\right)\right), 1.10,1.01\left(2 \mathrm{t}, \mathrm{J}=6.4 \mathrm{~Hz}, 12 \mathrm{H},\left(\mathrm{Me}_{2} \mathrm{CH}\right)_{2} \mathrm{~N}\right) ;{ }^{13} \mathrm{C} \mathrm{NMR}\left(101 \mathrm{MHz}, \mathrm{CDCl}_{3}\right) \delta$ 158.50 (MeO-C-arom), 158.04, 158.00 (C(2)), 157.93 (C(6)), 156.61, $156.60\left(\mathrm{NCHN}\left(\mathrm{CH}_{3}\right)_{2}\right), 149.73,149.72(\mathrm{C}(4)), 145.62,145.62$, 136.97, 136.94 (C-arom), 136.14 (C(8)), 130.27, 130.24, 130.22, 128.26, 127.81, 126.73 (CH-arom), 120.81, 120.76 (C(5)), 117.67 , $117.56\left(\mathrm{OCH}_{2} \mathrm{CH}_{2} \mathrm{CN}\right), 113.10$ ( $\mathrm{CH}$-arom), 86.88, $86.85\left(\mathrm{C}(\mathrm{Ph})_{3}\right), 85.58,85.37\left(\mathrm{C}\left(1^{\prime}\right)\right), 82.41,82.07\left(\mathrm{C}\left(4^{\prime}\right)\right), 77.08,76.01\left(J_{C, P}=37.0\right.$, $\left.15.1 \mathrm{~Hz}, \mathrm{C}\left(7^{\prime}\right)\right), 74.52,74.46\left(\mathrm{C}\left(5^{\prime}\right)\right), 58.19,57.74\left(J_{\mathrm{C}, \mathrm{P}}=18.9,19.0 \mathrm{~Hz} \mathrm{OCH} \mathrm{CH}_{2} \mathrm{CN}\right), 55.25,55.21$ (MeO-DMTr), 49.10, 48.83 ( JC,P = 2.2, $\left.4.8 \mathrm{~Hz}, \mathrm{C}\left(3^{\prime}\right)\right), 43.12,43.00\left(\left(\mathrm{Me}_{2} \mathrm{CH}\right)_{2} \mathrm{~N}\right), 41.34,41.33\left(\mathrm{NCHN}\left(\mathrm{CH}_{3}\right)_{2}\right), 38.48,38.41\left(\mathrm{C}\left(2^{\prime}\right)\right), 37.23,36.92\left(\mathrm{~J}_{\mathrm{C}, \mathrm{P}}=5.7,3.3 \mathrm{~Hz} \mathrm{C}\left(6^{\prime}\right)\right)$, 35.17 (( $\left.\left.\left.\mathrm{Me}_{2} \mathrm{CH}\right)_{2} \mathrm{~N}\right), 24.56,24.53,24.48,24.47,24.43,25.36,24.35(7 \mathrm{~s}, \mathrm{Me} 2 \mathrm{CH})_{2} \mathrm{~N}\right), 20.39,20.28\left(\mathrm{JC}_{\mathrm{C}, \mathrm{P}}=7.1,6.9 \mathrm{~Hz}, \mathrm{OCH}_{2} \mathrm{CH}_{2} \mathrm{CN}\right)$; ${ }^{31} \mathrm{P}$ NMR $\left(122 \mathrm{MHz}, \mathrm{CDCl}_{3}\right) \delta 147.69,146.37$; ESI ${ }^{+}$HRMS $\mathrm{m} / \mathrm{z}$ calcd for $\mathrm{C}_{45} \mathrm{H}_{56} \mathrm{O}_{7} \mathrm{~N}_{8} \mathrm{P}\left([\mathrm{M}+\mathrm{H}]^{+}\right)$851.4004, found 851.4018.

Oligonucleotide synthesis, deprotection and purification: Oligonucleotides syntheses were performed on a Pharmaci-Gene-AssemblerPlus DNA synthesizer on a $1.3 \mu \mathrm{mol}$ scale. Natural DNA phosphoramidites ( $\mathrm{dT}, \mathrm{dC}^{4 \mathrm{bz}}$, $\mathrm{dG}^{2 \mathrm{DMF}}, \mathrm{dA}^{6 \mathrm{Bz}}$ ) and solid support (dA-Q-CPG 500, dmf-dG-Q-CPG 500, Glen Unysupport 500) were purchased from Glen Research. Natural DNA phosphoramidites were prepared as a $0.1 \mathrm{M}$ solution in MeCN and were coupled using a $1.5 \mathrm{~min}$ step. Bc-DNA phosphoramidites were prepared as a $0.1 \mathrm{M}$ solution in 1,2dichloroethane and were coupled using an extended $12 \mathrm{~min}$ step. 5-(ethylthio)-1H-tetrazole (0.25 M in MeCN) was used as coupling agent. Capping, oxidation and detritylation were performed with standard conditions. Cleavage from solid support and deprotection of oligonucleotides was achieved by treatment with concentrated ammonia at $55^{\circ} \mathrm{C}$ for $16 \mathrm{~h}$. After centrifugation, the supernatants were collected, the beads further washed with $\mathrm{H}_{2} \mathrm{O}(0.5 \mathrm{~mL}$ X2) and the resulting solutions were evaporated to dryness. Fully modified sequences were further treated with $\mathrm{NaOH}\left(0.4 \mathrm{M}\right.$ in $\mathrm{H}_{2} \mathrm{O} / \mathrm{MeOH}$ 1:1) for $1 \mathrm{~h}$ at th to achieve complete cleavage from the universal support linker and were then filtrated using spin-columns (Amicon Ultra $0.5 \mathrm{~mL}$ centrifugal filters, MWCO $3 \mathrm{kDa}$ ). Crude oligonucleotides were purified by ion-exchange HPLC (Dionex - DNAPac PA200). Buffer solutions of $25 \mathrm{mM}$ Trizma in $\mathrm{H}_{2} \mathrm{O}$, pH 8.0, was used as the mobile phase "A" and $25 \mathrm{mM}$ Trizma, $1.25 \mathrm{M} \mathrm{NaCl}$ in $\mathrm{H}_{2} \mathrm{O}$, pH 8.0, was used as the mobile phase " $\mathrm{B}$ ". The purified oligonucleotides were 
then desalted with Sep-pack C-18 cartridges. Concentrations were determined by measuring the absorbance at $260 \mathrm{~nm}$ with a Nanodrop spectrophotometer, using the extinction coefficient of the corresponding natural DNA oligonucleotides. Characterizations of oligonucleotides were performed by $\mathrm{ESI}^{-}$mass spectrometry.

Temperature of melting: UV-melting experiments were recorded on a Varian Cary Bio 100 UV/vis spectrophotometer. Experiments were performed at $2 \mu \mathrm{M}$ duplex concentration, $10 \mathrm{mM} \mathrm{NaH}_{2} \mathrm{PO}_{4}$, between $0.05 \mathrm{M}$ and $1.00 \mathrm{M} \mathrm{NaCl}$ and pH adjusted to 7.0 . Samples were protected from evaporation by a covering layer of dimethylpolysiloxane. Absorbance was monitored at $260 \mathrm{~nm}$. For every experiment, three cooling-heating cycles were performed with a temperature gradient of $0.5^{\circ} \mathrm{C} / \mathrm{min}$. The maxima of the curves first derivative were extracted with Varian WinUV software and $T_{m}$ values were reported as the average of the six ramps.

Circular dichroism spectroscopy: CD-spectra were recorded on a Jasco J-715 spectropolarimeter equipped with a Jasco PFO-350S temperature controller. Sample conditions were the same as for UV-melting experiments. Spectra were recorded between 210 and 320 $\mathrm{nm}$ at a $50 \mathrm{~nm} / \mathrm{min}$ rate and the temperature was measured directly from the sample. For each experiment, a blank containing the same salt concentrations as the sample were recorded. The reported spectra were obtained by taking a smoothed average of three scans and subtracting the corresponding blank spectrum.

Molecular modeling: Conformational analyses of the monomers were carried out with the Gaussian 09 software package. Stepwise potential energy surface scans were performed by varying the $v 1$ and $v 3$ angles. These calculations have be done with the Hartree-Fock methodology and a $6-31 \mathrm{G}^{*}$ basis set. The solvation energies were calculated using the Self Consistent Reaction Field with the default settings. The atomic charges of four nucleobase units were then calculated using the R.E.D. III.5 tools package. The duplex structures have been constructed with Discovery Studio 4.1, starting from a natural DNA duplex in B conformation. Molecular dynamics simulations were carried out with the GROMACS 5.0.6 simulation package. The Amber94 force field was manually implemented with parameters for Bc-DNA and was used for the simulations. The duplexes were solvated in a rhombic dodecahedron with at least $1.0 \mathrm{~nm}$ to the border. The systems were filled with water (TIP3P) and neutralization was performed by addition of $\mathrm{Na}^{+}$counter-ions. $1.4 \mathrm{~nm}$ cutoffs were applied for short-range electrostatic and van der Waals forces. Long-range electrostatics interactions were calculated using the Particle Mesh Ewald method. The neutral structures were then relaxed by energy minimization (steepest descent minimization). The simulations were run with a time step of 2 fs computed by leap-frog integrator, in periodic boundary conditions and by using the LINCS algorithm. Water molecules were equilibrated by a 100 ps run at $300 \mathrm{~K}$ in NVT condition, followed by a 100 ps run at $300 \mathrm{~K}$ in NPT conditions. Atomic positions of the duplexes were restrained during these water equilibration steps. Simulations were then carried out under NPT conditions at $300 \mathrm{~K}$ and without restrains. The system was equilibrated during $10 \mathrm{~ns}$ and then data were collected over a $50 \mathrm{~ns}$ run. MD trajectories were analyzed with the GROMACS package and with 3dna package. ${ }^{[31]}$

\section{Acknowledgements}

We thank the Swiss National Science Foundation (grant-no.: 200020-146646) for generous financial support of this work. We also thank the group of Chemical Crystallography of the University of Bern (PD Dr. P. Macchi) for the X-ray structure solution and the Swiss National Science Foundation (R'equip project 206021_128724) for co-funding the single crystal X-ray diffractometer at the Department of Chemistry and Biochemistry of the University of Bern. 


\section{References}

[1] J. D. Watson, F. H. Crick, Nature (London, U. K.) 1953, 737-738.

[2] a) K. Groebke, J. Hunziker, W. Fraser, L. Peng, U. Diederichsen, K. Zimmermann, A. Holzner, C. Leumann, A. Eschenmoser, Helv. Chim. Acta 1998, 81, 375-474; b) J. Hunziker, H. J. Roth, M. Böhringer, A. Giger, U. Diederichsen, M. Göbel, R. Krishnan, B. Jaun, C. Leumann, A. Eschenmoser, Helv. Chim. Acta 1993, 76, 259-352; c) M. Böhringer, H. J. Roth, J. Hunziker, M. Göbel, R. Krishnan, A. Giger, B. Schweizer, J. Schreiber, C. Leumann, A. Eschenmoser, Helv. Chim. Acta 1992, 75, 1416-1477.

[3] M. Beier, F. Reck, T. Wagner, R. Krishnamurthy, A. Eschenmoser, Science (Washington, DC, U. S.) 1999, $283,699-703$.

[4] K. Schoning, P. Scholz, S. Guntha, X. Wu, R. Krishnamurthy, A. Eschenmoser, Science (Washington, DC, U. S.) 2000, $290,1347-1351$.

[5] A. Eschenmoser, Angewandte Chemie International Edition 2011, 50, 12412-12472.

[6] aM. K. Schlegel, L. O. Essen, E. Meggers, J. Am. Chem. Soc. 2008, 130, 8158-8159; bL. Zhang, A. Peritz, E. Meggers, J. Am. Chem. Soc. 2005, 127, 4174-4175.

[7] P. E. Nielsen, Chem. Biodiversity 2007, 4, 1996-2002.

[8] P. Herdewijn, P. Marlière, Chem. Biodiversity 2009, 6, 791-808.

[9] P. Marlière, J. Patrouix, V. Döring, P. Herdewijn, S. Tricot, S. Cruveiller, M. Bouzon, R. Mutzel, Angewandte Chemie International Edition 2011, 50, 7109-7114.

[10] C. Hendrix, H. Rosemeyer, I. Verheggen, F. Seela, A. Van Aerschot, P. Herdewijn, Chem. Eur. J. 1997, 3, 110-120.

[11] K. Nauwelaerts, E. Lescrinier, G. Sclep, P. Herdewijn, Nucleic Acids Res. 2005, 33, 2452-2463.

[12] C. J. Wilds, M. J. Damha, Nucleic Acids Res. 2000, 28, 3625-3635.

[13] M. J. Damha, C. J. Wilds, A. Noronha, I. Brukner, G. Borkow, D. Arion, M. A. Parniak, J. Am. Chem. Soc. 1998, 120, $12976-12977$.

[14] a) A. A. Koshkin, P. Nielsen, M. Meldgaard, V. K. Rajwanshi, S. K. Singh, J. Wengel, J. Am. Chem. Soc. 1998, 120, 13252-13253; b) S. Obika, D. Nanbu, Y. Hari, J. I. Andoh, K. I. Morio, T. Doi, T. Imanishi, Tetrahedron Lett. 1998, 39, 5401-5404.

[15] V. B. Pinheiro, A. I. Taylor, C. Cozens, M. Abramov, M. Renders, S. Zhang, J. C. Chaput, J. Wengel, S.-Y. Peak-Chew, S. H. McLaughlin, P. Herdewijn, P. Holliger, Science 2012, 336, 341-344.

[16] A. I. Taylor, V. B. Pinheiro, M. J. Smola, A. S. Morgunov, S. Peak-Chew, C. Cozens, K. M. Weeks, P. Herdewijn, P. Holliger, Nature 2015, 518, 427-430.

[17] I. Anosova, E. A. Kowal, M. R. Dunn, J. C. Chaput, W. D. Van Horn, M. Egli, Nucleic Acids Res. 2015.

[18] M. A. Trujillo, D. Roux, J. P. Fueri, D. Samuel, H. L. Cailla, H. V. Rickenberg, Eur. J. Biochem. 1987, 169, 167-173.

[19] M. Wasner, D. Arion, G. Borkow, A. Noronha, A. H. Uddin, M. A. Parniak, M. J. Damha, Biochemistry 1998, 37, 7478-7486.

[20] a) H. Hashimoto, C. Switzer, J. Am. Chem. Soc. 1992, 114, 6255-6256; b) K. E. Jung, C. Switzer, J. Am. Chem. Soc. 1994, 116, 60596061 ; c) J. P. Dougherty, C. J. Rizzo, R. Breslow, J. Am. Chem. Soc. 1992, 114, 6254-6255.

[21] H. Robinson, K.-E. Jung, C. Switzer, A. H. J. Wang, J. Am. Chem. Soc. 1995, 117, 837-838.

[22] S. Sinha, P. H. Kim, C. Switzer, J. Am. Chem. Soc. 2004, 126, 40-41.

[23] a) M. L. Svendsen, J. Wengel, O. Dahl, F. Kirpekar, P. Roepstorff, Tetrahedron 1993, 49, 11341-11352; b) B. Haly, L. Bellon, V. Mohan, Y. Sanghvi, Nucleosides Nucleotides 1996, 15, 1383-1395.

[24] a) M. Bolli, H. U. Trafelet, C. Leumann, Nucleic Acids Res. 1996, 24, 4660-4667; b) M. Bolli, C. Litten, R. Schütz, C. Leumann, Chem. Biol. 1996, 3, 197-206; c) M. Tarköy, C. Leumann, Angew Chem Int Ed 1993, 32, 1432-1434.

[25] M. Tarköy, M. Bolli, B. Schweizer, C. Leumann, Helv. Chim. Acta 1993, 76, 481-510.

[26] K. C. Golden, B. T. Gregg, J. F. Quinn, Tetrahedron Letters 2010, 51, 4010-4013.

[27] a) M. Medvecky, A. Istrate, C. J. Leumann, J. Org. Chem. 2015, 80, 3556-3565; b) B. Dugovic, C. J. Leumann, Journal of Organic Chemistry 2014, 79, 1271-1279; c) J. Lietard, C. J. Leumann, J. Org. Chem. 2012, 77, 4566-4577.

[28] CCDC 1529960 contains the supplementary crystallographic data for this paper. These data can be obtained free of charge from The Cambridge Crystallographic Data Centre via www.ccdc.cam.ac.uk/data\%5Frequest/cif. 
[29] M. Petersheim, D. H. Turner, Biochemistry 1983, 22, 256-263.

[30] F. Y. Dupradeau, C. Cezard, R. Lelong, E. Stanislawiak, J. Pecher, J. C. Delepine, P. Cieplak, Nucleic Acids Res 2008,36, D360-367.

[31] X. J. Lu, W. K. Olson, Nature protocols 2008, 3, 1213-1227.

\section{Scheme and figure legends}

Figure 1. Top: comparison of backbone topologies of 2',5'-DNA, natural 3',5'-DNA and 7',5'-bicyclo(bc)-DNA (from right to left); bottom: chemical structures representing each type of topology.

Scheme 1. (a) LiHMDS, THF, $-78^{\circ} \mathrm{C}, 2 \mathrm{~h}, 74 \%$; (b) $\mathrm{NaBH}_{4}, \mathrm{NiCl}_{2}, \mathrm{EtOH}, 0^{\circ} \mathrm{C} \rightarrow \mathrm{rt}, 2 \mathrm{~h}, 90 \%$; (c) TBDPSCl, $\mathrm{I}_{2}, \mathrm{~N}-\mathrm{methylimidazole}, \mathrm{THF}, \mathrm{rt}, 3 \mathrm{~h}$, quant; (d) DiBAL-H, $\mathrm{CH}_{2} \mathrm{Cl}_{2},-78^{\circ} \mathrm{C}, 90 \mathrm{~min}, 89 \%$; (e) i) In(OTf) $3, \mathrm{MeCN} / \mathrm{H}_{2} \mathrm{O}, \mathrm{rt}, 48 \mathrm{~h}$, ii) $\mathrm{MeOH}, 6 \mathrm{~h}, 81 \%$; (f) Ac2O, DMAP, DCM, rt, 2h, 96\%; (g) i) TMSOTf, 2,6-lutidine, DCM, rt, 60 min, ii) TBAF, THF, $0^{\circ} \mathrm{C}, 20 \mathrm{~min}, 92 \%$; (h) DMTr-Cl, AgOTf, DCM/lutidine, rt, 4h, 93\%; (i) TBAF, THF, rt, 20h, quant.

Scheme 2. (a) i) Thymine, BSA, NIS, DCM, rt, 7h; ii) BusSnH, AIBN, toluene, $70^{\circ} \mathrm{C}, 30$ min, 73\%; (b) 2-Cyanoethyl-N,N,N',N'-tetraisopropyl phosphordiamidite, ETT, DCM, rt, 30 min, $70 \%$ for $12,75 \%$ for 14 ; (c) i) BSA, Triazole, $\left.\mathrm{POCl}_{3}, \mathrm{Et}_{3} \mathrm{~N}, \mathrm{CH}_{3} \mathrm{CN}, \mathrm{rt}, 5 \mathrm{~h}, \mathrm{ii}\right) 1,4-\mathrm{Dioxane}_{\mathrm{NHH}} \mathrm{OH}, \mathrm{rt}, 2 \mathrm{~h}$, iii) $\mathrm{Bz}_{2} \mathrm{O}, \mathrm{Et}_{3} \mathrm{~N}, \mathrm{DMF}, \mathrm{rt}, 20 \mathrm{~h}, 88 \%$.

Scheme 3. (a) $\mathrm{N}^{6}-$ Benzoyladenine, BSA, TMSOTf, $\mathrm{MeCN}, 70^{\circ} \mathrm{C}, 20 \mathrm{~min}, 64 \%$; (b) $\mathrm{NaOH}, \mathrm{THF} / \mathrm{MeOH} / \mathrm{H}_{2} \mathrm{O}, 0^{\circ} \mathrm{C}, 20 \mathrm{~min}, 69 \%$; (c) DMTr-Cl, pyridine, rt, 24h, 87\%; (d) TBAF, THF, rt, 48h, 87\%; (e) CEP-Cl, DIPEA, THF, rt, 2h, 71\%; (f) 2-amino-6-chloropurine, BSA, TMSOTf, MeCN, $55^{\circ} \mathrm{C}, 50 \mathrm{~min}, 77 \%$; (g) $\mathrm{NaOH}, \mathrm{THF} / \mathrm{MeOH} / \mathrm{H}_{2} \mathrm{O}, 0^{\circ} \mathrm{C}, 20 \mathrm{~min}, 85 \%$; (i) i) TBD, 3-hydroxypropionitrile, DCM, 48h, ii) $\mathrm{N}, \mathrm{N}$-dimethylformamide dimethylacetal, DMF, $55^{\circ} \mathrm{C}, 2 \mathrm{~h}, 73 \%$; (j) DMTr-Cl, pyridine, rt, 18h, 70\%; (k) TBAF, THF, rt, 7h, 87\%; (I) 2-Cyanoethyl N,N,N'N*tetraisopropylphosphordiamidite, ETT, DCM, rt, $50 \mathrm{~min}, 69 \%$.

Figure 2. X-ray structure of 7'-O-p-nitrobenzoyl-7',5'-bc-T. Hydrogen atoms are omitted for clarity.

Figure 3. UV-melting curves $(260 \mathrm{~nm})$ of the homo 7',5'-bc-DNA duplex in comparison with the corresponding natural DNA duplex. Total strand conc. $2 \mu \mathrm{M}$ in $10 \mathrm{mM} \mathrm{NaH}_{2} \mathrm{PO}_{4}, 150 \mathrm{mM} \mathrm{NaCl}, \mathrm{pH} 7.0$.

Figure 4. CD-spectra of the duplexes ON13.ON14, ON13.DNA and DNA.DNA. Experimental conditions: Total strand conc. $2 \mu \mathrm{M}$ in $10 \mathrm{mM}$ $\mathrm{NaH}_{2} \mathrm{PO}_{4}, 1.0 \mathrm{M} \mathrm{NaCl}, \mathrm{pH} 7.0,10^{\circ} \mathrm{C}$

Figure 5. The two minimum energy conformers of 7',5'-bc-T nucleosides a): C6'-endo and b): C6'-exo. Potential energy surfaces of c): the C6'endo conformer and d): the C6'-exo conformer.

Figure 6. Last frame of the 60 ns molecular dynamics simulation trajectory for a) 7',5'-bc-DNA homo-duplex b): 7',5'-bc-DNA/DNA c): DNA homoduplex. Section of an internal dinucleotides step for d) 7',5'-bc-DNA homo-duplex e): 7',5'-bc-DNA/DNA f): DNA homo-duplex; Population of torsion angle $y$ during the simulation for g): 7',5'bc-DNA homo-duplex h): 7',5'-bc-DNA/DNA duplex and i): DNA homo-duplex. Distribution of the pseudorotation phase angles for j): the 7',5'-bc-DNA homo-duplex and k): the DNA homo-duplex. 
Figure 7. Correlation between the simulated time spent in a C6'-exo conformation and the experimental $T_{\mathrm{m}}$ of ON2, ON5, ON6 and ON7 in duplex with complementary DNA.

\section{Tables}

Table 1. $T_{\mathrm{m}}$ and $\Delta T_{\mathrm{m}} / \mathrm{mod}$ data from UV-melting curves $(260 \mathrm{~nm})$ of ON1-ON11 in duplex with complementary DNA and RNA.

\begin{tabular}{|c|c|c|c|c|c|}
\hline Entry & Sequence ${ }^{[a],[b],[c]}$ & $\begin{array}{r}T_{\mathrm{m}}\left({ }^{\circ} \mathrm{C}\right) \\
\text { vs DNA }\end{array}$ & $\begin{array}{c}\Delta T_{\mathrm{m}} / \mathrm{mod} \\
\left({ }^{\circ} \mathrm{C}\right)\end{array}$ & $\begin{array}{r}T_{\mathrm{m}}\left({ }^{\circ} \mathrm{C}\right) \\
\text { vs RNA }\end{array}$ & $\begin{array}{c}\Delta T_{m} / \mathrm{mod} \\
\left({ }^{\circ} \mathrm{C}\right)\end{array}$ \\
\hline ON1 & d(GGATGTTCtCGA) & 50.3 & 1.2 & 47.6 & -1.8 \\
\hline ON2 & d(GGAtGTTCTCGA) & 49.0 & -0.1 & 47.0 & -2.4 \\
\hline ON3 & d(GGATGttCTCGA) & 46.9 & -1.1 & 45.4 & -2.0 \\
\hline ON4 & $\mathrm{d}(\mathrm{GGATGTT} \underline{\mathbf{c}}$ TCGA) & 52.2 & 3.1 & 50.4 & 1.0 \\
\hline ON5 & d(GGATGTTC̄T $\underline{\mathbf{c} G A)}$ & 53.4 & 4.3 & 49.5 & 0.1 \\
\hline ON6 & d(GGaTGTTCTC̄GA) & 45.6 & -3.5 & 50.0 & 0.6 \\
\hline ON7 & d(GGATgTTCTCGA) & 46.3 & -2.8 & 50.0 & 0.6 \\
\hline ON8 & d(GGATGTTcTCGA) & 50.7 & 1.6 & 50.0 & 0.6 \\
\hline ON9 & d(GGATGTTCTcGA) & 51.8 & 2.8 & 49.2 & -0.2 \\
\hline ON10 & d(GGATGTTcTcGA) & 51.9 & 1.4 & 48.5 & -0.9 \\
\hline ON11 & $\mathrm{d}(\mathrm{GCAtttttACCG})^{[\mathrm{d}]}$ & 33.2 & -2.9 & 27.9 & -3.2 \\
\hline
\end{tabular}

[a] total strand conc. $2 \mu \mathrm{M}$ in $10 \mathrm{mM} \mathrm{NaH}_{2} \mathrm{PO}_{4}, 150 \mathrm{mM} \mathrm{NaCl}$, pH 7.0.

[b] A,G,T,C denote natural 2'-deoxynucleosides; a,t,g,c corresponds to modified nucleosides, $\underline{\mathbf{c}}$ stands for the modified 5 -methyl cytosine nucleoside.

[c] Tm of unmodified duplexes, DNA/DNA: $49.1^{\circ} \mathrm{C}$, DNA/RNA: $49.4^{\circ} \mathrm{C}$

[d] $\mathrm{Tm}$ of unmodified duplexes, DNA/DNA: $47.5^{\circ} \mathrm{C}$, DNA/RNA: $44.0^{\circ} \mathrm{C}$

Table 2. $T_{\mathrm{m}}$ values from UV-melting curves $(260 \mathrm{~nm})$ of fully modified ON12ON15 in duplex with complementary antiparallel or parallel DNA or RNA.

\begin{tabular}{|c|c|c|c|c|c|}
\hline Entry & Sequence & $\begin{array}{c}T_{\mathrm{m}}\left({ }^{\circ} \mathrm{C}\right) \text { vs } \\
\text { DNA } \\
\text { antiparallel }\end{array}$ & $\begin{array}{c}T_{\mathrm{m}}\left({ }^{\circ} \mathrm{C}\right) \text { vs } \\
\text { RNA } \\
\text { antiparallel }\end{array}$ & $\begin{array}{c}T_{\mathrm{m}}\left({ }^{\circ} \mathrm{C}\right) \text { vs } \\
\text { DNA } \\
\text { parallel }\end{array}$ & $\begin{array}{c}T_{\mathrm{m}}\left({ }^{\circ} \mathrm{C}\right) \mathrm{vs} \\
\text { RNA } \\
\text { parallel }\end{array}$ \\
\hline ON12 & 5'-(ttttctcct)-7' & $<10$ & $<10$ & n.m..$^{[a]}$ & n.m. ${ }^{[a]}$ \\
\hline ON13 & $5^{\prime}$-(ggatgttct $\left.\underline{\text { cga }}\right)-7^{\prime}$ & 18.9 & 18.6 & $<10$ & $<10$ \\
\hline ON14 & $5^{\prime}$-(tcgagaacatcc) -7 & 15.7 & n.d..$^{[b]}$ & $<10$ & $<10$ \\
\hline ON15 & $5^{\prime}$-( cctacaagagct $)-7 '$ & 13.1 & 13.0 & $<10$ & n.d..$^{[b]}$ \\
\hline
\end{tabular}

Experimental conditions: total strand conc. $2 \mu \mathrm{M}$ in $10 \mathrm{mM} \mathrm{NaH}_{2} \mathrm{PO}_{4}, 1 \mathrm{M}$ $\mathrm{NaCl}, \mathrm{pH} 7.0$.

[a] n.m.: not measured

[b] n.d.: not detectable due to formation of a self-structure of the corresponding RNA strand with a $T_{\mathrm{m}}$ of $28^{\circ} \mathrm{C}$.

Table 3. Thermodynamic data of duplex formation.

\begin{tabular}{cccc}
\hline $\begin{array}{c}\text { 5'-GGA TGT TCT CGA } \\
\text { CCT ACA AGA GCT-5, }\end{array}$ & $\begin{array}{c}\Delta \mathrm{H} \\
{\left[\mathrm{kcal} \mathrm{mol}^{-1}\right]}\end{array}$ & $\begin{array}{c}\Delta \mathrm{S} \\
{\left[\mathrm{cal} \mathrm{mol}^{-1} \mathrm{~K}^{-1}\right]}\end{array}$ & $\begin{array}{c}\Delta \mathrm{G}^{25^{\circ} \mathrm{C}} \\
{\left[\mathrm{kcal} \mathrm{mol}^{-1}\right]}\end{array}$ \\
\hline DNA.DNA & -91.7 & -257.9 & -14.8 \\
ON13.ON14 & -77.0 & -208.0 & -15.0 \\
\hline
\end{tabular}

\section{Schemes and Figures:}


Scheme 1.
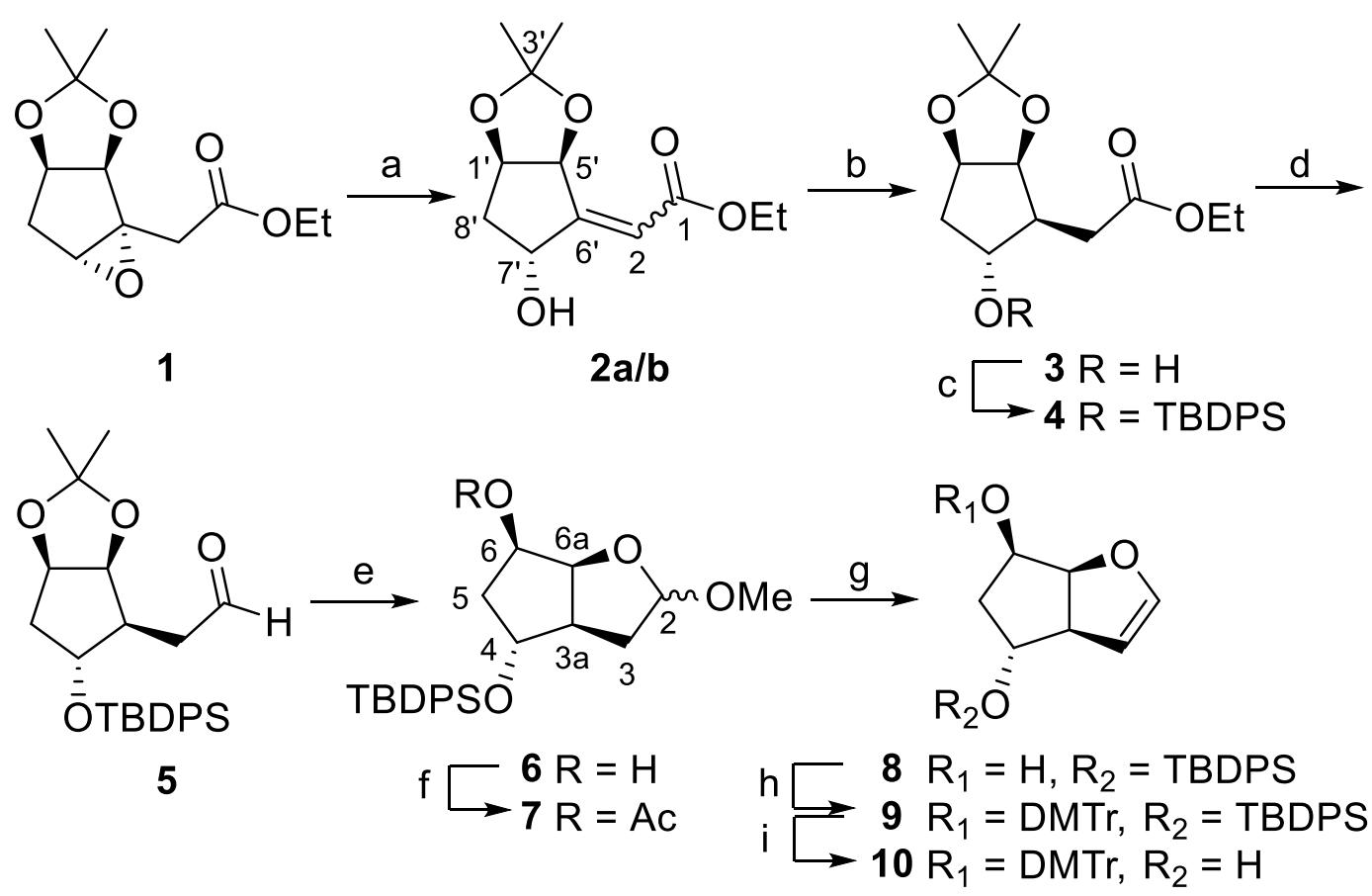

Scheme 2.

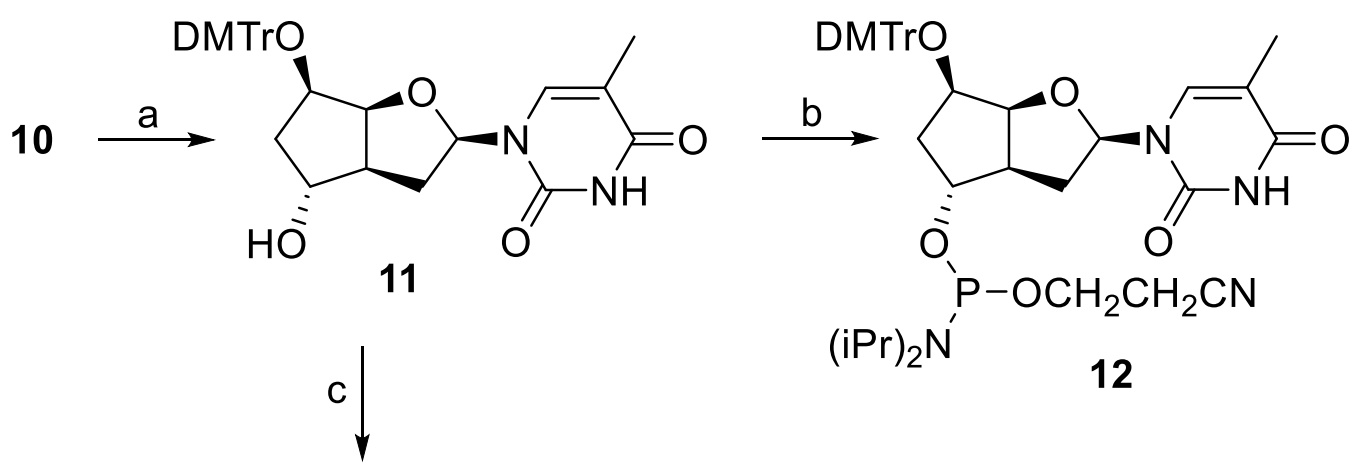

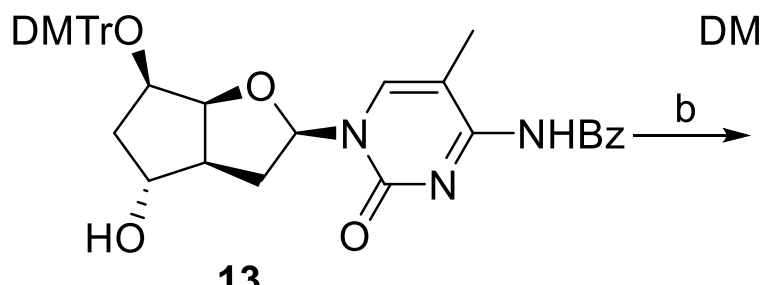

13 $(\mathrm{iPr})_{2} \mathrm{~N} \quad \mathrm{~N}^{\mathrm{P}}-\mathrm{OCH}_{2} \mathrm{CH}_{2} \mathrm{CN}$ 
Scheme 3.

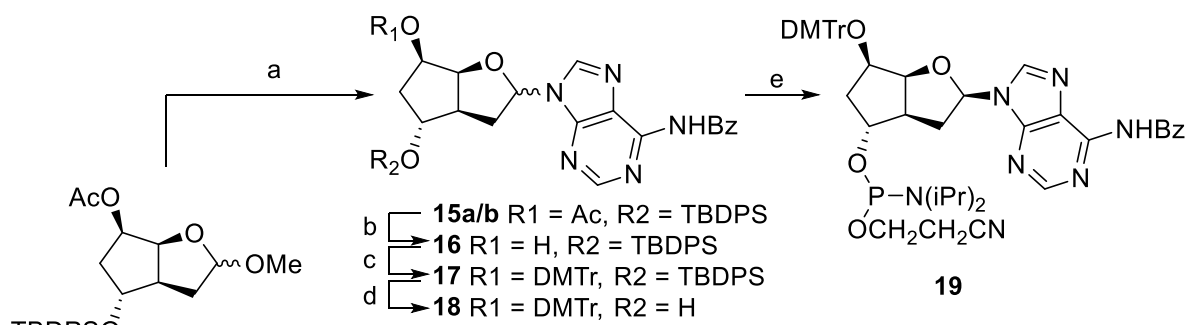

TBDPSO 7
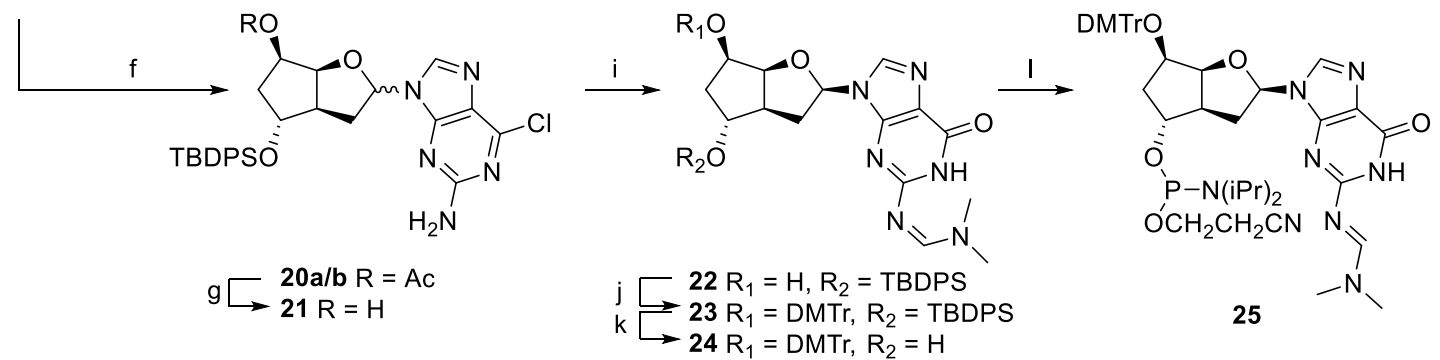

Figure 1.
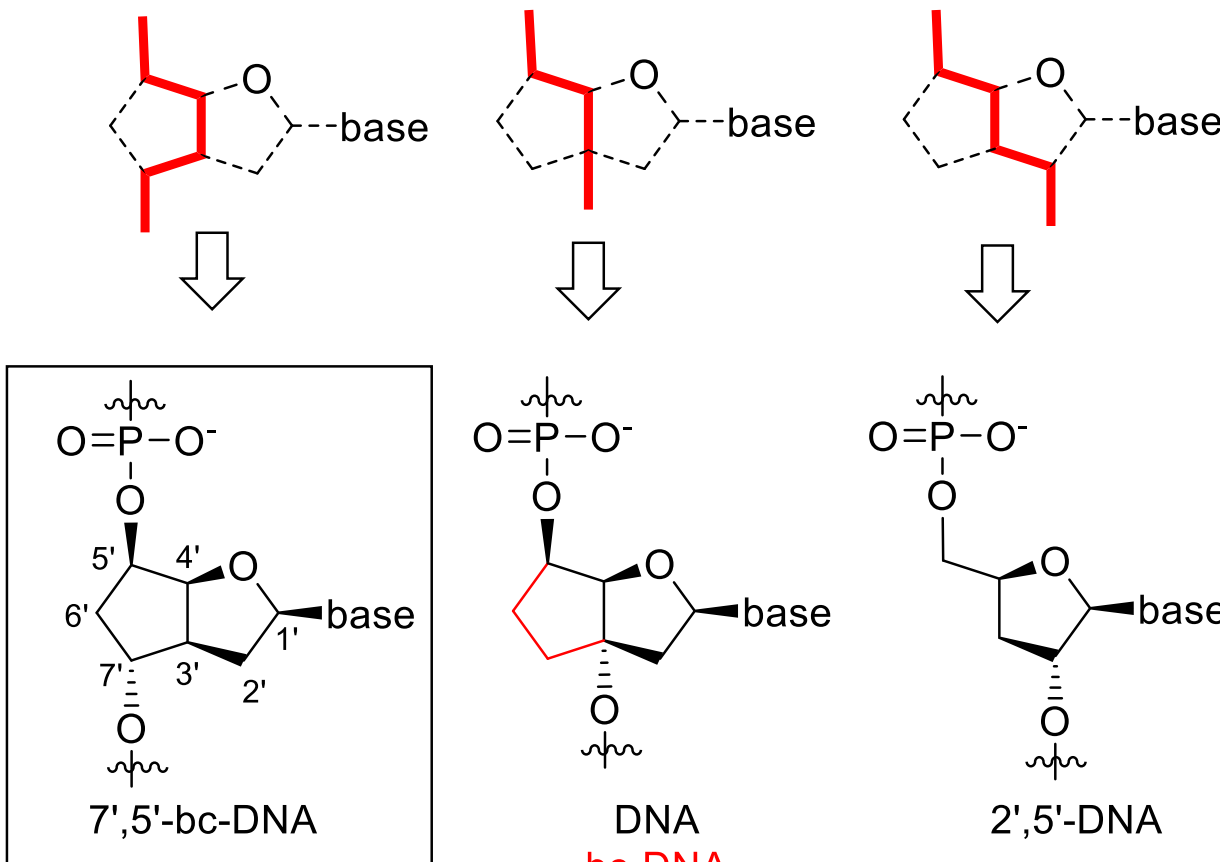

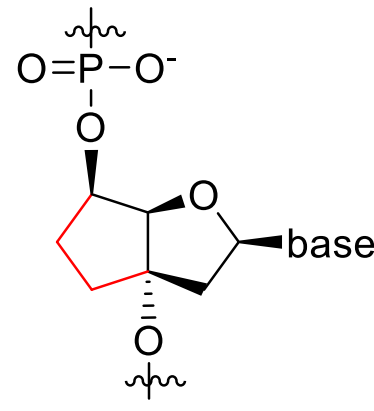

DNA bc-DNA

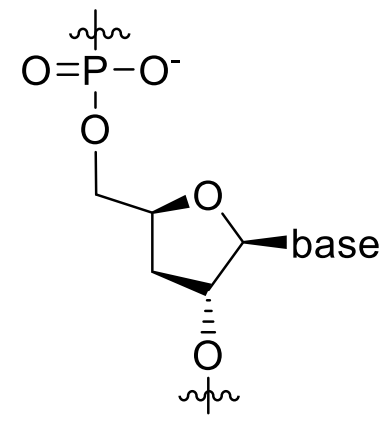

2',5'-DNA 
Figure 2.

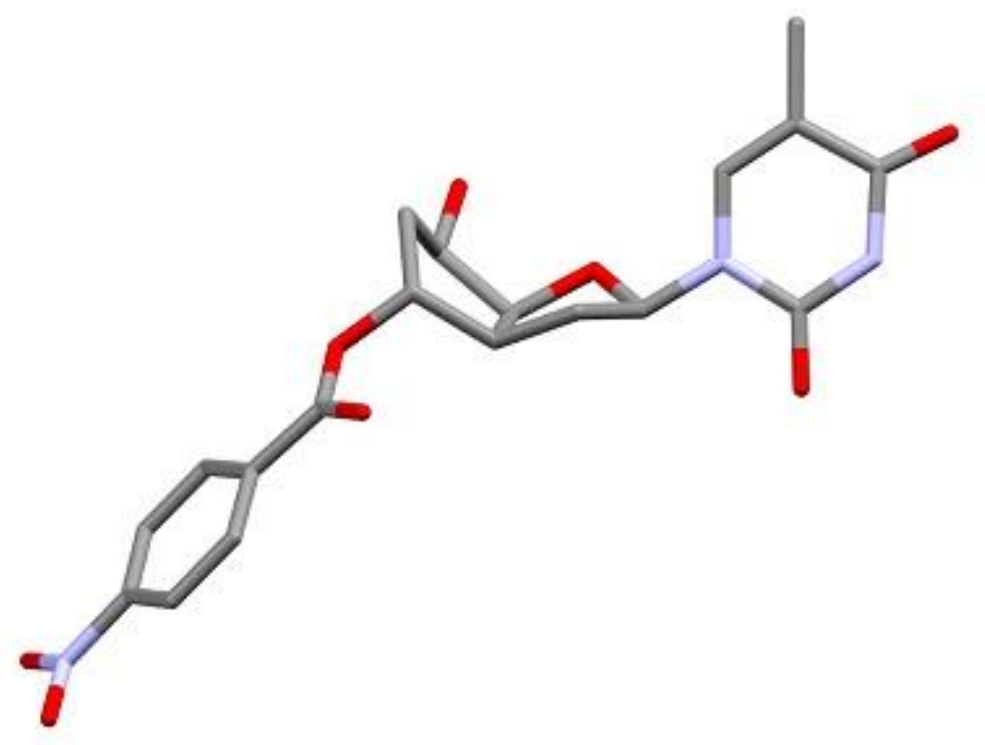

Figure 3.

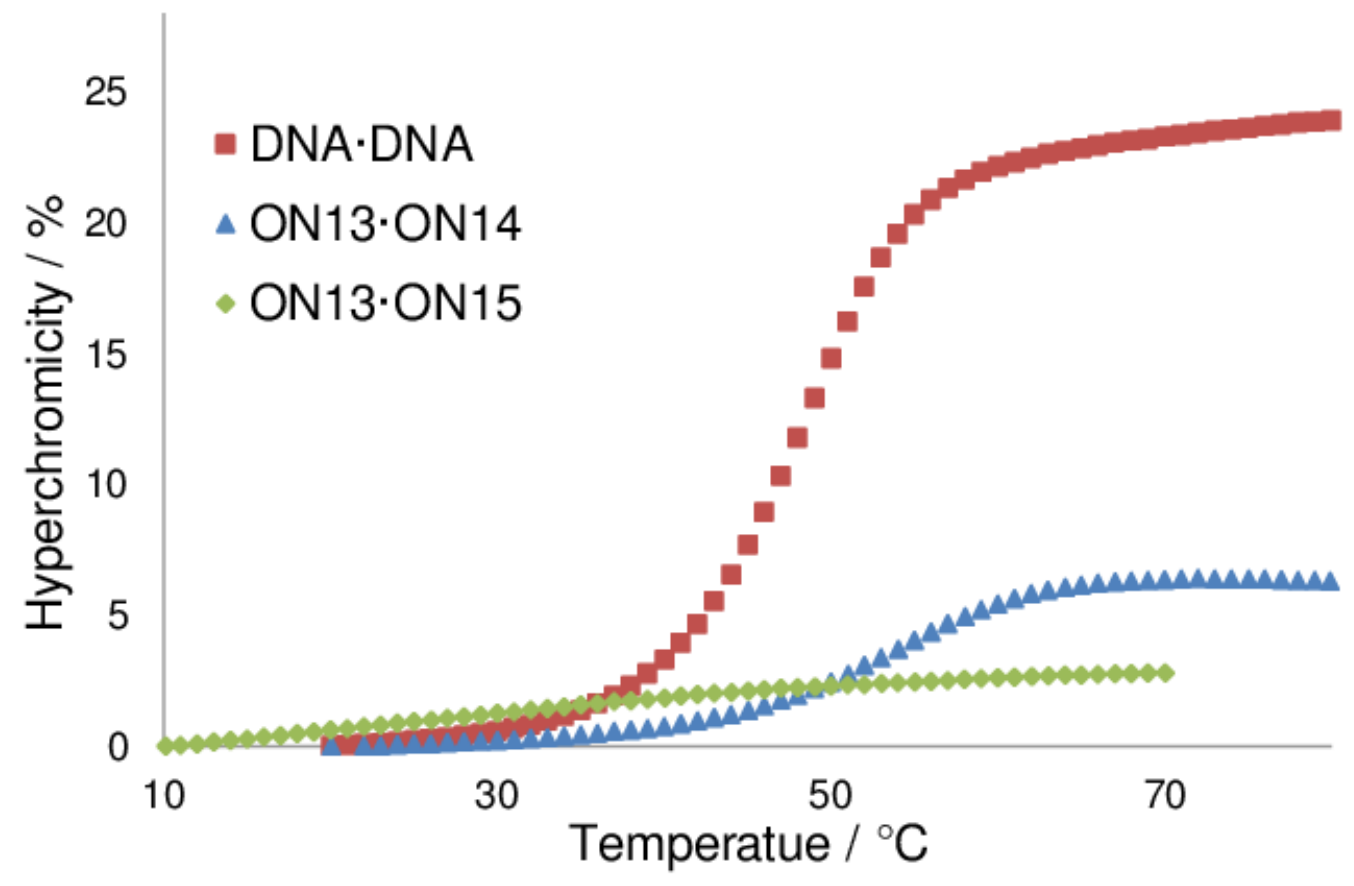


Figure 4.

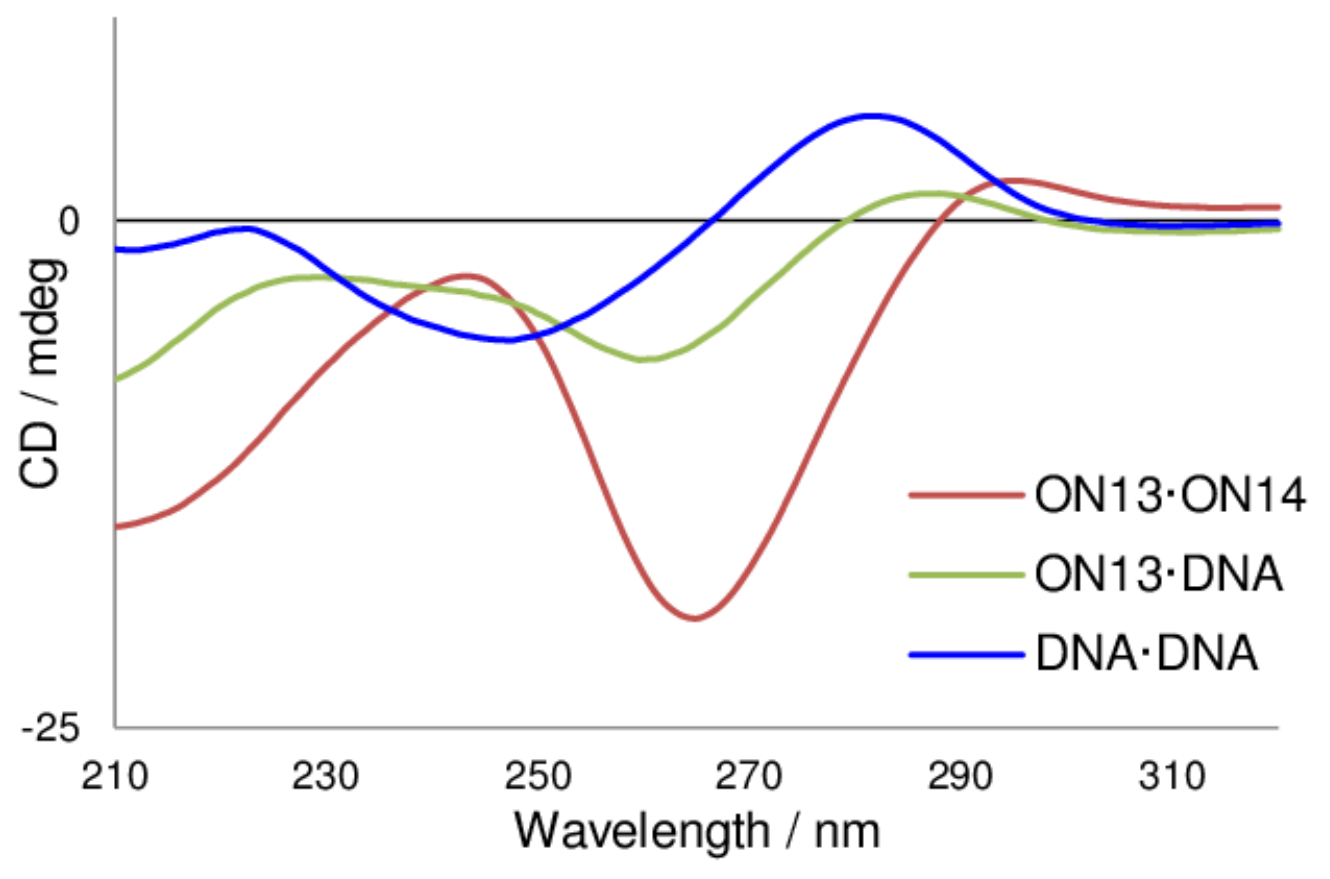

Figure 5.
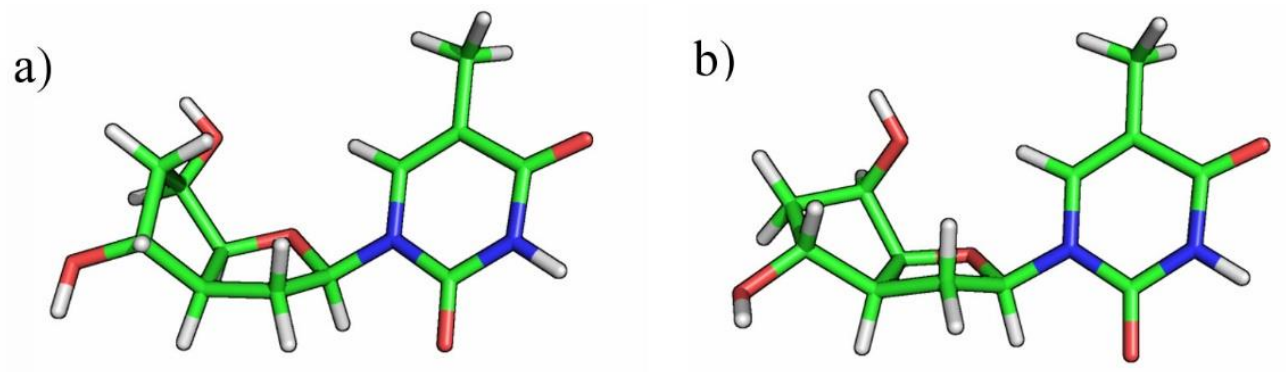

c)

d)
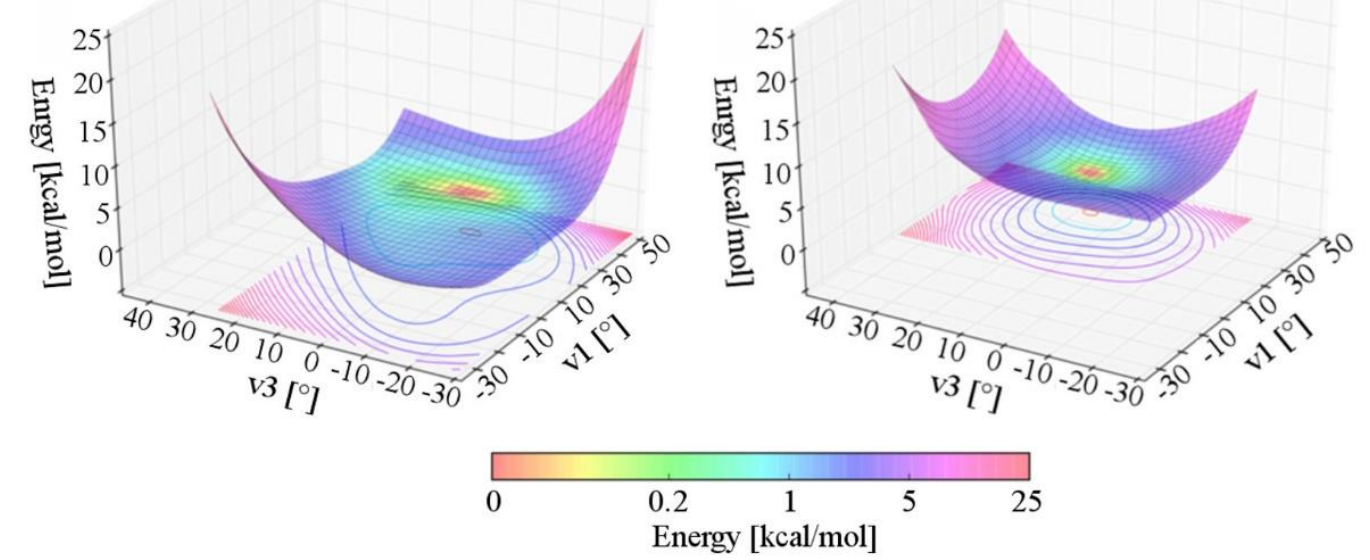
Figure 6.
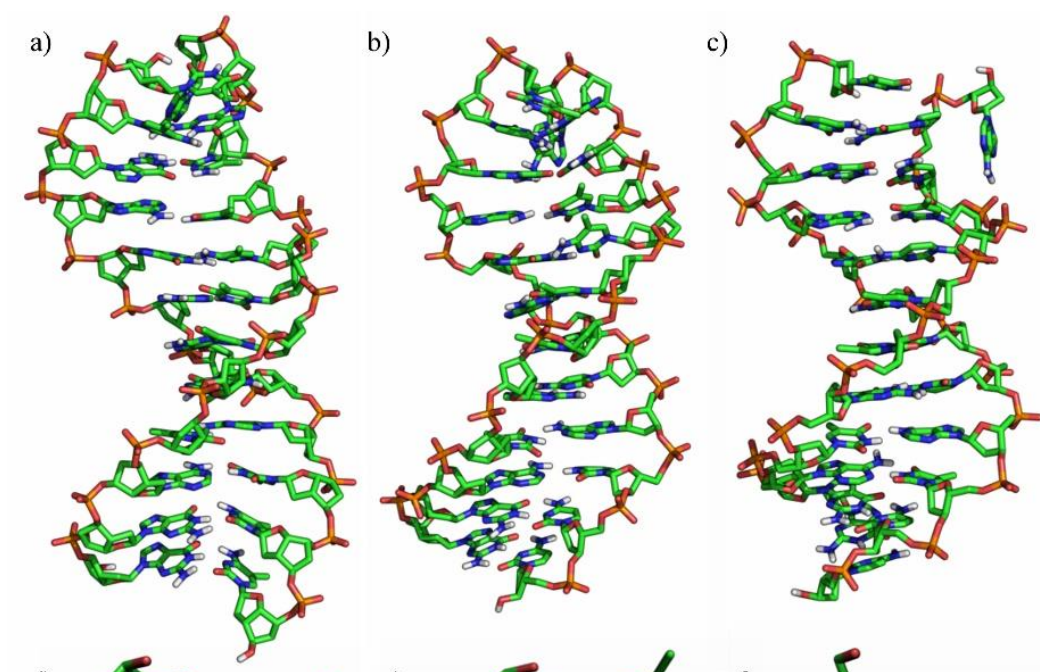

g)
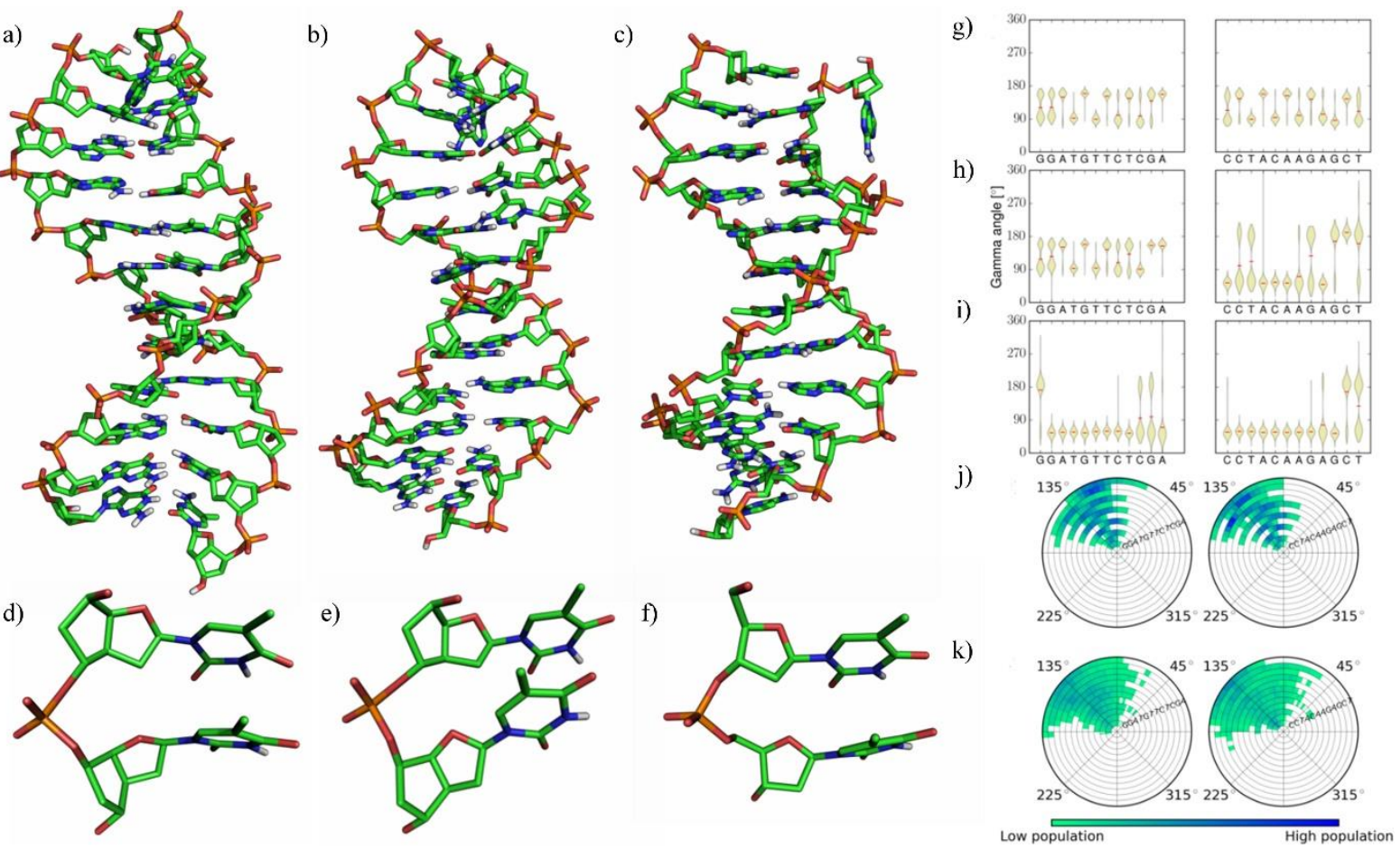

Figure 7.

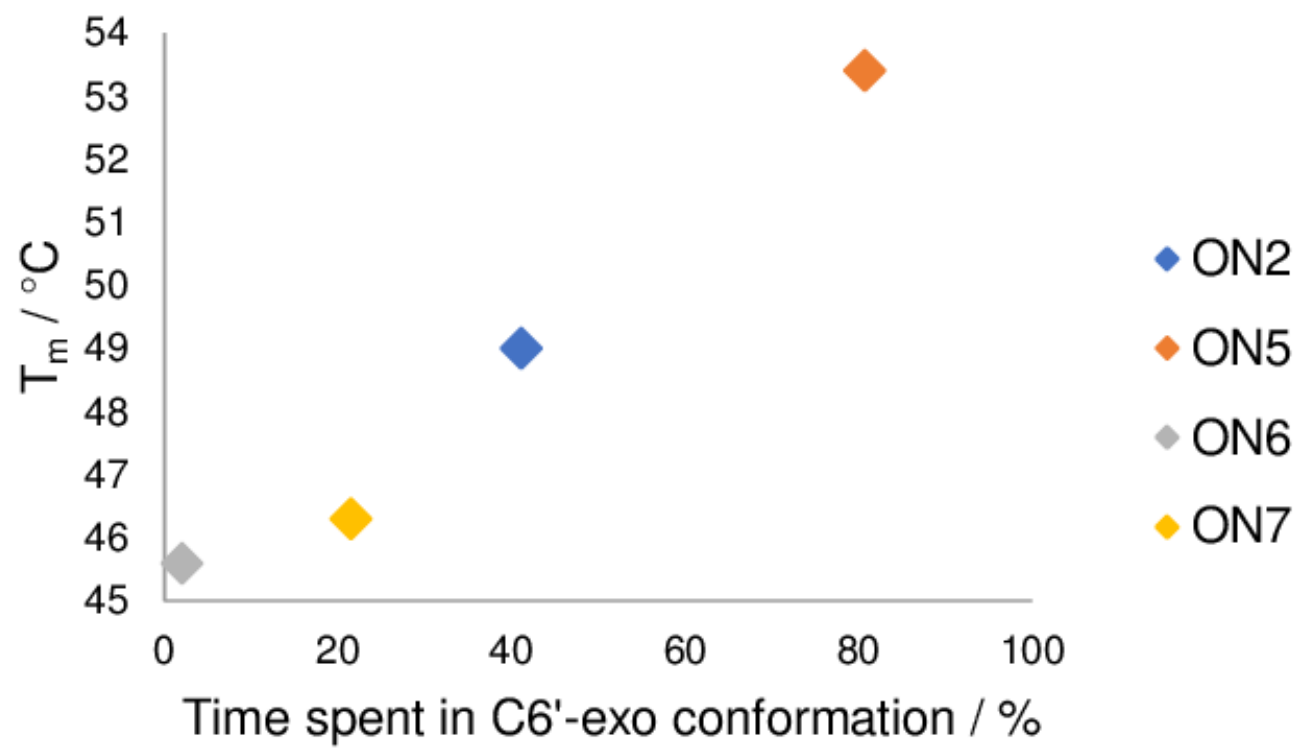

Keywords: DNA structures $•$ Molecular modelling • Oligonucleotides $•$ Synthetic Biology $•$ Xeno nucleic acids 